Supporting Information

for

\title{
Organocatalytic Synthesis of Sulfoximidoyl-Containing Carbamates from Sulfoximines and Morita-Baylis-Hillman Carbonates
}

\author{
Zhen Li, Marcus Frings, Hao Yu, and Carsten Bolm*
}

Institute of Organic Chemistry, RWTH Aachen University,

Landoltweg 1, 52056 Aachen, Germany

Table of Content

1. General information

2. Experimental section

3. Characterizing data

4. References

5. ${ }^{1} \mathrm{H}$ and ${ }^{13} \mathrm{C}$ NMR spectra 


\section{General information}

Unless otherwise stated, all reagents were purchased from commercial suppliers and used without further purification. All the solvents were treated according to general methods. All product mixtures were analyzed by thin layer chromatography using aluminum foil backed silica TLC plates with a fluorescent indicator from Merck. UV-active compounds were detected with a UV lamp $(\lambda=254 \mathrm{~nm})$. For flash column chromatography, silica gel was used as stationary phase. ${ }^{1} \mathrm{H}$ and ${ }^{13} \mathrm{C}$ NMR spectra were recorded on either a Varian V-NMRS 600, Varian V-NMRS 400 or Varian Mercury 300 in deuterated chloroform. Chemical shifts $(\delta)$ are reported in parts per million (ppm), and spin-spin coupling constants $(J)$ are given in $\mathrm{Hz}$, while multiplicities are abbreviated by br s (broad singlet), s (singlet), d (doublet), t (triplet), q (quartet) and $\mathrm{m}$ (multiplet). Almost all products were diastereomers, which could not be separated by column chromatography. In the ${ }^{1} \mathrm{H}$ NMR spectra, most of the signals of the diastereomers overlapped in the aromatic region, making it impossible to distinguish them. Consequently, the diastereomers were treated as "single" compounds in regard to ${ }^{1} \mathrm{H}$ NMR integration. The IR spectra were recorded on a PerkinElmer Spectrum 100 spectrometer with an attached UATR device Diamond KRS-5. All IR data were collected by attenuated total reflectance (ATR) and wave numbers $v$ are given in $\mathrm{cm}^{-1}$. Mass spectra were recorded with a Finnigan SSQ Finnigan 7000 spectrometer (EI, $70 \mathrm{eV}$ ). High resolution mass spectra (HRMS) were obtained on a Thermo Scientific LTQ Orbitrap XL spectrometer. Substrates 1 and $\mathbf{2}$ were prepared according to reported procedures. $^{1,2}$

\section{Experimental section}

\subsection{General procedure for the synthesis of sulfoximidoyl-containing carbamates}

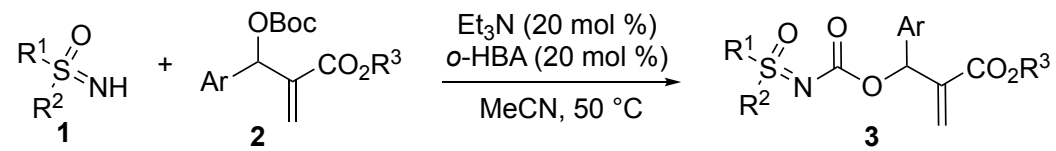

A mixture of sulfoximine $1(0.1 \mathrm{mmol})$, Morita-Baylis-Hillman carbonate 2 (3.0 equiv, $0.3 \mathrm{mmol}), \mathrm{Et}_{3} \mathrm{~N}$ $(2.8 \mu \mathrm{L}, 20 \mathrm{~mol} \%, 0.02 \mathrm{mmol})$, and ortho-hydroxybenzoic acid (2.8 mg, $20 \mathrm{~mol} \%, 0.02 \mathrm{mmol})$ in MeCN (1 $\mathrm{mL}$ ) was stirred at $50{ }^{\circ} \mathrm{C}$ under air in a sealed tube for $24 \mathrm{~h}$. Then, the solvent was removed in vacuo, and the product was purified by flash chromatography on silica gel ( $n$-pentane/ethyl acetate) to give 3 .

Scale-up experiment: A mixture of sulfoximine 1a (155.0 mg, $1.0 \mathrm{mmol})$, Morita-Baylis-Hillman carbonate 2a (876.4 mg, 3.0 equiv, $3.0 \mathrm{mmol}), \mathrm{Et}_{3} \mathrm{~N}(28 \mu \mathrm{L}, 20 \mathrm{~mol} \%, 0.2 \mathrm{mmol})$, and $o$-HBA $(28.0 \mathrm{mg}, 20 \mathrm{~mol} \%$, $0.2 \mathrm{mmol})$ in $\mathrm{MeCN}(10 \mathrm{~mL})$ was stirred at $50{ }^{\circ} \mathrm{C}$ under air in a sealed tube for $24 \mathrm{~h}$. Then, the solvent was removed in vacuo, and the product was purified by flash chromatography on silica gel ( $n$-pentane/ethyl acetate) to give product $3 \mathbf{a a}$ ( $335.7 \mathrm{mg}, 87 \%$ yield). 


\section{Characterization Data}

\section{$N$-\{[(2-(Methoxycarbonyl)-1-phenylallyl)oxy|carbonyl $\}-S$-methyl-S-phenylsulfoximine (3aa)}

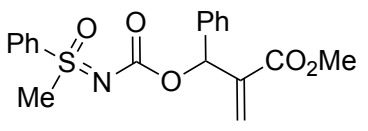

Prepared from 1a and 2a following the general procedure; white solid (35.8 $\mathrm{mg}$, $96 \%$ yield, 1.4:1 dr). $\mathrm{R}_{\mathrm{f}}=0.48$ ( $n$-pentane/EtOAc, 1:2). ${ }^{1} \mathrm{H}$ NMR $(600 \mathrm{MHz}$, $\mathrm{CDCl}_{3}$, mixture of diastereomers) $\delta 7.96-7.87(\mathrm{~m}, 2 \mathrm{H}), 7.68-7.47(\mathrm{~m}, 3 \mathrm{H})$, 7.39-7.15 (m, 5H), [6.52 (s), 6.50 (s), 1H], [6.43 (s), 6.31 (s), 1H], [5.96 (s), $5.84(\mathrm{~s}), 1 \mathrm{H}], 3.63$ (s, 3H), [3.26 (s), $3.24(\mathrm{~s}), 3 \mathrm{H}] .{ }^{13} \mathrm{C}\left\{{ }^{1} \mathrm{H}\right\}$ NMR (151 MHz, $\mathrm{CDCl}_{3}$, mixture of diastereomers) $\delta 165.42,165.41,157.2$, $156.8,139.8,139.7,138.3,138.2,138.1,137.8,134.0,133.9,129.7,128.3,128.2,128.1,128.0,127.7,127.6$, $127.34,127.28,125.7,125.6,74.93,74.88,51.8,44.7,44.6$. IR (ATR): $v=3026,2952,2103,1909,1715$, 1674, 1582, 1445, 1404, 1233, 1147, 1085, 973, 905, 847, 736, 697. MS (ESI): ([M+Na] $\left.]^{+}\right) 396.0870$. HRMS (ESI): $m / z$ calcd. for $\mathrm{C}_{19} \mathrm{H}_{19} \mathrm{NO}_{5} \mathrm{SNa}^{+}: 396.0876$, found: 396.0874 .

\section{$N$-\{[(2-(Methoxycarbonyl)-1-phenylallyl)oxy]carbonyl $\}-S$-methyl-S-(p-tolyl)sulfoximine (3ba)}

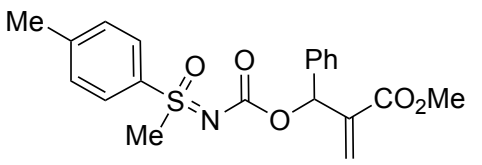

Prepared from 1b and 2a following the general procedure; white solid (34.1 mg, 88\% yield, $1.3: 1 \mathrm{dr}) . \mathrm{R}_{\mathrm{f}}=0.60(n$-pentane/EtOAc, $1: 2) .{ }^{1} \mathrm{H}$ NMR (600 MHz, $\mathrm{CDCl}_{3}$, mixture of diastereomers) $\delta[7.80$ (d, $J=8.2$ $\mathrm{Hz}) 7.77(\mathrm{~d}, J=8.2 \mathrm{~Hz}), 2 \mathrm{H}], 7.43-7.20(\mathrm{~m}, 7 \mathrm{H}),[6.53$ (s), 6.51(s), $1 \mathrm{H}],[6.36(\mathrm{~s}), 6.32(\mathrm{~s}), 1 \mathrm{H}],[5.98(\mathrm{~s}), 5.87(\mathrm{~s}), 1 \mathrm{H}],[3.65(\mathrm{~s}), 3.64(\mathrm{~s}), 3 \mathrm{H}],[3.25(\mathrm{~s}), 3.23(\mathrm{~s}), 3 \mathrm{H}],[2.44(\mathrm{~s})$, $2.43(\mathrm{~s}), 3 \mathrm{H}] .{ }^{13} \mathrm{C}\left\{{ }^{1} \mathrm{H}\right\}$ NMR (151 MHz, $\mathrm{CDCl}_{3}$, mixture of diastereomers) $\delta 165.4,157.3,156.9,145.1$, 145.0, 139.8, 139.7, 138.1, 137.9, 135.2, 135.1, 130.30, 130.29, 128.3, 128.2, 128.1, 128.0, 127.8, 127.7, 127.4, 127.3, 125.6, 125.5, 74.9, 74.8, 51.8, 44.9, 44.8, 21.6. IR (ATR): $v=2955,2106,1927,1694,1439$, 1235, 971, 731. MS (ESI): ([M+Na $\left.]^{+}\right)$410.1021. HRMS (ESI): $m / z$ calcd. for $\mathrm{C}_{20} \mathrm{H}_{21} \mathrm{O}_{5} \mathrm{NSNa}^{+}: 410.1033$, found: 410.1028 .

\section{$N$-\{[(2-(Methoxycarbonyl)-1-phenylallyl)oxy]carbonyl $\}-S$-methyl-S-(4-fluorophenyl)sulfoximine (3ca)}

$\mathrm{F}$<smiles>C=C(C(C)=O)C(OC(=O)N=S(C)(=O)c1ccc(C)cc1)c1ccccc1</smiles>
Prepared from 1c and 2a following the general procedure; colorless oil (36.0 mg, 92\% yield, 1.3:1 dr). $\mathrm{R}_{\mathrm{f}}=0.54$ (n-pentane/EtOAc, $\left.1: 2\right) .{ }^{1} \mathrm{H}$ $\operatorname{NMR}\left(600 \mathrm{MHz}, \mathrm{CDCl}_{3}\right.$, mixture of diastereomers) $\delta 7.98-7.86(\mathrm{~m}, 2 \mathrm{H})$ $7.42-7.14(\mathrm{~m}, 7 \mathrm{H}),[6.52(\mathrm{~s}), 6.51(\mathrm{~s}), 1 \mathrm{H}],[6.36(\mathrm{~s}), 6.35(\mathrm{~s}), 1 \mathrm{H}],[5.96$ (s), 5.89 (s), 1H], [3.66 (s), 3.65 (s), 3H], [3.27 (s), $3.26(\mathrm{~s}), 3 \mathrm{H}] .{ }^{13} \mathrm{C}\left\{{ }^{1} \mathrm{H}\right\}$ NMR $\left(151 \mathrm{MHz}, \mathrm{CDCl}_{3}\right.$, mixture of diastereomers) $\delta 165.93\left(\mathrm{~d}, J_{C-F}=257.3 \mathrm{~Hz}\right), 165.89\left(\mathrm{~d}, J_{C-F}=257.1 \mathrm{~Hz}\right), 165.4,157.2,156.6,139.7$, $139.6,138.0$, 137.7, 134.2, 134.0, 130.33, 130.27, 130.23, 130.16, 128.3, 128.24, 128.18, 128.1, 127.7, 127.6, 125.8, 125.6, $117.06\left(\mathrm{~d}, J_{C-F}=22.9 \mathrm{~Hz}\right), 117.03\left(\mathrm{~d}, J_{C-F}=22.9 \mathrm{~Hz}\right), 75.03,74.99,51.9,44.9,44.8$. IR $(\mathrm{ATR}): v=3024,2943,2254,2101,1915,1713,1677,1589,1493,1442,1402,1232,1145,1088,976,904$ 835, 776, 709. MS (ESI): ([M+Na $\left.]^{+}\right)$414.0761. HRMS (ESI): $m / z$ calcd. for $\mathrm{C}_{19} \mathrm{H}_{18} \mathrm{O}_{5} \mathrm{NFSNa}^{+}: 414.0782$, found: 414.0779 .

\section{$N$-\{[(2-(Methoxycarbonyl)-1-phenylallyl)oxy]carbonyl $\}-S$-methyl-S-(4-chlorophenyl)sulfoximine (3da)}

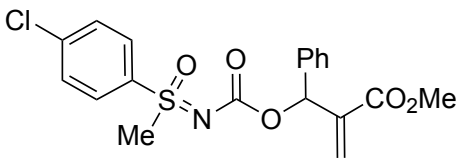
Prepared from 1d and 2a following the general procedure; white solid (33.8 mg, 83\% yield, 1.3:1 dr). $\mathrm{R}_{\mathrm{f}}=0.69$ (n-pentane/EtOAc, $\left.1: 2\right) .{ }^{1} \mathrm{H}$ NMR (600 MHz, $\mathrm{CDCl}_{3}$, mixture of diastereomers) $\delta[7.85$ (d, $J=8.6$ $\mathrm{Hz}), 7.81(\mathrm{~d}, J=8.6 \mathrm{~Hz}), 2 \mathrm{H}],[7.49-7.45(\mathrm{~m}), 7.55-7.50(\mathrm{~m}), 2 \mathrm{H}]$, 7.39-7.15 (m, 5H), [6.52 (s), $6.50(\mathrm{~s}), 1 \mathrm{H}],[6.36(\mathrm{~s}), 6.34$ (s) 1H], [5.96 (d, J=0.7 Hz), $5.88(\mathrm{~d}, J=0.7 \mathrm{~Hz})$, $1 \mathrm{H}],[3.66(\mathrm{~s}), 3.65(\mathrm{~s}), 3 \mathrm{H}],[3.26(\mathrm{~d}, J=1.1 \mathrm{~Hz}), 3.24(\mathrm{~d}, J=1.1 \mathrm{~Hz}), 3 \mathrm{H}] .{ }^{13} \mathrm{C}\left\{{ }^{1} \mathrm{H}\right\} \mathrm{NMR}(151 \mathrm{MHz}$, 
$\mathrm{CDCl}_{3}$, mixture of diastereomers) $\delta 165.37,165.35,157.1,156.5,140.85,140.78,139.7,139.5,137.9,137.6$, $136.8,136.6,130.00,129.98,128.9,128.8,128.3,128.25,128.20,128.1,127.7,127.6,125.8,125.6,75.1$, 75.0, 51.89, 51.87, 44.8, 44.6. IR (ATR): $v=3024,2096,1697,1439,1237,969,734 . \mathrm{MS}(\mathrm{ESI}):\left([\mathrm{M}+\mathrm{Na}]^{+}\right)$ 430.0472. HRMS (ESI): $\mathrm{m} / z$ calcd. for $\mathrm{C}_{19} \mathrm{H}_{18} \mathrm{O}_{5} \mathrm{NClSNa}^{+}$: 430.0486 , found: 430.0474 .

$N$-\{[(2-(Methoxycarbonyl)-1-phenylallyl)oxy]carbonyl $\}-S$-methyl-S-(4-acetylphenyl) sulfoximine (3ea)

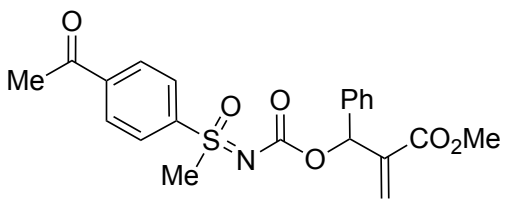

Prepared from 1e and 2a following the general procedure; white solid (20.3 mg, 49\% yield, 1.3:1 dr). $\mathrm{R}_{\mathrm{f}}=0.44$ (n-pentane/EtOAc, $1: 2)$. ${ }^{1} \mathrm{H}$ NMR (600 MHz, $\mathrm{CDCl}_{3}$, mixture of diastereomers) $\delta 8.13-$ $7.92(\mathrm{~m}, 4 \mathrm{H}), 7.41-7.13(\mathrm{~m}, 5 \mathrm{H}),[6.51(\mathrm{~s}), 6.49(\mathrm{~s}), 1 \mathrm{H}],[6.36(\mathrm{~s})$, 6.34 (s), 1H], [5.96 (s), 5.88 (s), 1H], [3.65 (s), 3.64 (s), 3H], [3.29 (s), 3.27 (s), 3H], [2.65 (s), 2.64 (s), 3H]. ${ }^{13} \mathrm{C}\left\{{ }^{1} \mathrm{H}\right\}$ NMR (151 MHz, $\mathrm{CDCl}_{3}$, mixture of diastereomers) $\delta 196.4,196.3,165.4,165.3,157.2,156.5$, $142.3,142.2$, 141.04, 140.95, 139.6, 139.5, 137.9, 137.6, 129.32, 129.30, 128.3, 128.2, 128.1, 127.8, 127.73, 127.71, 127.6, 125.8, 125.6, 75.14, 75.10, 51.9, 44.4, 44.3, 26.91, 26.89. IR (ATR): $v=3021,2934,2254$, $2100,1684,1496,1441,1399,1358,1236,1142,1084,972,903,840,782,707 . \mathrm{MS}(\mathrm{ESI}):\left([\mathrm{M}+\mathrm{Na}]^{+}\right)$ 438.0975. HRMS (ESI): $m / z$ calcd. for $\mathrm{C}_{21} \mathrm{H}_{21} \mathrm{O}_{6} \mathrm{NSNa}^{+}$: 438.0982, found: 438.0983 .

\section{$N$-\{[(2-(Methoxycarbonyl)-1-phenylallyl)oxy]carbonyl $\}-S$-methyl-S-(3-bromophenyl)sulfoximine (3fa)}

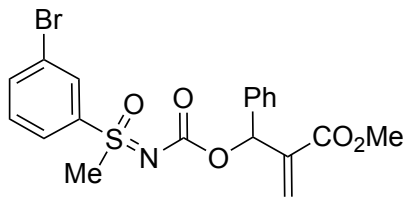

Prepared from 1f and 2a following the general procedure; light yellow solid (34.7 mg, 77\% yield, 1.4:1 dr). $\mathrm{R}_{\mathrm{f}}=0.56$ ( $n$-pentane/EtOAc, $\left.1: 2\right) .{ }^{1} \mathrm{H}$ NMR (600 MHz, $\mathrm{CDCl}_{3}$, mixture of diastereomers) $\delta[8.08(\mathrm{t}, J=1.8 \mathrm{~Hz}$ ), $8.04(\mathrm{t}, J=1.8 \mathrm{~Hz}), 1 \mathrm{H}], 7.88-7.72(\mathrm{~m}, 2 \mathrm{H}), 7.45-7.35(\mathrm{~m}, 2 \mathrm{H}), 7.32-7.18$

$(\mathrm{m}, 4 \mathrm{H}),[6.52(\mathrm{~s}), 6.50(\mathrm{~s}), 1 \mathrm{H}],[6.37$ (s), $6.35(\mathrm{~s}), 1 \mathrm{H}],[5.95(\mathrm{~s}), 5.87(\mathrm{~s}), 1 \mathrm{H}]$, [3.66 (s), $3.65(\mathrm{~s}), 3 \mathrm{H}]$, [3.27 (s), $3.25(\mathrm{~s}), 3 \mathrm{H}] .{ }^{13} \mathrm{C}\left\{{ }^{1} \mathrm{H}\right\} \mathrm{NMR}\left(151 \mathrm{MHz}, \mathrm{CDCl}_{3}\right.$, mixture of diastereomers) $\delta 165.4,157.1,156.6$, $140.3,140.1$, 139.7, 139.6, 137.9, 137.6, 137.1, 137.0, 131.1, 130.3, 130.2, 128.4, 128.3, 128.21, 128.18, $127.65,127.58,125.9,125.8,125.7,123.7,75.2,75.1,51.9,44.7,44.5$. IR (ATR): $v=3066,3024,2929$, 2109, 1715, 1674, 1571, 1495, 1455, 1405, 1233, 1145, 1104, 974, 906, 847, 778, 728, 702, 675. MS (ESI): $\left([\mathrm{M}+\mathrm{Na}]^{+}\right)$475.9965. HRMS (ESI): $m / z$ calcd. for $\mathrm{C}_{19} \mathrm{H}_{18} \mathrm{O}_{5} \mathrm{NBrSNa}^{+}: 473.9981$, found: 473.9985 .

\section{$N$-\{[(2-(Methoxycarbonyl)-1-phenylallyl)oxy]carbonyl $\}-S$-methyl-S-(2-methoxyphenyl)sulfoximine}

(3ga)<smiles>C=C(C(C)=O)C(OC(=O)N=S(C)(=O)c1ccccc1OC)c1ccccc1</smiles>

Prepared from 1g and 2a following the general procedure; colorless oil (31.4 mg, 78\% yield, 1.3:1 dr). $\mathrm{R}_{\mathrm{f}}=0.33$ (n-pentane/EtOAc, $\left.1: 2\right) .{ }^{1} \mathrm{H}$ NMR (600 $\mathrm{MHz}, \mathrm{CDCl}_{3}$, mixture of diastereomers) $\delta 8.03-7.98(\mathrm{~m}, 1 \mathrm{H}), 7.63-$ $7.56(\mathrm{~m}, 1 \mathrm{H}),[7.36(\mathrm{~s}), 7.34(\mathrm{~s}), 1 \mathrm{H}], 7.29-7.18(\mathrm{~m}, 4 \mathrm{H}), 7.15-7.07(\mathrm{~m}$, 1H), 7.03-6.96 (m, 1H), [6.50 (s), $6.49(\mathrm{~s}), 1 \mathrm{H}], 6.32(\mathrm{~s}, 1 \mathrm{H})$, [5.94 (s), $5.88(\mathrm{~s}), 1 \mathrm{H}],[3.86(\mathrm{~s}), 3.82(\mathrm{~s}), 3 \mathrm{H}]$, [3.64 (s), 3,62 (s), 3H], [3.40 (s), $3.38(\mathrm{~s}), 3 \mathrm{H}] .{ }^{13} \mathrm{C}\left\{{ }^{1} \mathrm{H}\right\}$ NMR (151 MHz, $\mathrm{CDCl}_{3}$, mixture of diastereomers) $\delta 165.5,157.0,156.6,156.45,156.42,139.9,138.3,138.0,135.9,135.8,131.02,130.98,128.2,128.0,127.9$, $127.81,127.79,125.5,125.3,121.10,121.07,112.6,112.5,74.6,74.4,56.31,56.29,51.79,51.77,42.8,42.7$. IR (ATR): $v=3619,3021,2948,2251,2089,1926,1681,1588,1468,1240,1140,1070,979,902,846,726$. HRMS (ESI): $m / z$ calcd. for $\mathrm{C}_{20} \mathrm{H}_{21} \mathrm{O}_{6} \mathrm{NSNa}^{+}:$426.0982, found: 426.0981 . 


\section{$N$-\{[(2-(Methoxycarbonyl)-1-phenylallyl)oxy]carbonyl $\}-S$-methyl-S-(pyridin-2-yl)sulfoximine (3ha)}

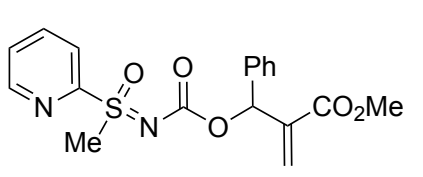

Prepared from $\mathbf{1 h}$ and $\mathbf{2 a}$ following the general procedure; colorless oil (35.2 mg, 94\% yield, 1.3:1 dr). $\mathrm{R}_{\mathrm{f}}=0.38$ (n-pentane/EtOAc, 1:2). ${ }^{1} \mathrm{H}$ NMR $\left(600 \mathrm{MHz}, \mathrm{CDCl}_{3}\right.$, mixture of diastereomers) $\delta 8.70-8.62(\mathrm{~m}, 1 \mathrm{H}),[8.21(\mathrm{~d}$, $J=8.0 \mathrm{~Hz}), 8.15(\mathrm{~d}, J=8.0 \mathrm{~Hz}), 1 \mathrm{H}],[7.95(\mathrm{td}, J=7.8,1.6 \mathrm{~Hz}), 7.87(\mathrm{td}, J$ $=7.8,1.6 \mathrm{~Hz}), 1 \mathrm{H}],[7.56-7.52(\mathrm{~m}), 7.52-7.48(\mathrm{~m}), 1 \mathrm{H}], 7.38-7.32(\mathrm{~m}, 1 \mathrm{H}), 7.31-7.21(\mathrm{~m}, 4 \mathrm{H}),[6.47(\mathrm{~s})$, $6.45(\mathrm{~s}), 1 \mathrm{H}],[6.35(\mathrm{~s}), 6.34(\mathrm{~s}), 1 \mathrm{H}],[5.97(\mathrm{~s}), 5.91(\mathrm{~s}), 1 \mathrm{H}],[3.64(\mathrm{~s}), 3.62(\mathrm{~s}), 3 \mathrm{H}],[3.42(\mathrm{~s}), 3.40(\mathrm{~s}), 3 \mathrm{H}]$. ${ }^{13} \mathrm{C}\left\{{ }^{1} \mathrm{H}\right\}$ NMR (151 MHz, $\mathrm{CDCl}_{3}$, mixture of diastereomers) $\delta 165.4,157.3,156.9,156.20,156.16,150.01$, $149.99,139.7,139.5,138.2,138.1,137.83,137.76,128.3,128.2,128.14,128.11,127.8,127.7,127.5,127.4$, $125.7,125.6,123.42,123.41,75.0,74.9,51.85,51.83,40.1,39.9$. IR (ATR): $v=3025,2944,2252,2101$, 1916, 1714, 1673, 1578, 1494, 1438, 1237, 1143, 1081, 977, 904, 848, 765, 723. MS (ESI): ([M+Na $\left.]^{+}\right)$ 397.0822. HRMS (ESI): $m / z$ calcd. for $\mathrm{C}_{18} \mathrm{H}_{18} \mathrm{O}_{5} \mathrm{~N}_{2} \mathrm{SNa}^{+}: 397.0829$, found: 397.0826 .

\section{$N$-\{[(2-(Methoxycarbonyl)-1-phenylallyl)oxy]carbonyl $\}-S$-methyl-S-styrylsulfoximine (3ia)}<smiles>C=C(C(C)=O)C(=O)OC(C(=O)NS(C)(=O)=Cc1ccccc1)c1ccccc1</smiles>

Prepared from 1i and 2a following the general procedure; colorless oil (32.3 mg, 81\% yield, 1.3:1 dr). $\mathrm{R}_{\mathrm{f}}=0.58$ (n-pentane/EtOAc, $\left.1: 2\right) .{ }^{1} \mathrm{H}$ NMR (600 MHz, $\mathrm{CDCl}_{3}$, mixture of diastereomers) $\delta$ 7.72-7.62 (m, 1H), 7.52-7.34 (m, 7H), 7.32-7.19 (m, 3H), [6.96 (d, $J=15.3 \mathrm{~Hz}), 6.91$ (d, $J=15.3 \mathrm{~Hz}), 1 \mathrm{H}], 6.59$ (s, 1H), [6.38 (s), 6.37 (s), 1H], [6.02 (s), 5.99 (s), 1H], [3.67 (s), $3.66(\mathrm{~s}), 3 \mathrm{H}],[3.291(\mathrm{~s}), 3.287(\mathrm{~s}), 3 \mathrm{H}] .{ }^{13} \mathrm{C}\left\{{ }^{1} \mathrm{H}\right\}$ NMR $\left(151 \mathrm{MHz}, \mathrm{CDCl}_{3}\right.$, mixture of diastereomers) $\delta 165.5,157.5,157.3,145.49,145.47,139.9,139.8,138.05$, $138.02,131.8,131.7,129.19,129.17,128.79,128.77,128.34,128.28,128.2,128.1,127.8,127.7,125.72$, 125.68, 123.6, 123.5, 74.90, 74.86, 51.9, 42.9, 42.8, 28.2. IR (ATR): $v=3031,2944,2252,2091,1711,1672$, 1496, 1444, 1236, 1146, 976, 903, 826, 728. MS (ESI): ([M+Na $\left.]^{+}\right) 422.1043$. HRMS (ESI): $\mathrm{m} / z$ calcd. for $\mathrm{C}_{21} \mathrm{H}_{21} \mathrm{O}_{5} \mathrm{NSNa}^{+}:$422.1033, found: 422.1034 .

\section{$N$-\{[(2-(Methoxycarbonyl)-1-phenylallyl)oxy]carbonyl $\}-S$-methyl-S-(tert-butyl)sulfoximine (3ja)}<smiles>C=C(C(=O)OC(=O)N=S(C)(=O)C(C)(C)C)c1ccccc1</smiles>

Prepared from $\mathbf{1 j}$ and $\mathbf{2 a}$ following the general procedure; colorless oil (12.0 $\mathrm{mg}, 34 \%$ yield, $1.3: 1 \mathrm{dr}) . \mathrm{R}_{\mathrm{f}}=0.44$ (n-pentane/EtOAc, 1:2). ${ }^{1} \mathrm{H}$ NMR $(600$ $\mathrm{MHz}, \mathrm{CDCl}_{3}$, mixture of diastereomers) $\delta$ 7.44-7.40 (m, 2H), 7.35-7.30 (m, 2H), 7.29-7.26 (m, 1H), [6.60 (s), $6.58(\mathrm{~s}), 1 \mathrm{H}],[6.40(\mathrm{~s}), 6.39(\mathrm{~s}), 1 \mathrm{H}],[6.03(\mathrm{~s}), 5.98(\mathrm{~s}), 1 \mathrm{H}],[3.694(\mathrm{~s})$, 3.687 (s), 3H], [3.22 (s), 3.17 (s), 3H], [1.48(s), 1.47 (s), 9H]. ${ }^{13} \mathrm{C}\left\{{ }^{1} \mathrm{H}\right\} \mathrm{NMR}\left(151 \mathrm{MHz}, \mathrm{CDCl}_{3}\right.$, mixture of diastereomers) $\delta 165.7,165.6,158.7,158.1,140.1,140.0,138.3,138.2,128.4,128.3,128.1,128.0,127.8$, 127.7, 126.0, 125.7, 74.94, 74.88, 60.6, 51.9, 32.5, 32.3, 23.05, 23.00. IR (ATR): $v=3618,2948,2102,1716$, 1666, 1449, 1247, 1151, 988, 900, 851, 770, 705. MS (ESI): ([M+Na] $\left.]^{+}\right) 376.1177$. HRMS (ESI): $m / z$ calcd. for $\mathrm{C}_{17} \mathrm{H}_{23} \mathrm{O}_{5} \mathrm{NSNa}^{+}$: 376.1189 , found: 376.1192 .

\section{$N$-\{[(2-(Methoxycarbonyl)-1-phenylallyl)oxy]carbonyl\}-tetrahydrothiophenesulfoximine (3ka)}

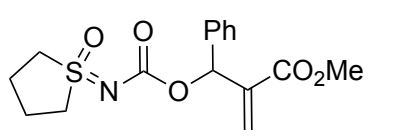

Prepared from 1k and 2a following the general procedure; colorless oil (22.2 $\mathrm{mg}, 66 \%$ yield). $\mathrm{R}_{\mathrm{f}}=0.25$ (n-pentane/EtOAc, 1:2). ${ }^{1} \mathrm{H}$ NMR $(600 \mathrm{MHz}$,

$\left.\mathrm{CDCl}_{3}\right) \delta 7.44-7.38(\mathrm{~m}, 2 \mathrm{H}), 7.35-7.25(\mathrm{~m}, 3 \mathrm{H}), 6.59(\mathrm{~s}, 1 \mathrm{H}), 6.39(\mathrm{~s}, 1 \mathrm{H})$, 5.99 (s, 1H), 3.69 (s, 3H), 3.62-3.54 (m, 2H), 3.29-3.21 (m, 2H), 2.39-2.16 (m, 4H). ${ }^{13} \mathrm{C}\left\{{ }^{1} \mathrm{H}\right\}$ NMR $(151$ $\left.\mathrm{MHz}, \mathrm{CDCl}_{3}\right) \delta 165.5,158.6,139.8,138.0,128.4,128.2,127.7,125.8,75.0,52.8,52.7,51.9,28.2,23.5$. IR (ATR): $v=3614,2954,2326,2095,1917,1716,1661,1444,1241,1149,1083,986,907,850,771,711$. MS (ESI): ([M+Na] $]^{+}$) 360.0875. HRMS (ESI): $\mathrm{m} / z$ calcd. for $\mathrm{C}_{16} \mathrm{H}_{19} \mathrm{O}_{5} \mathrm{NSNa}^{+}: 360.0876$, found: 360.0875 . 


\section{$N$-\{[(2-(Methoxycarbonyl)-1-phenylallyl)oxy]carbonyl $\}-S, S$-diphenylsulfoximine (3la)}

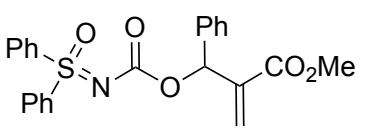

Prepared from 11 and 2a following the general procedure; white solid (23.5 mg, $54 \%$ yield). $\mathrm{R}_{\mathrm{f}}=0.77$ ( $n$-pentane/EtOAc, 1:2). ${ }^{1} \mathrm{H}$ NMR (600 MHz, $\left.\mathrm{CDCl}_{3}\right) \delta$

8.00-7.91 (m, 4H), 7.58-7.43 (m, 6H), 7.24-7.20 (m, 5H), 6.54 (s, 1H), 6.33 (s, 1H), $5.92(\mathrm{~s}, 1 \mathrm{H}), 3.63(\mathrm{~s}, 3 \mathrm{H}) .{ }^{13} \mathrm{C}\left\{{ }^{1} \mathrm{H}\right\}$ NMR $\left(151 \mathrm{MHz}, \mathrm{CDCl}_{3}\right) \delta 165.4,156.7,139.7,139.6,139.4,138.0$, $133.45,133.36,129.49,129.48,128.2,128.0,127.7,127.6,125.6,75.08,75.06,51.85,51.82$. IR (ATR): $v=$ 3057, 2952, 2323, 2087, 1696, 1447, 1228, 982, 904, 700. MS (ESI): ([M+Na $\left.]^{+}\right)$458.1026. HRMS (ESI): $m / z$ calcd. for $\mathrm{C}_{24} \mathrm{H}_{21} \mathrm{O}_{5} \mathrm{NSNa}^{+}: 458.1033$, found: 458.1028 .

\section{$N$-\{[(2-(Methoxycarbonyl)-1-(p-tolyl)allyl)oxy]carbonyl $\}-S$-methyl-S-phenylsulfoximine (3ab)}

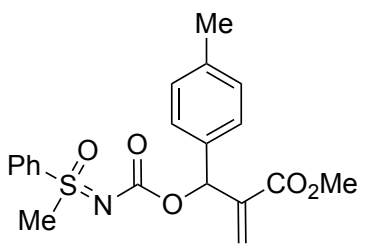

Prepared from 1a and 2b following the general procedure; white solid (34.8 $\mathrm{mg}, 90 \%$ yield, 1.4:1 dr). $\mathrm{R}_{\mathrm{f}}=0.62$ (n-pentane/EtOAc, $\left.1: 2\right) .{ }^{1} \mathrm{H}$ NMR $(600$ $\mathrm{MHz}, \mathrm{CDCl}_{3}$, mixture of diastereomers) $\delta[7.93(\mathrm{~d}, J=8.1 \mathrm{~Hz}), 7.91(\mathrm{~d}, J=8.1$ $\mathrm{Hz}), 2 \mathrm{H}], 7.72-7.49(\mathrm{~m}, 3 \mathrm{H}), 7.31-7.23(\mathrm{~m}, 1 \mathrm{H}), 7.16-6.99(\mathrm{~m}, 2 \mathrm{H}),[6.50(\mathrm{~s})$, $6.48(\mathrm{~s}), 1 \mathrm{H}],[6.33(\mathrm{~s}), 6.31(\mathrm{~s}), 1 \mathrm{H}],[5.96(\mathrm{~s}), 5.85(\mathrm{~s}), 1 \mathrm{H}], 3.64(\mathrm{~s}, 3 \mathrm{H})$, [3.27 (s), $3.26(\mathrm{~s}), 3 \mathrm{H}],[2.31(\mathrm{~s}), 2.29(\mathrm{~s}), 3 \mathrm{H}] .{ }^{13} \mathrm{C}\left\{{ }^{1} \mathrm{H}\right\}$ NMR (151 MHz, $\mathrm{CDCl}_{3}$, mixture of diastereomers) $\delta 165.49,165.46,157.2,157.0,139.9,139.8,138.4,138.3,137.9,137.8,135.1,134.9,133.9,133.8,129.6$, 129.0, 128.9, 127.7, 127.6, 127.4, 127.3, 125.4, 125.3, 74.9, 74.8, 51.8, 44.7, 44.6, 21.16, 21.15. IR (ATR): $v$ $=3019,2936,2100,1914,1713,1676,1514,1443,1235,1141,1086,973,901,853,812,738,685$. MS (ESI): ([M+Na] $]^{+}$410.1034. HRMS (ESI): $\mathrm{m} / z$ calcd. for $\mathrm{C}_{20} \mathrm{H}_{21} \mathrm{O}_{5} \mathrm{NSNa}^{+}: 410.1033$, found: 410.1032 .

\section{$N$-\{[(2-(Methoxycarbonyl)-1-(m-tolyl)allyl)oxy]carbonyl $\}-S$-methyl-S-phenylsulfoximine (3ac)}

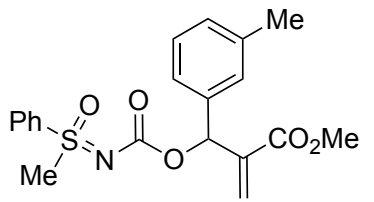

Prepared from 1a and 2c following the general procedure; white solid (31.0 mg, $80 \%$ yield, $1.3: 1 \mathrm{dr}) . \mathrm{R}_{\mathrm{f}}=0.42$ ( $n$-pentane/EtOAc, $\left.1: 2\right) .{ }^{1} \mathrm{H}$ NMR $(600 \mathrm{MHz}$, $\mathrm{CDCl}_{3}$, mixture of diastereomers) $\delta[7.93(\mathrm{~d}, J=8.2 \mathrm{~Hz}), 7.91(\mathrm{~d}, J=8.2 \mathrm{~Hz})$, $2 \mathrm{H}],[7.68-7.61(\mathrm{~m}), 7.59-7.51(\mathrm{~m}), 3 \mathrm{H}),[7.21(\mathrm{t}, J=7.8 \mathrm{~Hz}), 7.14(\mathrm{t}, J=$ $7.8 \mathrm{~Hz}), 1 \mathrm{H}], 6.98-6.90(\mathrm{~m}, 1 \mathrm{H}), 6.84-6.75(\mathrm{~m}, 2 \mathrm{H})$, [6.51 (s), $6.49(\mathrm{~s}), 1 \mathrm{H}]$, [6.35 (s), $6.32(\mathrm{~s}), 1 \mathrm{H}],[5.95$ (s), $5.82(\mathrm{~s}), 1 \mathrm{H}],[3.77(\mathrm{~d}, J=0.6 \mathrm{~Hz}), 3.75(\mathrm{~d}, J=0.6 \mathrm{~Hz}), 3 \mathrm{H}], 3.66(\mathrm{~s}, 3 \mathrm{H}),[3.27(\mathrm{~s}), 3.26(\mathrm{~s}), 3 \mathrm{H}]$. ${ }^{13} \mathrm{C}\left\{{ }^{1} \mathrm{H}\right\}$ NMR (151 MHz, $\mathrm{CDCl}_{3}$, mixture of diastereomers) $\delta 165.4,159.5,159.4,157.2,156.9,139.7$, 139.6, 139.3, 138.3, 134.0, 133.9, 129.7, 129.6, 129.3, 129.2, 127.4, 127.3, 126.0, 125.8, 120.0, 119.9, 113.9, 113.8, 113.2, 113.0, 74.8, 74.7, 55.21, 55.17, 51.9, 44.7, 44.6. IR (ATR): $v=2949,2099,1696,1450,1236$, 973, 881, 729. MS (ESI): $\left([\mathrm{M}+\mathrm{Na}]^{+}\right)$410.1036. HRMS (ESI): $\mathrm{m} / z$ calcd. for $\mathrm{C}_{20} \mathrm{H}_{21} \mathrm{O}_{5} \mathrm{NSNa}^{+}: 410.1033$, found: 410.1030 .

\section{$N$-\{[(2-(Methoxycarbonyl)-1-[4-(methylthio)phenyl]allyl)oxy]carbonyl $\}-S$-methyl-S-phenylsulfoximine} (3ad)

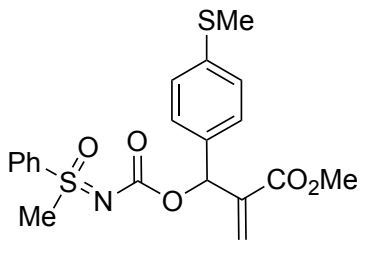

Prepared from 1a and 2d following the general procedure; white solid (31.0 $\mathrm{mg}, 74 \%$ yield, 1.3:1 dr). $\mathrm{R}_{\mathrm{f}}=0.43$ (n-pentane/EtOAc, 1:2). ${ }^{1} \mathrm{H}$ NMR (600 $\mathrm{MHz}, \mathrm{CDCl}_{3}$, mixture of diastereomers) $\delta 77.96-7.87(\mathrm{~m}, 2 \mathrm{H}), 7.70-7.62(\mathrm{~m}$, 1H), 7.59-7.51 (m, 2H), 7.29 (d, $J=8.3 \mathrm{~Hz}, 1 \mathrm{H}), 7.20-7.06$ (m, 3H), [6.48 (s), $6.46(\mathrm{~s}), 1 \mathrm{H}],[6.34(\mathrm{~s}), 6.31(\mathrm{~s}), 1 \mathrm{H}],[5.98(\mathrm{~s}), 5.85(\mathrm{~s}), 1 \mathrm{H}]$, [3.65 (s), $3.64(\mathrm{~s})$, $3 \mathrm{H}],[3.27(\mathrm{~s}), 3.26(\mathrm{~s}), 3 \mathrm{H}],[2.45(\mathrm{~s}), 2.44(\mathrm{~s}), 3 \mathrm{H}] .{ }^{13} \mathrm{C}\left\{{ }^{1} \mathrm{H}\right\} \quad \mathrm{NMR}\left(151 \mathrm{MHz}, \mathrm{CDCl}_{3}\right.$, mixture of diastereomers) $\delta 165.4,157.2,156.8,139.6,139.5,138.5,138.40,138.35,138.2,134.9,134.7,134.0,133.90$ 
129.7, 128.2, 127.34, 127.28, 126.3, 126.2, 125.5, 125.4, 74.59, 74.57, 51.9, 44.8, 44.6, 15.7. IR (ATR): $v=$ 3036, 2927, 2654, 2217, 2105, 1985, 1906, 1719, 1665, 1597, 1494, 1440, 1407, 1239, 1154, 1085, 977, 908, 847, 816, 789, 743, 685. MS (ESI): ([M+Na] $]^{+}$442.0757. HRMS (ESI): $m / z$ calcd. for $\mathrm{C}_{20} \mathrm{H}_{21} \mathrm{O}_{5} \mathrm{NS}_{2} \mathrm{Na}^{+}$: 442.0753, found: 442.0753 .

$N$-\{[(2-(Methoxycarbonyl)-1-(4-bromophenyl)allyl)oxy]carbonyl $\}-S$-methyl-S-phenylsulfoximine (3ae)<smiles>C=C(OC)C(OC(=O)N=S(C)(=O)c1ccccc1)c1ccc(Br)cc1</smiles>
Prepared from 1a and 2e following the general procedure; white solid (31.6 mg, $70 \%$ yield, 1.3:1 dr). $\mathrm{R}_{\mathrm{f}}=0.62$ ( $n$-pentane/EtOAc, 1:2). ${ }^{1} \mathrm{H}$ NMR $(600 \mathrm{MHz}$, $\mathrm{CDCl}_{3}$, mixture of diastereomers) $\delta 7.95-7.88(\mathrm{~m}, 2 \mathrm{H}), 7.70-7.52(\mathrm{~m}, 3 \mathrm{H})$, 7.44-7.24 (m, 3H), 7.07 (d, $J=8.4 \mathrm{~Hz}, 1 \mathrm{H}),[6.47$ (s), 6.45 (s), 1H], [6.36 (s), $6.32(\mathrm{~s}), 1 \mathrm{H}],[6.00(\mathrm{~s}), 5.87(\mathrm{~s}), 1 \mathrm{H}],[3.651(\mathrm{~s}), 3.647(\mathrm{~s}), 3 \mathrm{H}],[3.28(\mathrm{~s}), 3.26$

(s), 3H]. ${ }^{13} \mathrm{C}\left\{{ }^{1} \mathrm{H}\right\}$ NMR (151 MHz, $\mathrm{CDCl}_{3}$, mixture of diastereomers) $\delta 165.2,157.1,156.6,139.3,139.2$, $138.3,138.1,137.3,137.0,134.05,133.97,131.44,131.37,129.7,129.4,127.32,127.26,125.83,125.75$, $122.2,122.1,74.24,74.22,51.92,51.91,44.8,44.6$. IR (ATR): $v=3018,2940,2325,2105,1912,1711$, 1483, 1443, 1235, 1146, 1077, 974, 900, 817, 738, 684. MS (ESI): ([M+Na] $\left.{ }^{+}\right)$475.9957. HRMS (ESI): $\mathrm{m} / z$ calcd. for $\mathrm{C}_{19} \mathrm{H}_{18} \mathrm{O}_{5} \mathrm{NBrSNa}^{+}$: 473.9981, found: 473.9982 .

\section{$N$-\{[(2-(Methoxycarbonyl)-1-(3-bromophenyl)allyl)oxy]carbonyl $\}-S$-methyl-S-phenylsulfoximine (3af)}<smiles>C=C(C(C)=O)C(OC(=O)N=S(C)(=O)c1ccccc1)c1cccc(Br)c1</smiles>

Prepared from 1a and $\mathbf{2 f}$ following the general procedure; white solid (34.7 $\mathrm{mg}$, $77 \%$ yield, $1.3: 1 \mathrm{dr}) . \mathrm{R}_{\mathrm{f}}=0.52$ (n-pentane/EtOAc, 1:2). ${ }^{1} \mathrm{H}$ NMR $(600 \mathrm{MHz}$, $\mathrm{CDCl}_{3}$, mixture of diastereomers) $\delta 7.96-7.87(\mathrm{~m}, 2 \mathrm{H}), 7.71-7.62(\mathrm{~m}, 1 \mathrm{H})$, 7.61-7.50 (m, 2H), 7.42-7.28 (m, 2H), 7.21-7.07 (m, 2H), [6.46 (s), $6.44(\mathrm{~s})$, $1 \mathrm{H}],[6.37(\mathrm{~s}), 6.35(\mathrm{~s}), 1 \mathrm{H}],[6.00(\mathrm{~s}), 5.87(\mathrm{~s}), 1 \mathrm{H}],[3.66(\mathrm{~s}), 3.65(\mathrm{~s}), 3 \mathrm{H}],[3.28(\mathrm{~s}), 3.26(\mathrm{~s}), 3 \mathrm{H}] .{ }^{13} \mathrm{C}\left\{{ }^{1} \mathrm{H}\right\}$ NMR (151 MHz, $\mathrm{CDCl}_{3}$, mixture of diastereomers) $\delta 165.2,165.1,157.1,156.4,140.5,140.2,139.2,139.0$, $138.2,138.1,134.09,134.07,131.2,130.5,130.4,129.9,129.80,129.76,129.7,127.3,127.2,126.6,126.4$, 126.2, 126.0, 122.34, 122.26, 74.14, 74.06, 52.0, 51.9, 44.8, 44.6. IR (ATR): $v=2950,2100,1693,1439$, 1235, 973, 723. MS (ESI): ([M+Na $\left.]^{+}\right)$475.9958. HRMS (ESI): $m / z$ calcd. for $\mathrm{C}_{19} \mathrm{H}_{18} \mathrm{O}_{5} \mathrm{NBrSNa}^{+}: 473.9981$, found: 473.9978 .

$N$-\{[(2-(Methoxycarbonyl)-1-(2-bromophenyl)allyl)oxy]carbonyl $\}-S$-methyl-S-phenylsulfoximine (3ag)<smiles>C=C(OC)C(OC(=O)N=S(C)(=O)c1ccccc1)c1ccccc1Br</smiles>

Prepared from 1a and $\mathbf{2 g}$ following the general procedure; colorless oil (34.3 $\mathrm{mg}, 76 \%$ yield, $1.2: 1 \mathrm{dr}) . \mathrm{R}_{\mathrm{f}}=0.58$ (n-pentane/EtOAc, $\left.1: 2\right) .{ }^{1} \mathrm{H}$ NMR $(600$ $\mathrm{MHz}, \mathrm{CDCl}_{3}$, mixture of diastereomers) $\delta$ 7.97-7.93 (m, 1H), 7.93-7.89 (m, $1 \mathrm{H}), 7.68-7.61(\mathrm{~m}, 1 \mathrm{H}), 7.60-7.51(\mathrm{~m}, 3 \mathrm{H}), 7.45-7.41(\mathrm{~m}, 1 \mathrm{H}), 7.32-7.20(\mathrm{~m}$, 1H), 7.18-7.10 (m, 1H), [6.86 (s), $6.84(\mathrm{~s}), 1 \mathrm{H}],[6.43(\mathrm{~s}), 6.42(\mathrm{~s}), 1 \mathrm{H}],[5.66(\mathrm{~s}), 5.61(\mathrm{~s}), 1 \mathrm{H}],[3.70(\mathrm{~s})$, $3.68(\mathrm{~s}), 3 \mathrm{H}],[3.29$ (s), $3.28(\mathrm{~s}), 3 \mathrm{H}] .{ }^{13} \mathrm{C} \mathrm{NMR}\left(151 \mathrm{MHz}, \mathrm{CDCl}_{3}\right.$, mixture of diastereomers) $\delta 165.40$, $165.35,157.0,156.8,138.4,138.2$, 138.14, 138.13, 137.4, 137.2, 134.0, 133.9, 133.0, 132.9, 129.7, 129.63, $129.56,128.74,128.71,128.3,128.0,127.44,127.37,127.3,123.73,123.70,74.0,52.0,44.63,44.57$. IR $(\mathrm{ATR}): v=3020,2952,2099,1932,1717,1676,1581,1470,1441,1404,1326,1232,1144,1086,972,904$, 843, 733, 685. MS (ESI): ([M+Na] $]^{+}$475.9962. HRMS (ESI): $m / z$ calcd. for $\mathrm{C}_{19} \mathrm{H}_{18} \mathrm{O}_{5} \mathrm{NBrSNa}^{+}: 473.9981$, found: 473.9982 . 
$N$-\{[(2-(Methoxycarbonyl)-1-(4-nitrophenyl)allyl)oxy]carbonyl $\}-S$-methyl-S-phenylsulfoximine (3ah)<smiles>C=C(C(C)=O)C(OC(=O)N=S(C)(=O)c1ccccc1)c1ccc([N+](=O)[O-])cc1</smiles>

Prepared from 1a and $\mathbf{2 h}$ following the general procedure; yellow oil $(21.3 \mathrm{mg}$, $51 \%$ yield, 1.3:1 dr). $\mathrm{R}_{\mathrm{f}}=0.54$ ( $n$-pentane/EtOAc, 1:2). ${ }^{1} \mathrm{H}$ NMR $(600 \mathrm{MHz}$, $\mathrm{CDCl}_{3}$, mixture of diastereomers) $\delta[8.17-8.14(\mathrm{~m}), 8.09-8.06(\mathrm{~m}), 1 \mathrm{H}], 8.10$ $8.04(\mathrm{~m}, 1 \mathrm{H}), 7.97-7.91(\mathrm{~m}, 2 \mathrm{H}), 7.73-7.64(\mathrm{~m}, 1 \mathrm{H}), 7.61-7.55(\mathrm{~m}, 3 \mathrm{H}), 7.42-$ $7.36(\mathrm{~m}, 1 \mathrm{H}),[6.57(\mathrm{~s}), 6.56(\mathrm{~s}), 1 \mathrm{H}],[6.42(\mathrm{~s}), 6.38(\mathrm{~s}), 1 \mathrm{H}],[6.08(\mathrm{~s}), 5.92(\mathrm{~s})$ $1 \mathrm{H}], 3.67(\mathrm{~s}, 3 \mathrm{H}),[3.30(\mathrm{~s}), 3.28(\mathrm{~s}), 3 \mathrm{H}] .{ }^{13} \mathrm{C}\left\{{ }^{1} \mathrm{H}\right\}$ NMR (151 MHz, $\mathrm{CDCl}_{3}$, mixture of diastereomers) $\delta$ $165.0,156.9,156.4,147.5,145.6,145.3,138.7,138.6,138.2,138.0,134.2,134.1,129.80,129.78,128.45$, $128.39,127.3,126.8,126.6,123.6,123.5,73.8,52.0,44.8,44.7$. IR (ATR): $v=3019,2946,2255,2103$, 1924, 1713, 1606, 1520, 1444, 1401, 1345, 1236, 1144, 1087, 974, 907, 855, 731. MS (ESI): ([M+Na $\left.]^{+}\right)$ 441.0731. HRMS (ESI): $m / z$ calcd. for $\mathrm{C}_{19} \mathrm{H}_{18} \mathrm{O}_{7} \mathrm{~N}_{2} \mathrm{SNa}^{+}$: 441.0727, found: 441.0720 .

\section{$N$-\{[(2-(Methoxycarbonyl)-1-[4-(trifluoromethyl)phenyl]allyl)oxy]carbonyl $\}-S$ - methyl-S-phenylsulfoximine (3ai)}

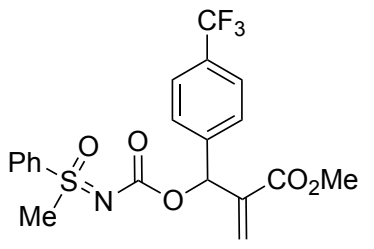

Prepared from 1a and $\mathbf{2} \mathbf{i}$ following the general procedure; white solid (26.9 $\mathrm{mg}, 61 \%$ yield, 1.2:1 dr). $\mathrm{R}_{\mathrm{f}}=0.57$ (n-pentane/EtOAc, 1:2). ${ }^{1} \mathrm{H}$ NMR (600 $\mathrm{MHz}, \mathrm{CDCl}_{3}$, mixture of diastereomers) $\delta 77.94-7.88(\mathrm{~m}, 2 \mathrm{H}), 7.70-7.63(\mathrm{~m}$, 1H), 7.59-7.44 (m, 5H), $7.32(\mathrm{~d}, J=8.1 \mathrm{~Hz}, 1 \mathrm{H}),[6.55(\mathrm{~s}), 6.53(\mathrm{~s}), 1 \mathrm{H}],[6.39$ (s), 6.35 (s), 1H], [6.04 (s), 5.89 (s), 1H], [3.664 (s), 3.658 (s), 3H], [3.29 (s), $3.26(\mathrm{~s}), 3 \mathrm{H}] .{ }^{13} \mathrm{C}\left\{{ }^{1} \mathrm{H}\right\}$ NMR (151 MHz, $\mathrm{CDCl}_{3}$, mixture of diastereomers) $\delta 165.1,157.0,156.5,142.3$, 142.0, 139.1, 138.9, 138.3, 138.1, 134.1, 134.0, 130.2 (q, $\left.J_{C-F}=32.2 \mathrm{~Hz}\right), 130.1$ (q, $\left.J_{C-F}=32.7 \mathrm{~Hz}\right), 129.7$, 127.93, 127.90, 127.3, 127.2, 126.3, 126.2, $125.3\left(\mathrm{q}, J_{C-F}=3.7 \mathrm{~Hz}\right), 125.2\left(\mathrm{q}, J_{C-F}=3.7 \mathrm{~Hz}\right), 124.01\left(\mathrm{q}, J_{C-F}\right.$ $=272.3 \mathrm{~Hz}$ ), 123.98 (q, $J_{C-F}=272.2 \mathrm{~Hz}$ ), 74.2, 74.1, 51.96, 51.97, 44.8, 44.6. IR (ATR): $v=3020,2954$, 2103, 1924, 1716, 1676, 1629, 1583, 1444, 1416, 1323, 1236, 1159, 1115, 1066, 975, 907, 854, 782, 742, 686. MS (ESI): $\left([\mathrm{M}+\mathrm{Na}]^{+}\right)$464.0746. HRMS (ESI): $\mathrm{m} / z$ calcd. for $\mathrm{C}_{20} \mathrm{H}_{18} \mathrm{O}_{5} \mathrm{NF}_{3} \mathrm{SNa}^{+}$: 464.0750, found: 464.0748 .

$N$-\{[(2-(Methoxycarbonyl)-1-(naphthalen-1-yl)allyl)oxy]carbonyl $\}-S$-methyl-S-phenylsulfoximine (3aj)<smiles>C=C(OC)C(OC(=O)N=S(C)(=O)c1ccccc1)c1cccc2ccccc12</smiles>

Prepared from $\mathbf{1 a}$ and $\mathbf{2} \mathbf{j}$ following the general procedure; white solid (29.6 $\mathrm{mg}$, $70 \%$ yield, 1.3:1 dr). $\mathrm{R}_{\mathrm{f}}=0.44$ ( $n$-pentane/EtOAc, 1:2). ${ }^{1} \mathrm{H}$ NMR $(600 \mathrm{MHz}$, $\mathrm{CDCl}_{3}$, mixture of diastereomers) $\delta 8.06-7.98(\mathrm{~m}, 1 \mathrm{H}), 7.93-7.73(\mathrm{~m}, 4 \mathrm{H})$,

7.67-7.32 (m, 8H), [6.47 (s), $6.44(\mathrm{~s}), 1 \mathrm{H}],[5.88(\mathrm{~s}), 5.77(\mathrm{~s}), 1 \mathrm{H}],[3.66(\mathrm{~s})$, $3.64(\mathrm{~s}), 1 \mathrm{H}],[3.24(\mathrm{~d}, J=0.6 \mathrm{~Hz}), 3.22(\mathrm{~d}, J=0.6 \mathrm{~Hz}), 3 \mathrm{H}] .{ }^{13} \mathrm{C}\left\{{ }^{1} \mathrm{H}\right\} \mathrm{NMR}\left(151 \mathrm{MHz}, \mathrm{CDCl}_{3}\right.$, mixture of diastereomers) $\delta 165.72,165.69,157.4,156.9,139.21,139.15,138.2,138.1,133.9,133.81,133.76,133.6$, $133.5,131.0,129.6$, 129.3, 129.0, 128.9, 128.6, 128.5, 127.8, 127.4, 127.0, 126.4, 125.7, 125.6, 125.4, 125.3, $125.2,125.1,123.7,71.5,71.4,52.00,51.96,44.64,44.59$. IR (ATR): $v=3032,2261,2094,1928,1689$, 1436, 1236, 967, 740. HRMS (ESI): $m / z$ calcd. for $\mathrm{C}_{23} \mathrm{H}_{21} \mathrm{O}_{5} \mathrm{NSNa}^{+}: 446.1033$, found: 446.1023 .

\section{$N$-\{[(2-(Ethoxycarbonyl)-1-phenylallyl)oxy]carbonyl\}-S-methyl-S-phenylsulfoximine (3ak)}<smiles>C=C(OCC)C(OC(=O)N=S(C)(=O)c1ccccc1)c1ccccc1</smiles>

Prepared from 1a and $\mathbf{2 k}$ following the general procedure; white solid (34.4 mg, $89 \%$ yield, 1.2:1 dr). $\mathrm{R}_{\mathrm{f}}=0.42$ (n-pentane/EtOAc, $\left.1: 2\right) .{ }^{1} \mathrm{H}$ NMR $(400 \mathrm{MHz}$, $\mathrm{CDCl}_{3}$, mixture of diastereomers) $\delta 7.94-7.87(\mathrm{~m}, 2 \mathrm{H}), 7.67-7.60(\mathrm{~m}, 1 \mathrm{H})$, 7.58-7.50 (m, 2H), 7.38-7.34 (m, 1H), 7.31-7.18 (m, 4H), [6.52 (s), $6.50(\mathrm{~s}), 1 \mathrm{H}],[6.34-6.33(\mathrm{~m}), 6.32-$ $6.30(\mathrm{~m}), 1 \mathrm{H}],[5.95-5.93(\mathrm{~m}), 5.84-5.82(\mathrm{~m}), 1 \mathrm{H}], 4.16-4.03(\mathrm{~m}, 2 \mathrm{H}),[3.26(\mathrm{~s}), 3.25(\mathrm{~s}), 3 \mathrm{H}], 1.21-1.06$ 
(m, 3H). ${ }^{13} \mathrm{C}\left\{{ }^{1} \mathrm{H}\right\}$ NMR (151 MHz, $\mathrm{CDCl}_{3}$, mixture of diastereomers) $\delta 164.97,164.96,157.3,156.9,140.0$, $139.9,138.3,138.2$, 138.1 137.9, 134.0, 133.9, 129.7, 128.25, 128.17, 128.1, 128.0, 127.81, 127.78, 127.4, $127.3,125.3,125.2,75.05,75.00,60.8,44.8,44.6,13.99,13.97$. IR (ATR): $v=2924,2859,2320,2095$, $1918,1699,1480,1450,1406,1374,1333,1232,1154,1102,981,904,797,752,696 . \mathrm{MS}(\mathrm{ESI}):\left([\mathrm{M}+\mathrm{Na}]^{+}\right)$ 410.1037. HRMS (ESI): $m / z$ calcd. for $\mathrm{C}_{20} \mathrm{H}_{21} \mathrm{O}_{5} \mathrm{NSNa}^{+}: 410.1033$, found: 410.1032 .

\section{$N$-\{[(2-(Benzyloxycarbonyl)-1-phenylallyl)oxy]carbonyl $\}-S$-methyl-S-phenylsulfoximine (3al)}<smiles>C=C(C(=O)OCc1ccccc1)C(OC(=O)N=S(C)(=O)c1ccccc1)c1ccccc1</smiles>

Prepared from 1a and $\mathbf{2 l}$ following the general procedure; colorless oil $(20.2$ $\mathrm{mg}, 45 \%$ yield, 1.3:1 dr). $\mathrm{R}_{\mathrm{f}}=0.69$ (n-pentane/EtOAc, $\left.1: 2\right) .{ }^{1} \mathrm{H}$ NMR $(600$

$\mathrm{MHz}, \mathrm{CDCl}_{3}$, mixture of diastereomers) $\delta[7.92(\mathrm{~d}, J=7.6 \mathrm{~Hz}), 7.90(\mathrm{~d}, J=7.6$ $\mathrm{Hz}), 2 \mathrm{H}], 7.71-7.61(\mathrm{~m}, 1 \mathrm{H}), 7.58-7.50(\mathrm{~m}, 2 \mathrm{H}), 7.39-7.26(\mathrm{~m}, 5 \mathrm{H}), 7.24-7.15(\mathrm{~m}, 5 \mathrm{H})$, [6.56 (s), 6.53 (s), $1 \mathrm{H}],[6.41(\mathrm{~s}), 6.39(\mathrm{~s}), 1 \mathrm{H}],[6.01(\mathrm{~s}), 5.89(\mathrm{~s}), 1 \mathrm{H}), 5.17-5.01(\mathrm{~m}, 2 \mathrm{H}),[3.26(\mathrm{~s}), 3.25(\mathrm{~s}), 3 \mathrm{H}] .{ }^{13} \mathrm{C}\left\{{ }^{1} \mathrm{H}\right\}$ NMR (151 MHz, $\mathrm{CDCl}_{3}$, mixture of diastereomers) $\delta 164.8,157.2,156.8,139.7,139.6,138.3,138.2,138.0$, $137.8,135.5,134.0,133.9,129.7,128.43,128.42,128.3,128.22,128.15,128.08,128.04,128.01,127.85$, 127.82, 127.4, 127.3, 126.0, 125.8, 75.04, 74.98, 66.6, 44.7, 44.6. IR (ATR): $v=3035,2259,1692,1446$, 1238, 1130, 967, 721. MS (ESI): $\left([\mathrm{M}+\mathrm{Na}]^{+}\right)$472.1168. HRMS (ESI): $m / z$ calcd. for $\mathrm{C}_{25} \mathrm{H}_{23} \mathrm{O}_{5} \mathrm{NSNa}^{+}$: 472.1189, found: 472.1175 .

\section{$N$-\{[(2-(Methoxycarbonyl)-1-(cyclohexyl)allyl)oxy]carbonyl $\}-S, S$-diphenylsulfoximine (3Im)}

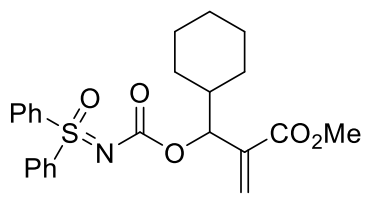

Prepared from $\mathbf{1 l}$ and $\mathbf{2} \mathbf{m}$ on a $0.3 \mathrm{mmol}$ scale following the general procedure; yellow oil (27.1 mg, $21 \%$ yield). $\mathrm{R}_{\mathrm{f}}=0.38$ (dichloromethane/ $\left.\mathrm{Et}_{2} \mathrm{O}, 20: 1\right) .{ }^{1} \mathrm{H}$ NMR (600 MHz, $\left.\mathrm{CDCl}_{3}\right)$ ઈ 8.06-7.98 (m, 4H), 7.61-7.55 (m, 2H), 7.55-7.48 $(\mathrm{m}, 4 \mathrm{H}), 6.24(\mathrm{~d}, J=1.2 \mathrm{~Hz}, 1 \mathrm{H}), 5.65$ (t, $J=1.2 \mathrm{~Hz}, 1 \mathrm{H}), 5.38$ (dd, $J=4.9$, $1.1 \mathrm{~Hz}, 1 \mathrm{H}), 3.71(\mathrm{~s}, 3 \mathrm{H}), 1.65-1.43\left(\mathrm{~m}, 5 \mathrm{H}\right.$ plus overlapping $\mathrm{H}_{2} \mathrm{O}$ signal), $1.16-0.66(\mathrm{~m}, 6 \mathrm{H}) .{ }^{13} \mathrm{C}\left\{{ }^{1} \mathrm{H}\right\}$ NMR (151 MHz, $\left.\mathrm{CDCl}_{3}\right) \delta 166.2,157.0,140.3,140.0,139.2,133.52,133.49,129.7,129.6,127.9,127.8$, $126.1,77.7,52.0,41.2,29.2,27.0,26.24,26.19,26.1$. IR (ATR): $v=2928,2854,21611711,1446,1235$, 1154, 1092, 1071, 974, 891, 818, 733, 688. MS (EI): $441\left(2, \mathrm{M}^{+}\right), 244$ (100). HRMS (ESI): $m / z$ calcd. for $\mathrm{C}_{24} \mathrm{H}_{27} \mathrm{O}_{5} \mathrm{NSNa}^{+}: 464.1502$, found: 464.1510 .

\section{(E)-Imino(methyl)(styryl)- $\lambda^{6}$-sulfanone (1i)}

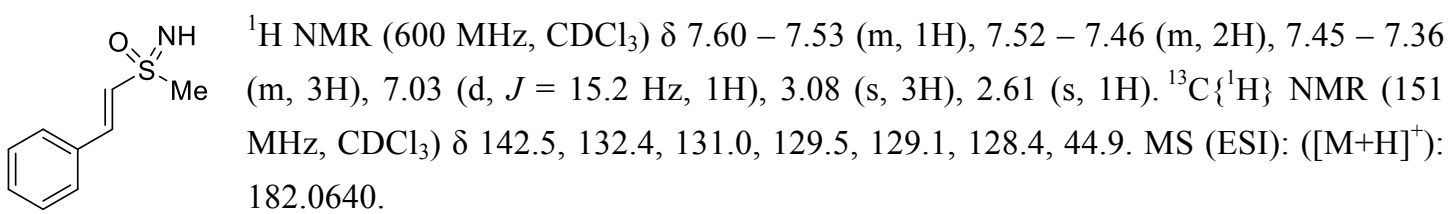

\section{References}

(1) (a) Okamura, H.; Bolm, C. Org. Lett. 2004, 6, 1305. (b) Mancheño, O. G.; Bistri, O.; Bolm, C. Org. Lett. 2007, 9, 3809. (c) Eis, K.; Prien, O.; Luecking, U.; Guenther, J.; Zopf, D.; Brohm, D.; Vöhringer, V.; Woltering, E.; Beck, H.; Lobell, M.; Li, V. M.-J.; Greschat, S. Patent Appl. WO 2008141843 A1, 2008. (d) Yadav, M. R.; Rit, R. K.; Sahoo, A. K. Chem. Eur. J. 2012, 18, 5541. (e) Zenzola, M.; Doran, R.; Degennaro, L.; Luisi, R.; Bull, J. A. Angew. Chem., Int. Ed. 2016, 55, 7203. (f) Tota, A.; Zenzola, M.; Chawner, S. J.; St. John-Campbell, S.; Carlucci, C.; Romanazzi, G.; Degennaro, L.; Bull, J. A.; Luisi, R. Chem. Commun. 2017, 53, 348.

(2) Feng, J.; Lu, X.; Kong, A.; Han, X. Tetrahedron 2007, 63, 6035. 


\section{5. ${ }^{1} \mathrm{H}$ and ${ }^{13} \mathrm{C}$ NMR Spectra}

$N$-\{[(2-(Methoxycarbonyl)-1-phenylallyl)oxy]carbonyl $\}-S$-methyl-S-phenylsulfoximine (3aa)

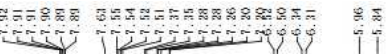

$\mathrm{Me}^{\mathrm{S}^{\prime \prime}} \mathrm{N}_{\mathrm{N}}^{\mathrm{O}} \mathrm{H}_{\mathrm{O}}^{\mathrm{O}} \mathrm{CO}_{2} \mathrm{Me}$

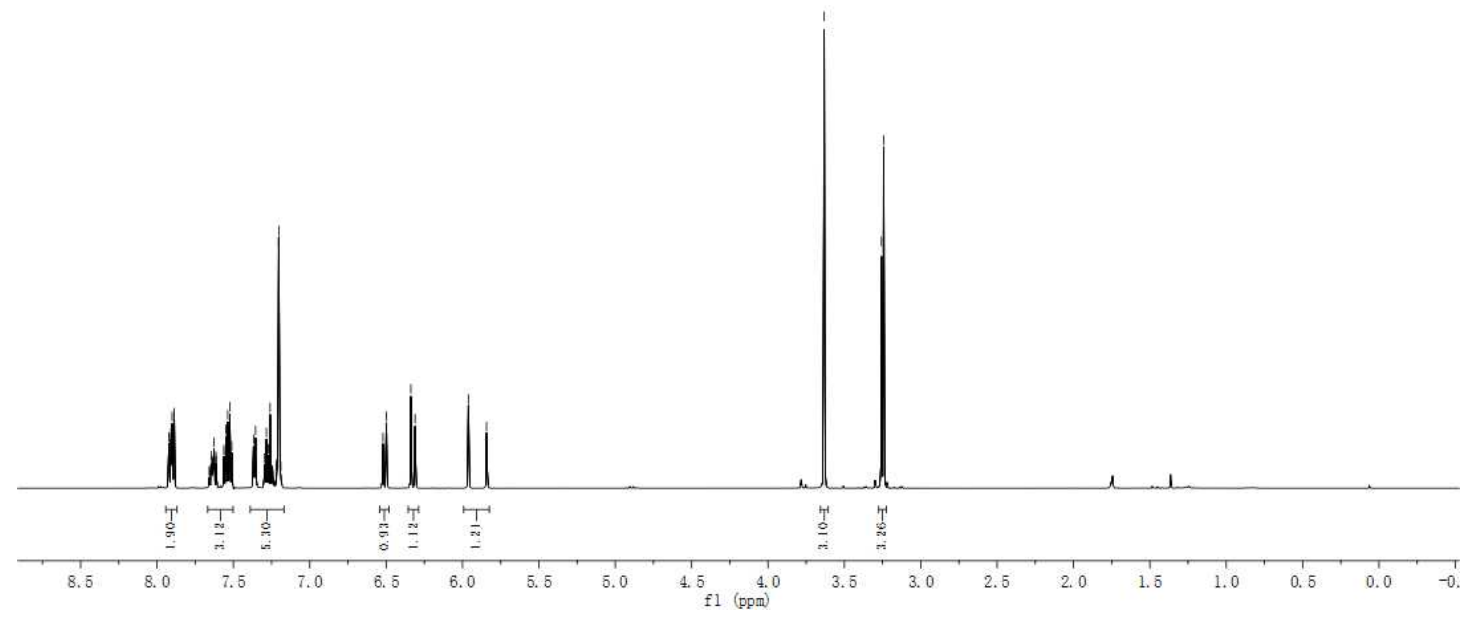

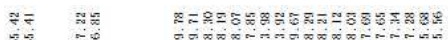

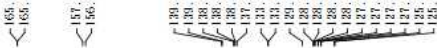

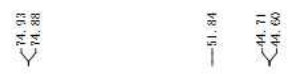

$\mathrm{Me}^{\prime} \mathrm{S}_{\mathrm{N}}^{\mathrm{O}} \mathrm{N}_{\mathrm{O}}^{\mathrm{O}} \mathrm{CO}_{2} \mathrm{Me}$

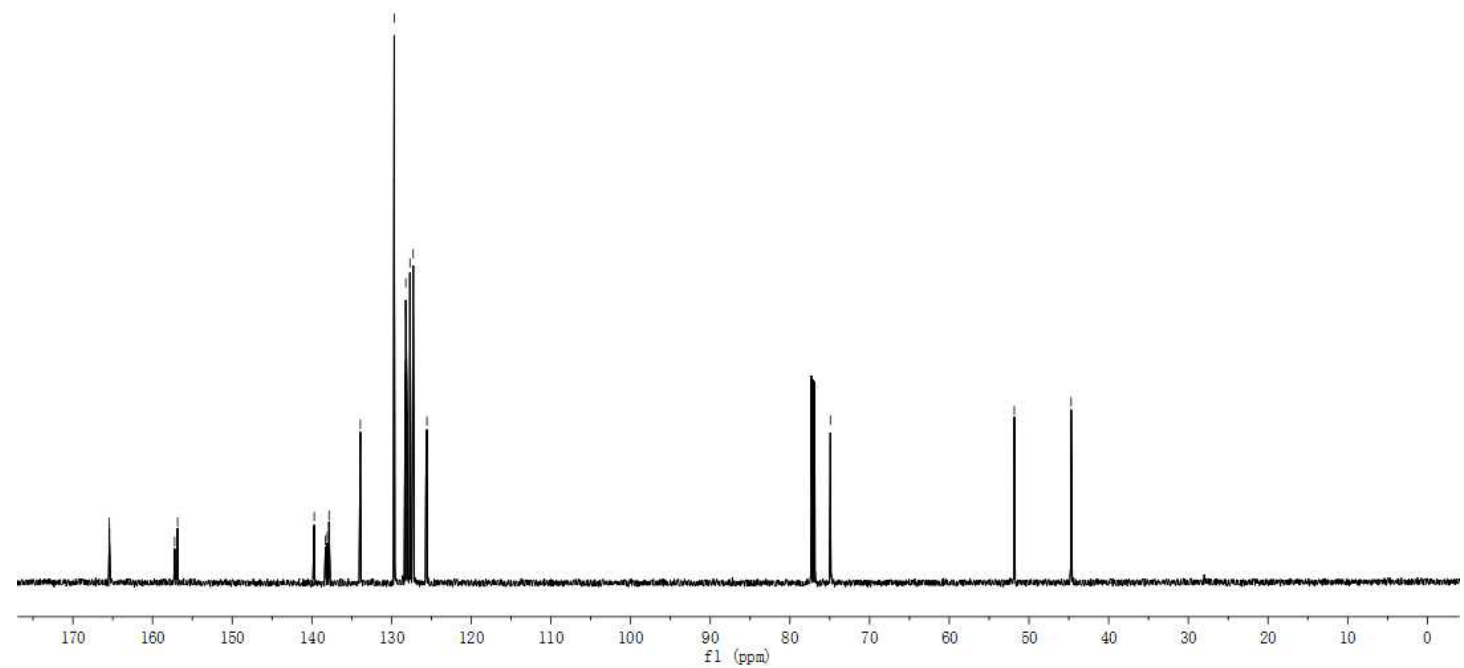


$N$-\{[(2-(Methoxycarbonyl)-1-phenylallyl)oxy]carbonyl $\}-S$-methyl-S-(p-tolyl)sulfoximine (3ba)

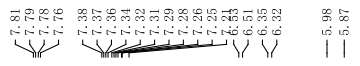

Y)<smiles>C=C(C(C)=O)C(=O)OC(=O)N=S(C)(=O)c1ccc(C)cc1</smiles>

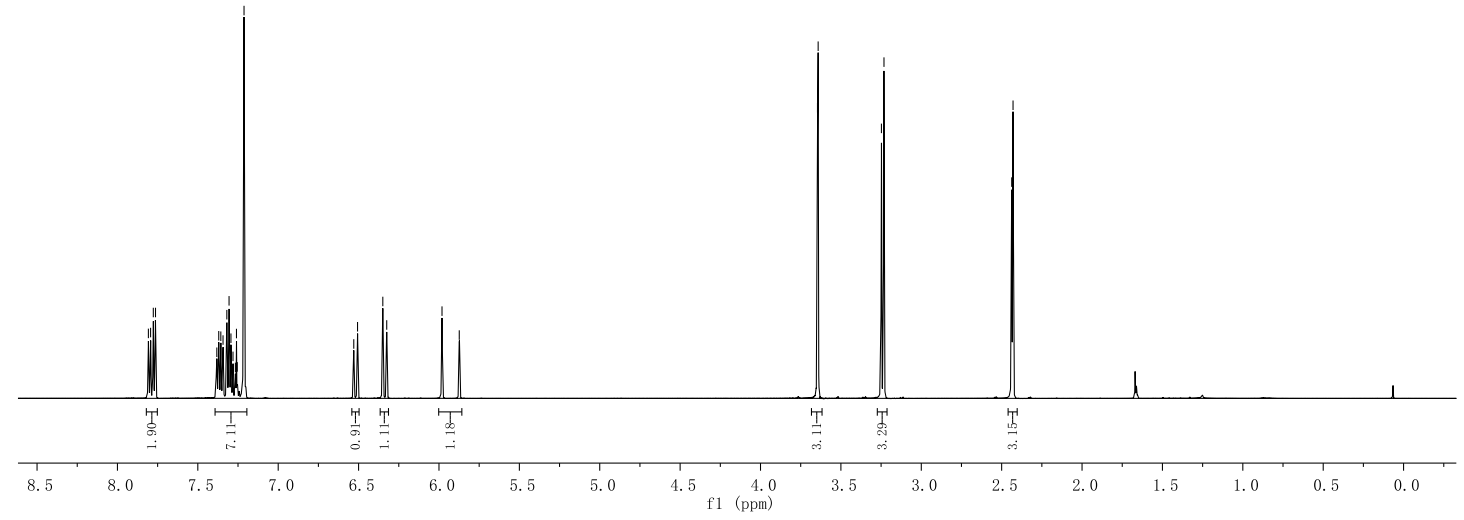

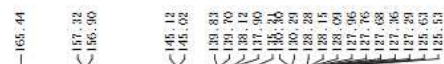

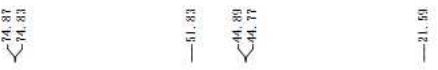

$\mathrm{Me}$<smiles>C=C(C(C)=O)C(OC(=O)N=S(C)(=O)c1ccc(C)cc1)c1ccccc1</smiles>

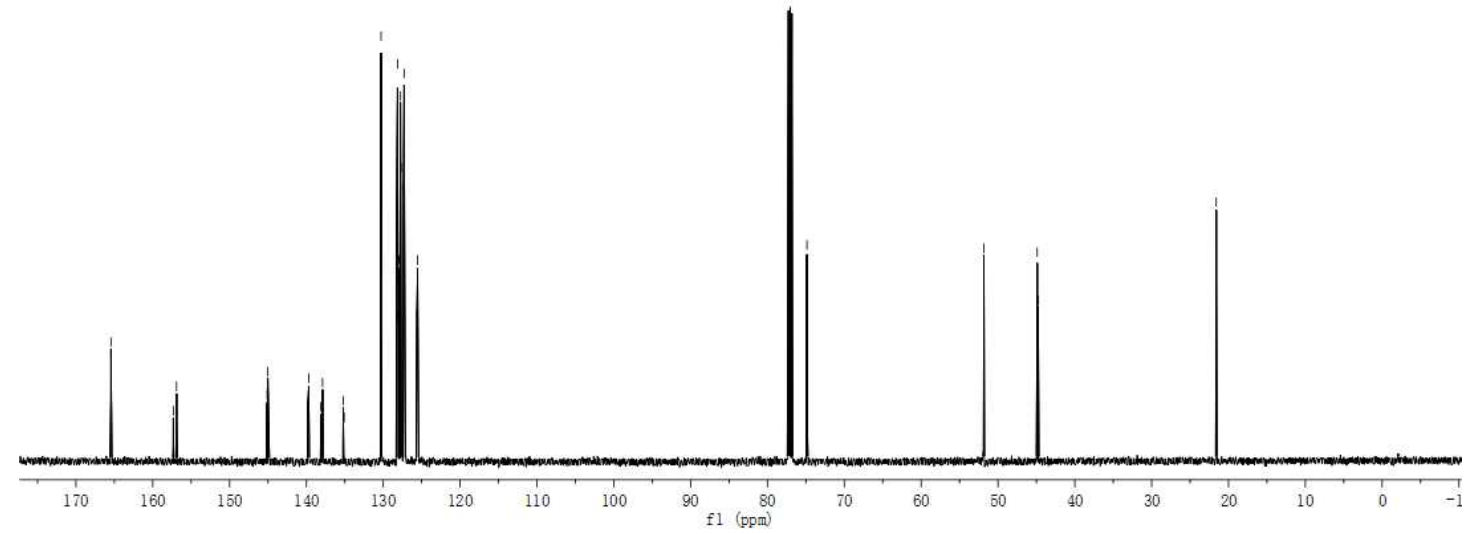


$N$-\{[(2-(Methoxycarbonyl)-1-phenylallyl)oxy]carbonyl $\}-S$-methyl-S-(4-fluorophenyl)sulfoximine (3ca)

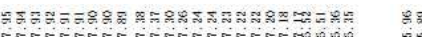

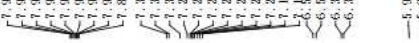

80
$i$<smiles>C=C(N=S(C)(=O)c1ccc(F)cc1)C(=O)OC(C(=O)OC)c1ccccc1</smiles>

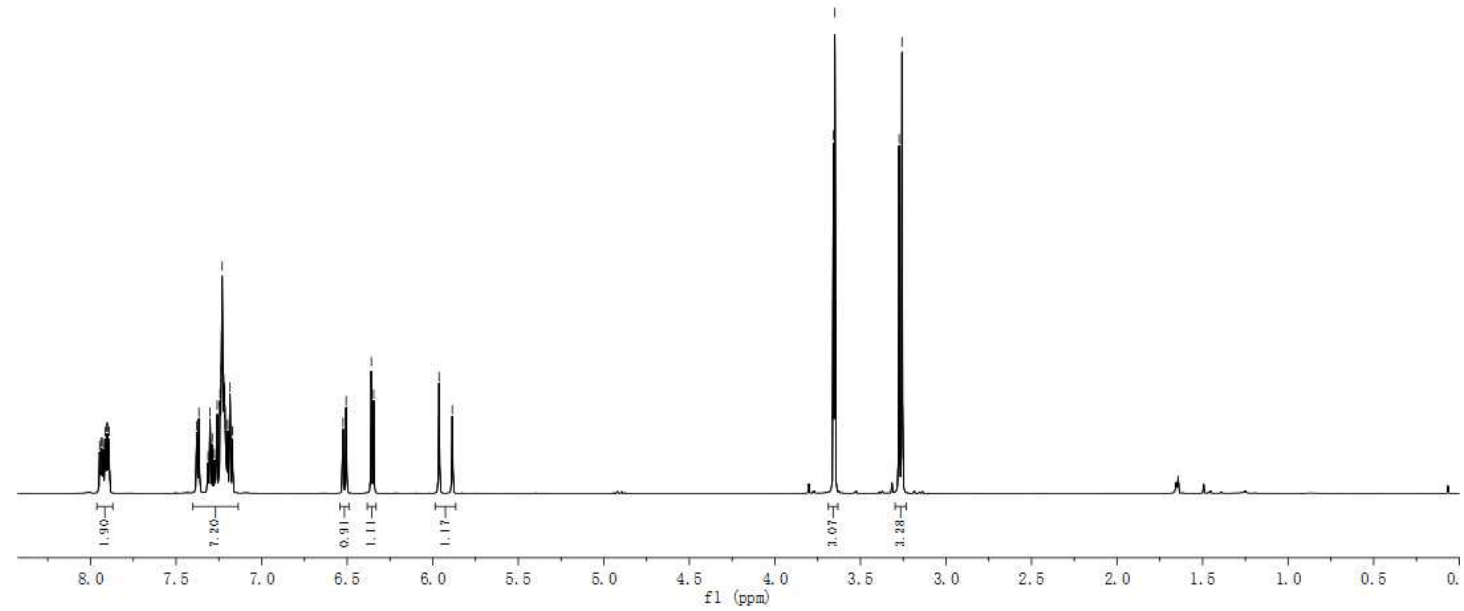

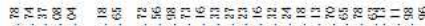

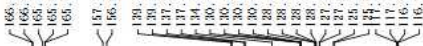

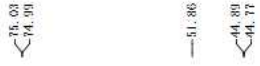

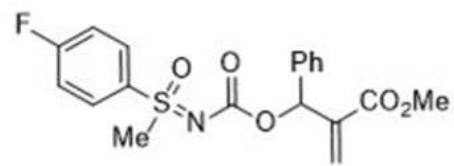

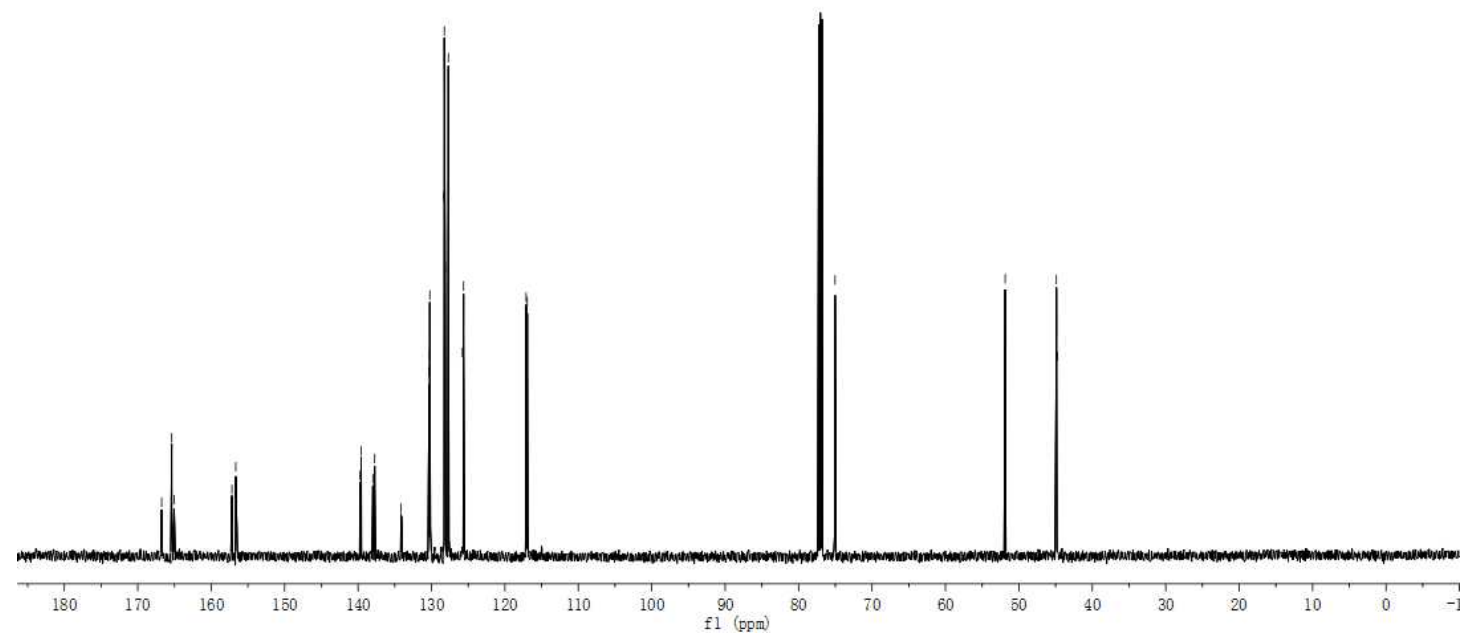


$N$-\{[(2-(Methoxycarbonyl)-1-phenylallyl)oxy]carbonyl $\}$-S-methyl-S-(4-chlorophenyl)sulfoximine (3da)

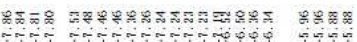

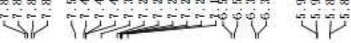

8
80

$\underbrace{\mathrm{Cl}}_{\mathrm{Me}^{\prime}{ }_{\mathrm{N}}^{\prime \prime}} \mathrm{ll}_{\mathrm{O}}^{\mathrm{Ph}} \mathrm{CO}_{2} \mathrm{Me}$

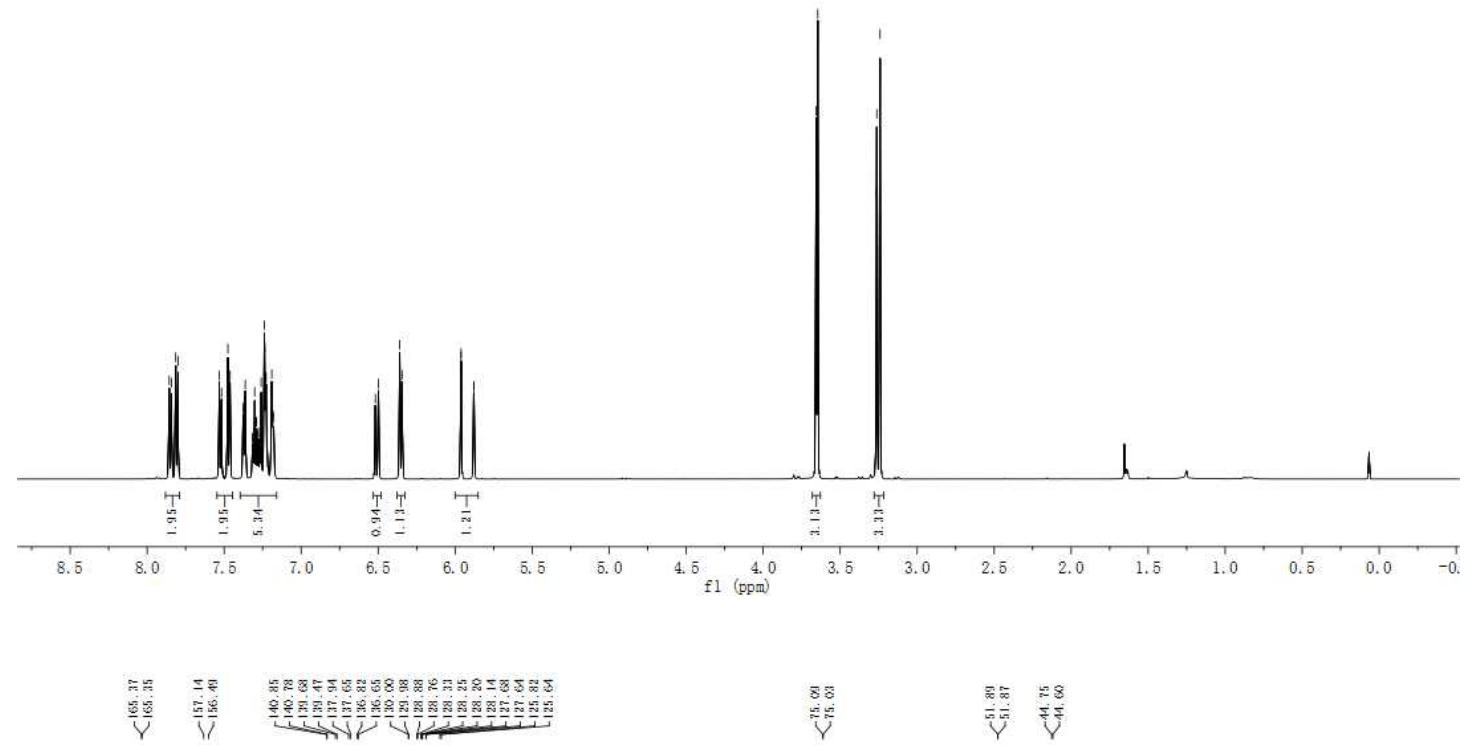

$\overbrace{\mathrm{Me}^{\prime}=\mathrm{N}}^{\mathrm{Cl}} \overbrace{\mathrm{O}}^{\mathrm{C}} \mathrm{CO}_{2}^{\mathrm{Me}}$

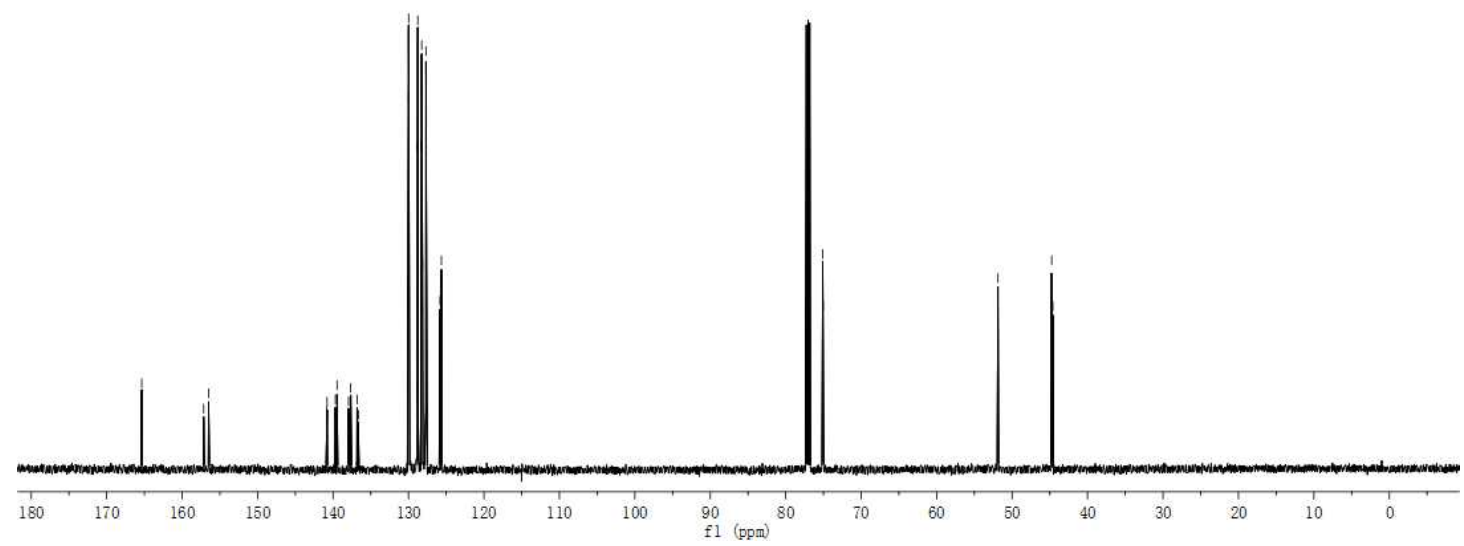


$N$-\{[(2-(Methoxycarbonyl)-1-phenylallyl)oxy]carbonyl $\}-S$-methyl-S-(4-acetylphenyl)sulfoximine (3ea)

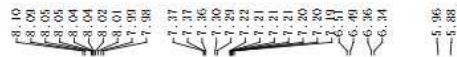

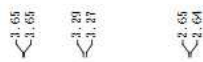<smiles>C=C(OC)C(OC(=O)N=S(C)(=O)c1ccc(C(C)=O)cc1)C(C)=O</smiles>

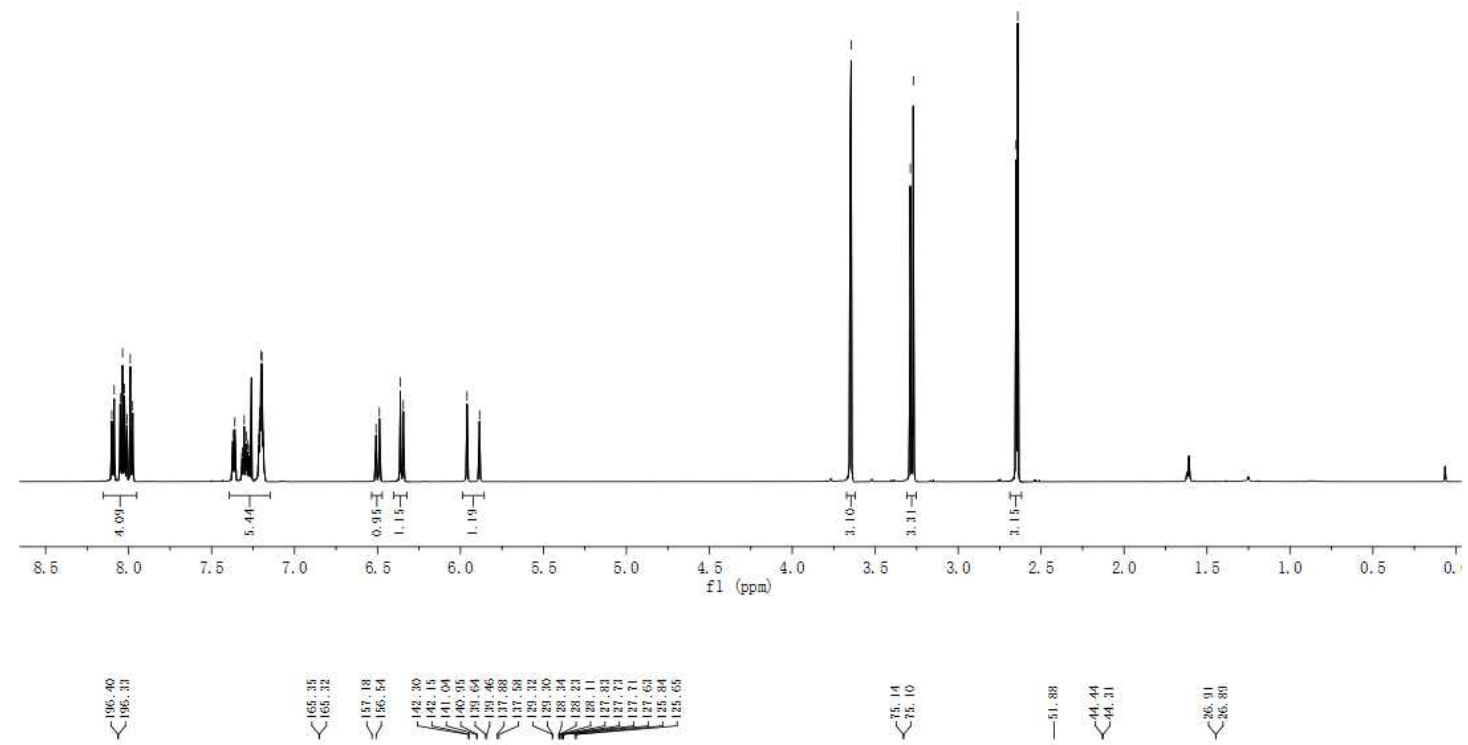<smiles>C=C(N=S(C)(=O)c1ccc(C(C)=O)cc1)C(=O)OC(C(=O)OC)c1ccccc1</smiles>

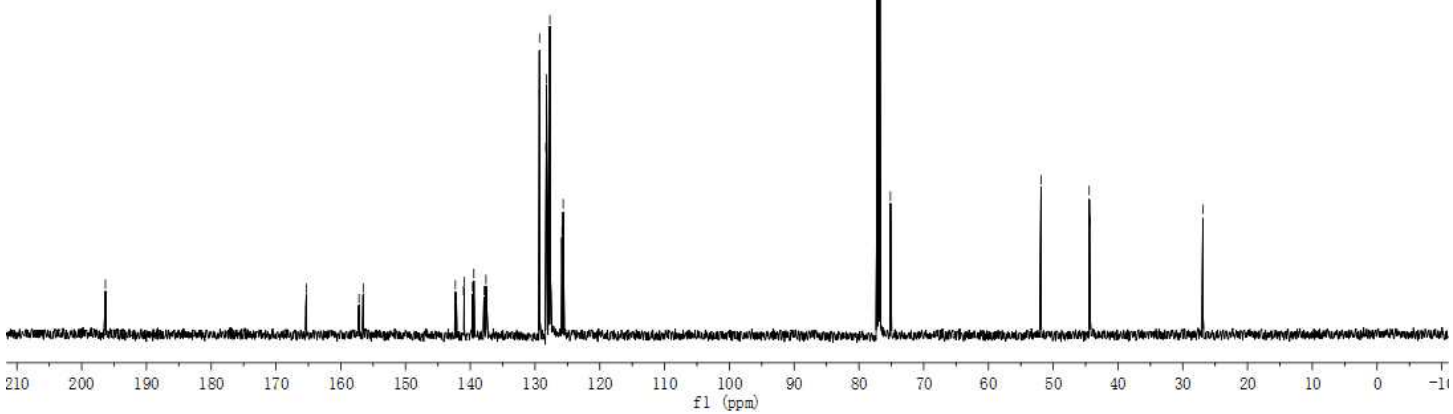


$N$-\{[(2-(Methoxycarbonyl)-1-phenylallyl)oxy]carbonyl $\}-S$-methyl-S-(3-bromophenyl)sulfoximine (3fa)

3 8 万人

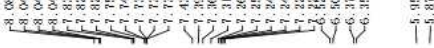

88
$Y$<smiles>C=C(C(=O)OC(=O)N=S(C)(=O)c1cccc(Br)c1)c1ccccc1</smiles>

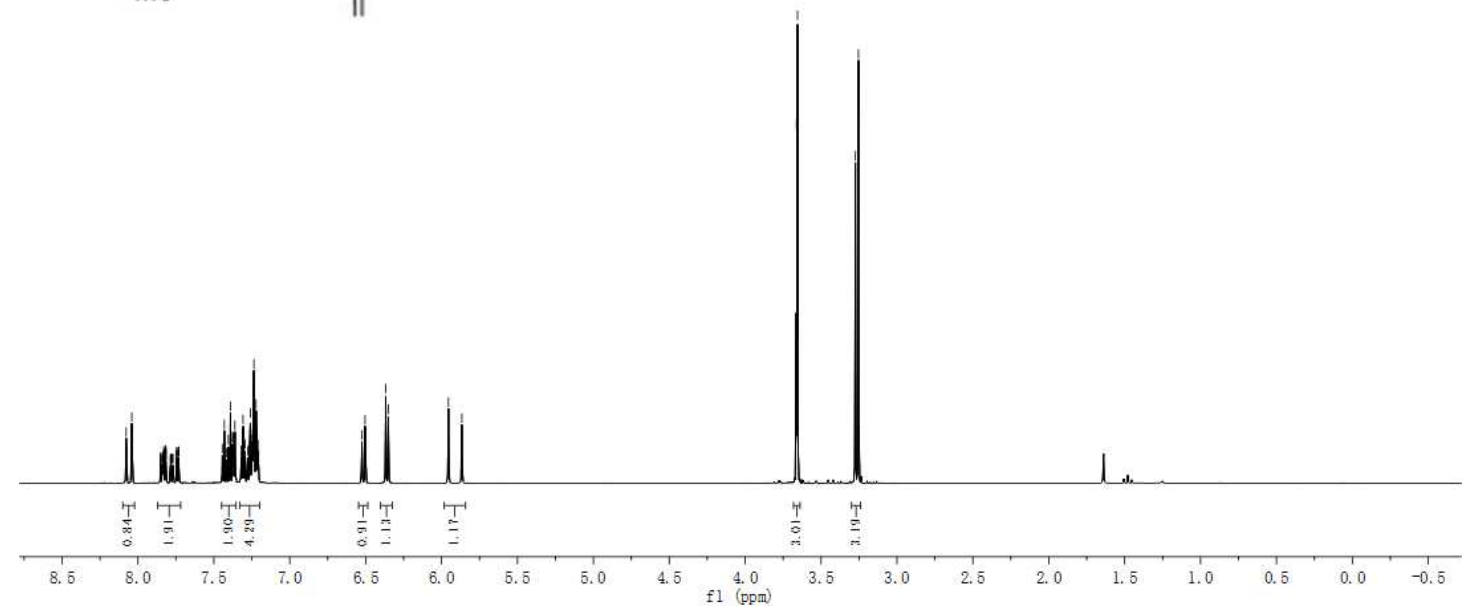

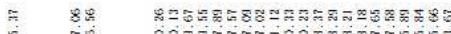

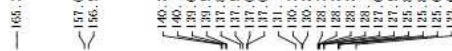

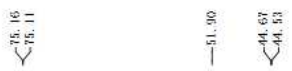

$\mathrm{Me}_{\mathrm{S}_{i=\mathrm{N}}^{\prime \prime}}^{\mathrm{Br}}$

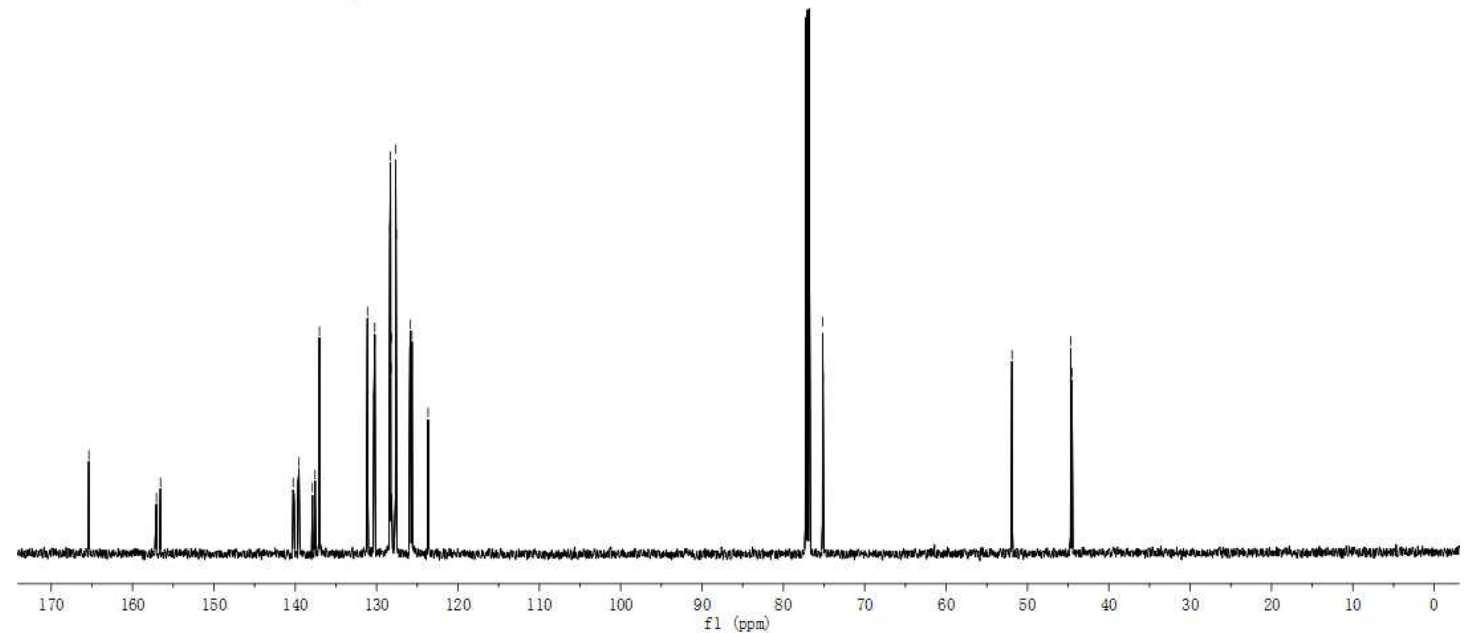


$N$-\{[(2-(Methoxycarbonyl)-1-phenylallyl)oxy]carbonyl $\}-S$-methyl-S-(2-methoxyphenyl)sulfoximine (3ga)

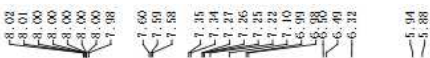<smiles>C=C(C(C)=O)C(=O)OC(N=S(C)(=O)c1ccccc1OC)c1ccccc1</smiles>

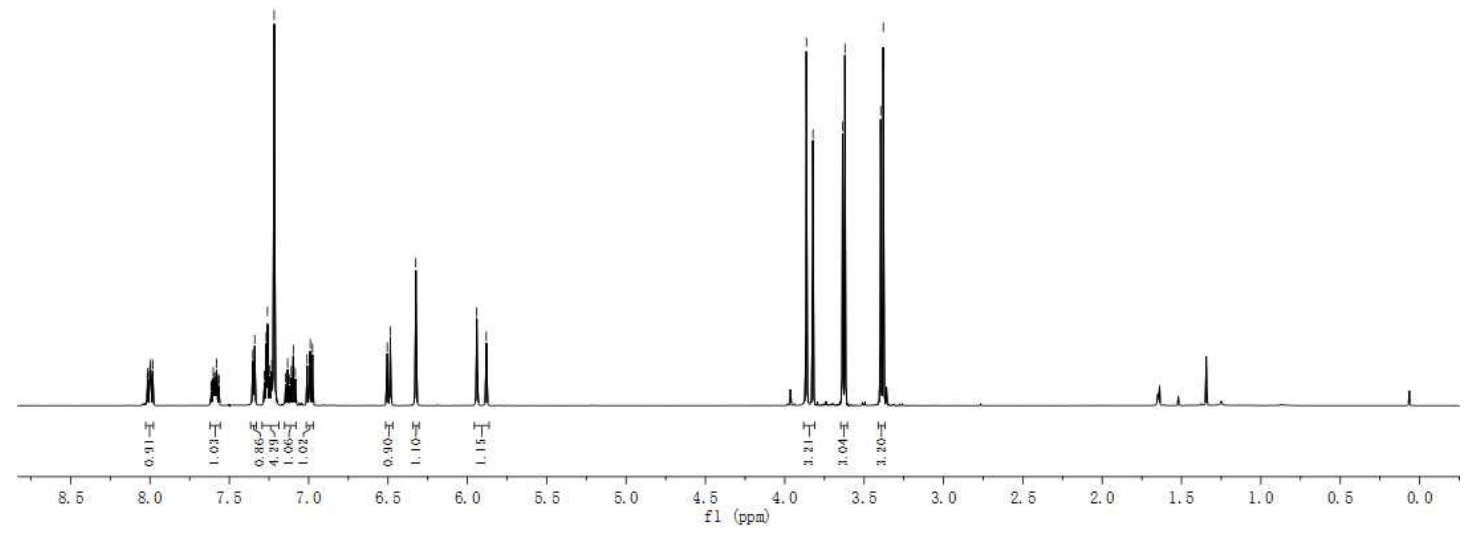

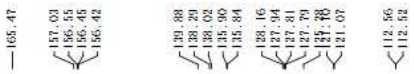
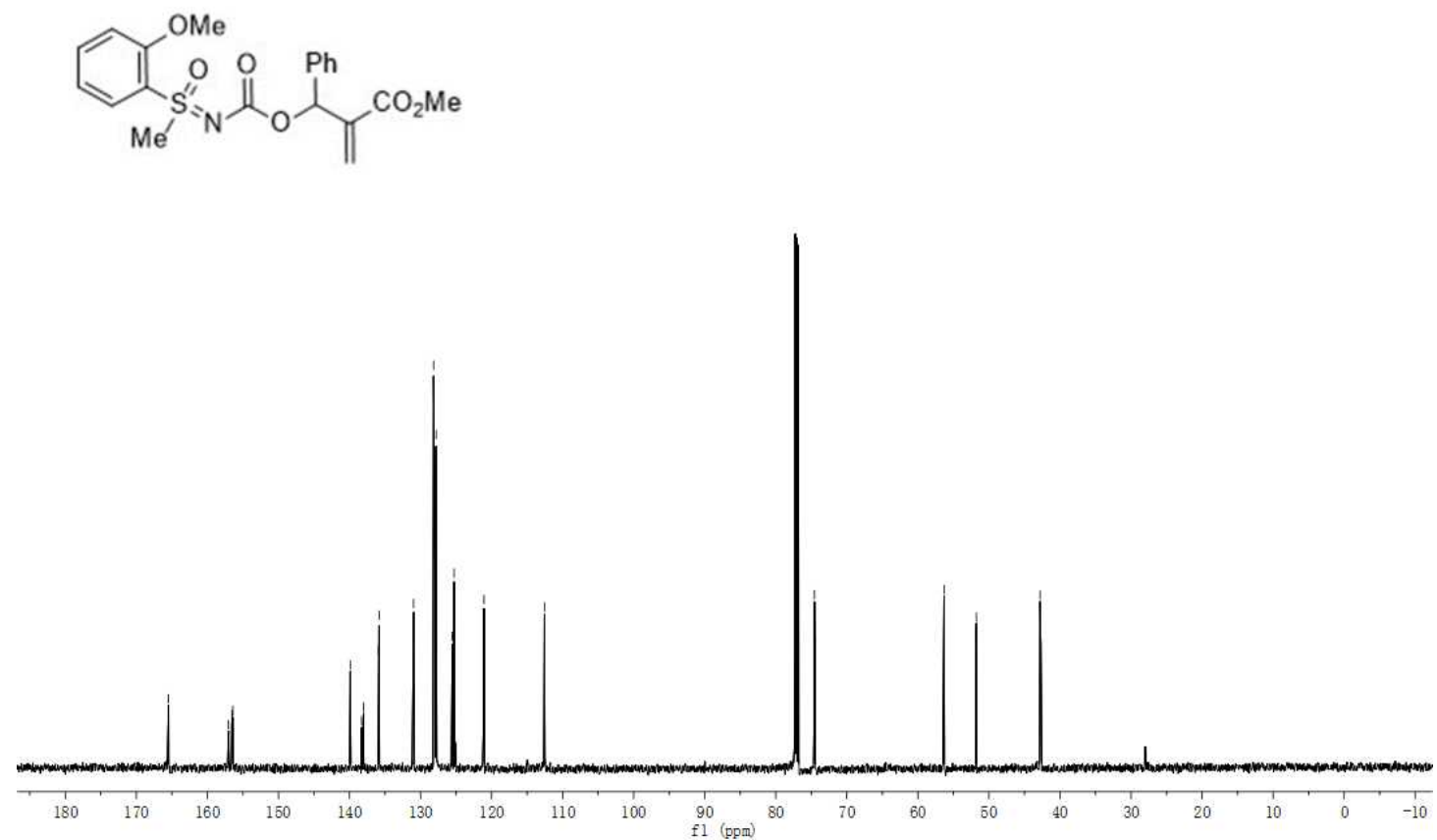
$N$-\{[(2-(Methoxycarbonyl)-1-phenylallyl)oxy]carbonyl $\}-S$-methyl-S-(pyridin-2-yl)sulfoximine (3ha)

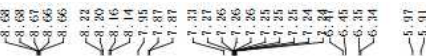

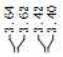

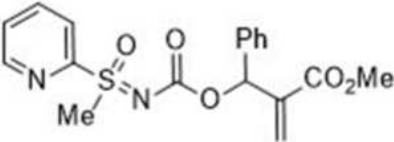

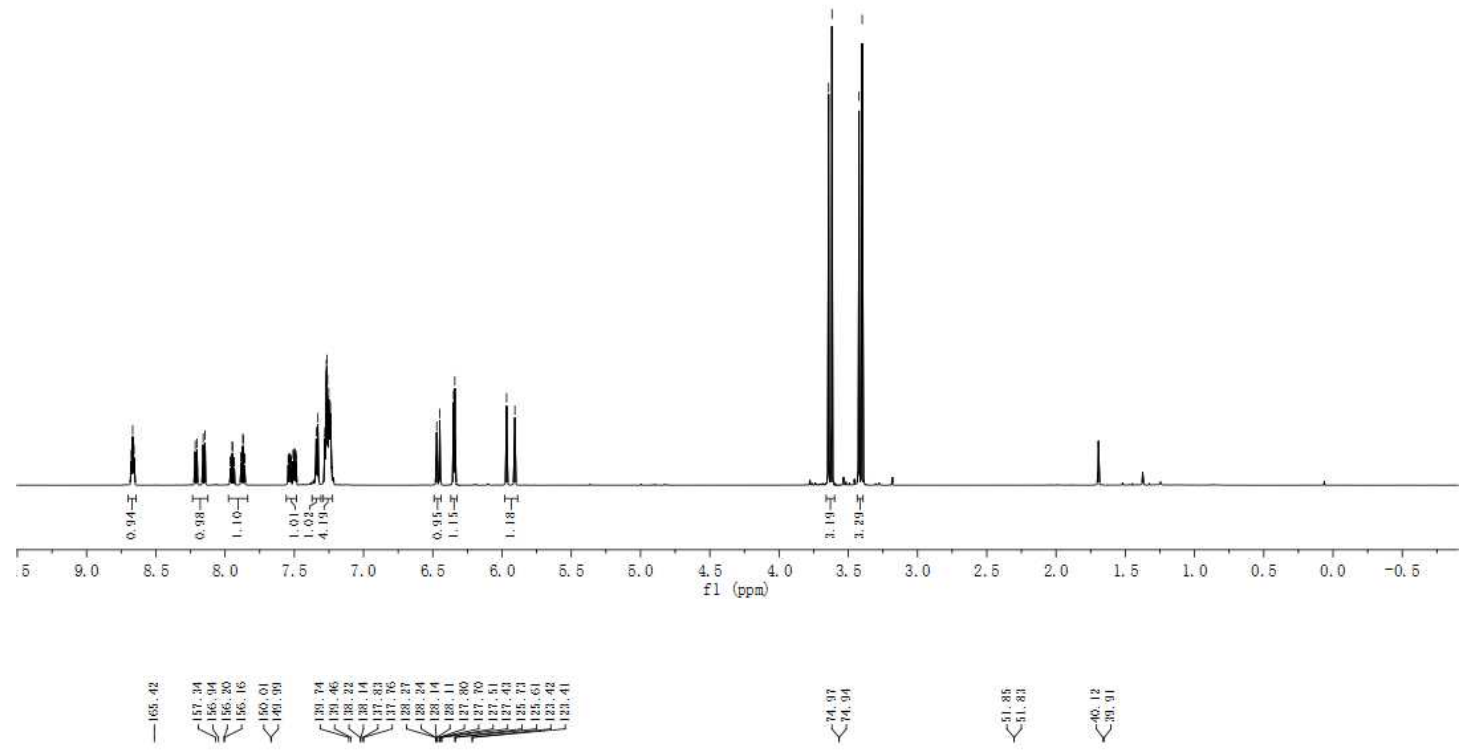

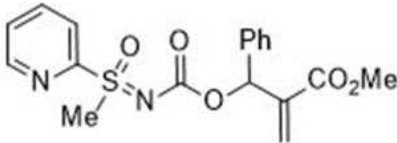

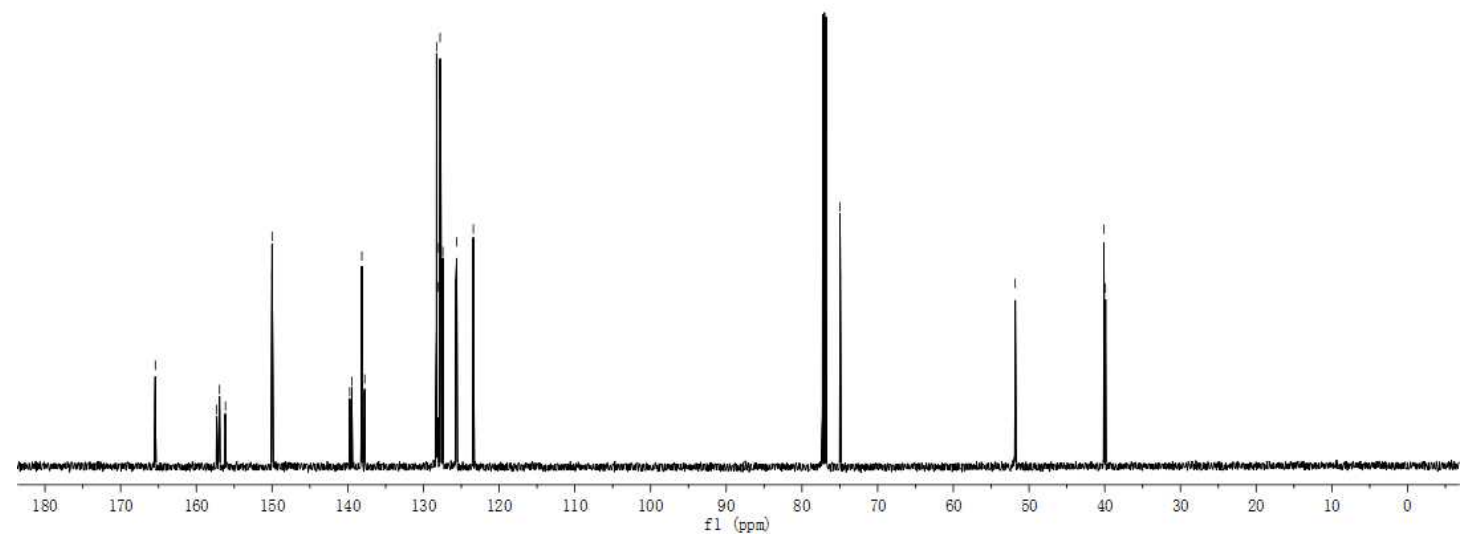


$N$-\{[(2-(Methoxycarbonyl)-1-phenylallyl)oxy]carbonyl $\}-S$-methyl-S-styrylsulfoximine (3ia)

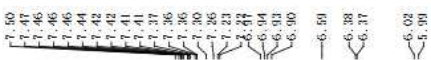<smiles>C=C(C(C)=O)C(OC(=O)N=S(C)(=O)/C=C/c1ccccc1)c1ccccc1</smiles>

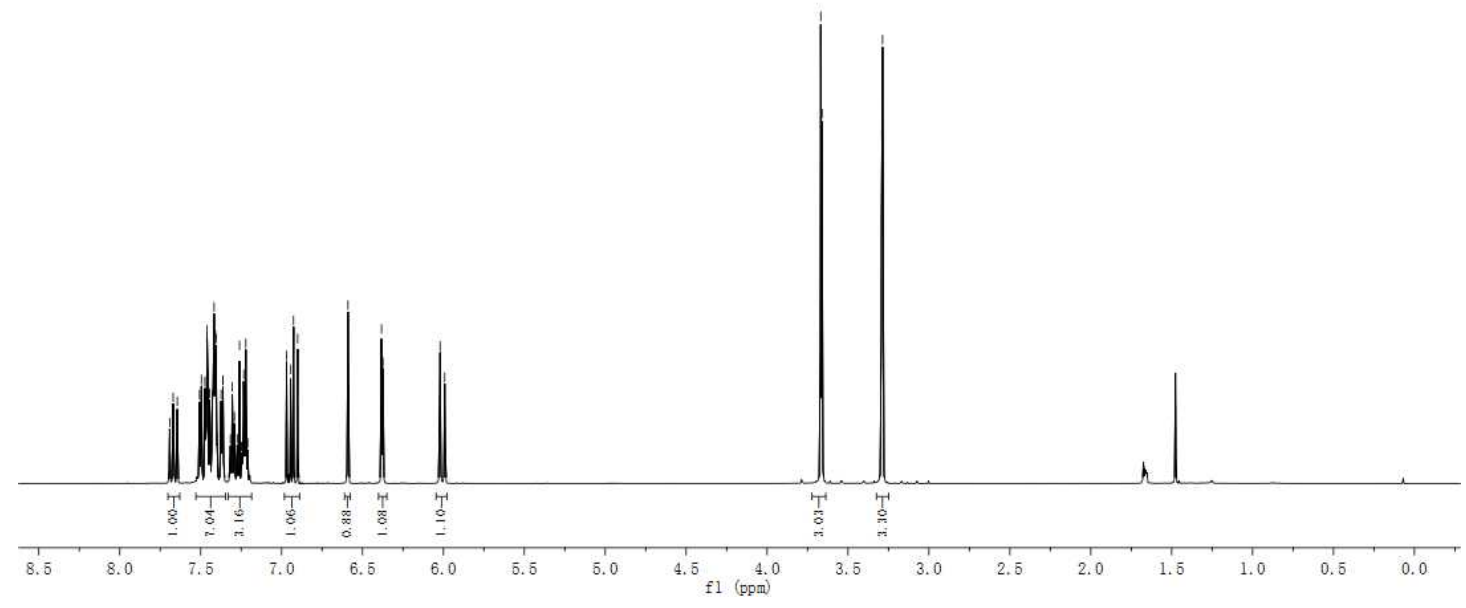

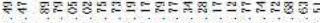

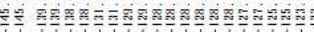

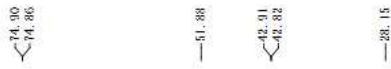<smiles>C=C(C(=O)OC(=O)N=S(C)(=O)/C=C/c1ccccc1)c1ccccc1</smiles>

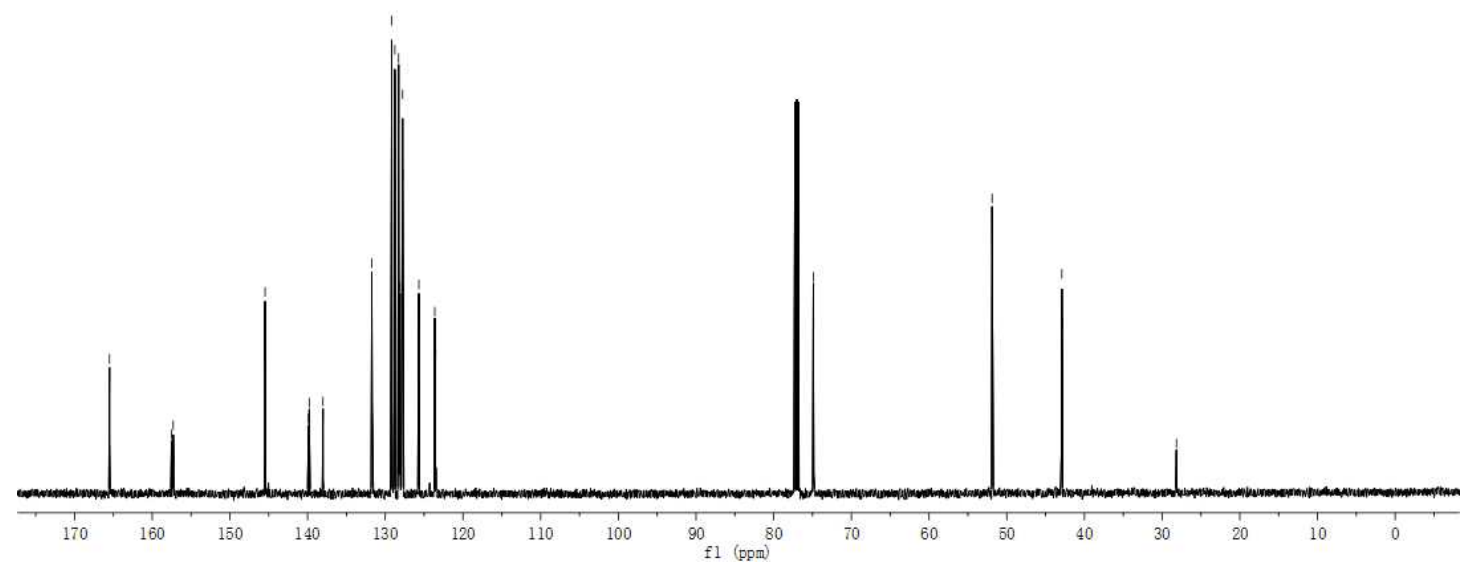


$N$-\{[(2-(methoxycarbonyl)-1-phenylallyl)oxy]carbonyl $\}-S$-methyl-S-(tert-butyl)sulfoximine (3ja)

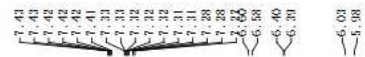

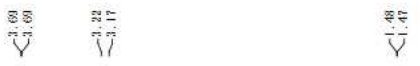<smiles>C=C(C(C)=O)C(OC(=O)N=S(C)(=O)C(C)(C)C)c1ccccc1</smiles>
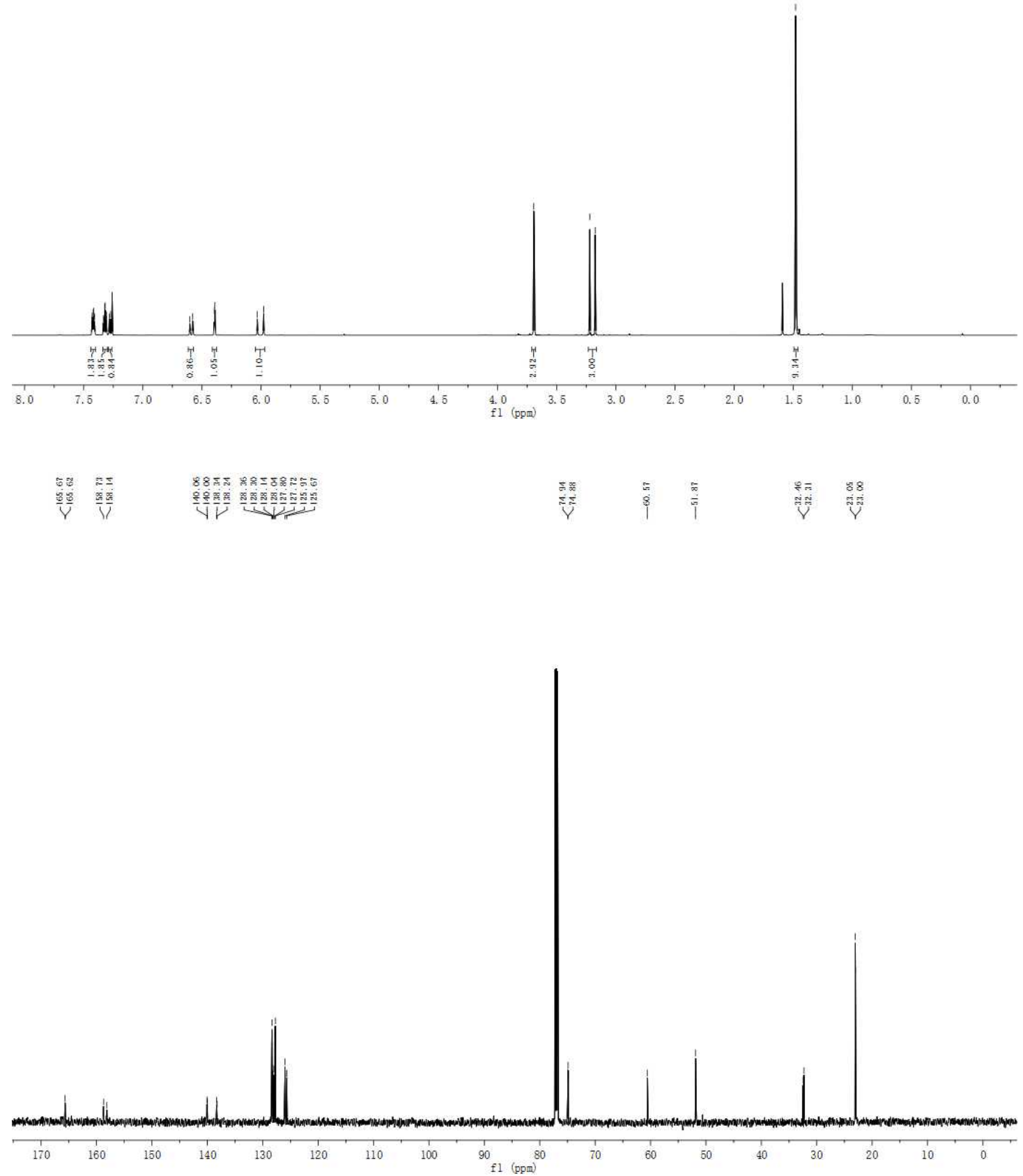
$N$-\{[(2-(Methoxycarbonyl)-1-phenylallyl)oxy]carbonyl $\}$-tetrahydrothiophenesulfoximine (3ka)

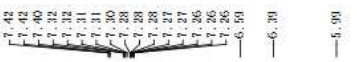

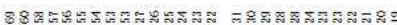

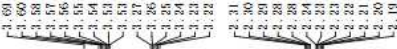

$\mathrm{S}_{\text {"in }}^{\mathrm{O}} \mathrm{N}_{\mathrm{O}}^{\mathrm{O}} \mathrm{CO}_{2} \mathrm{Me}$
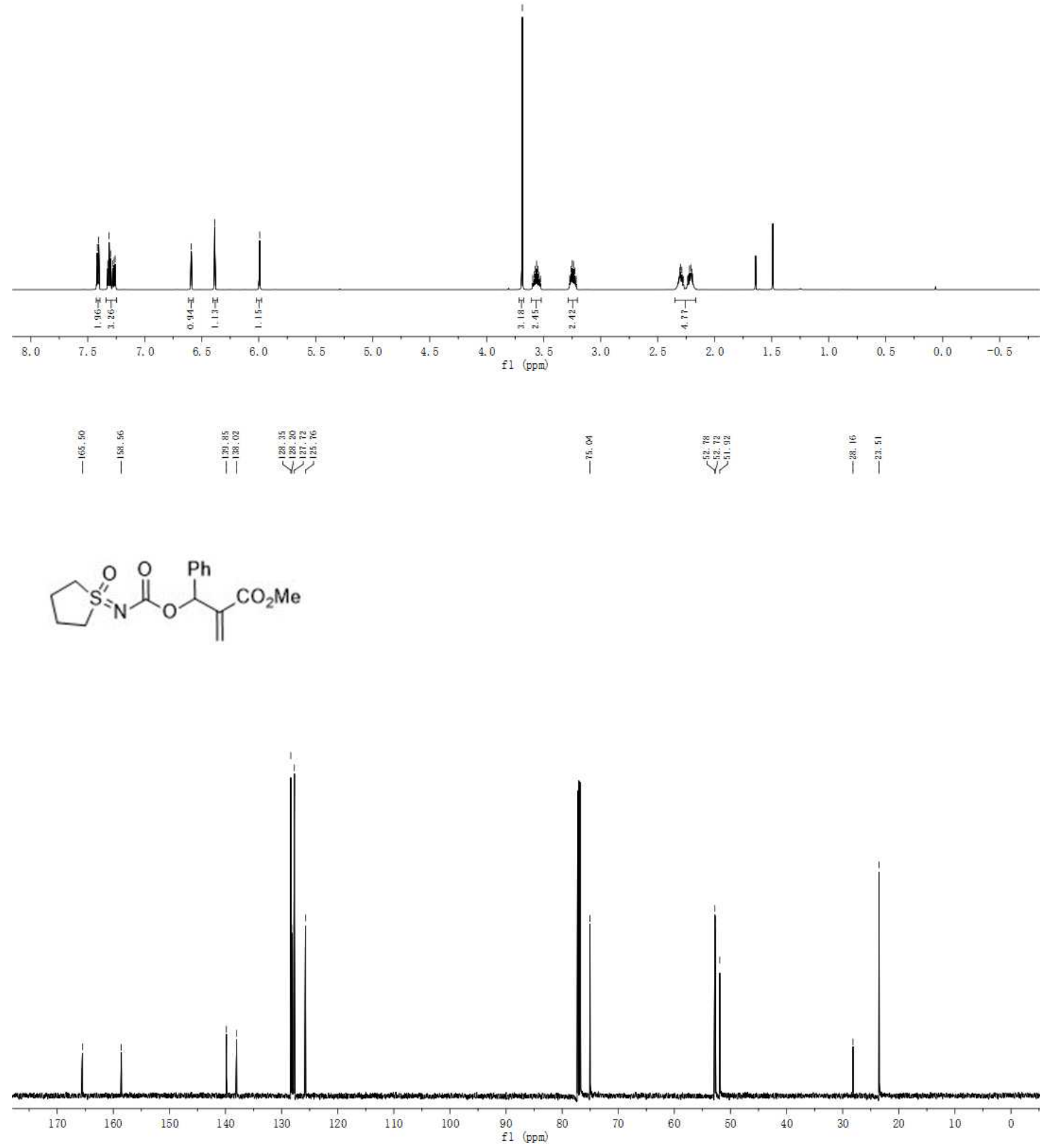
$N$-\{[(2-(Methoxycarbonyl)-1-phenylallyl)oxy]carbonyl $\}-S, S$-diphenylsulfoximine (3la)

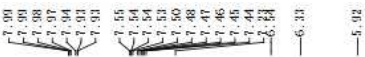

$\stackrel{5}{1}$<smiles>C=C(C(C)=O)C(OC(=O)N=S(=O)(c1ccccc1)c1ccccc1)c1ccccc1</smiles>

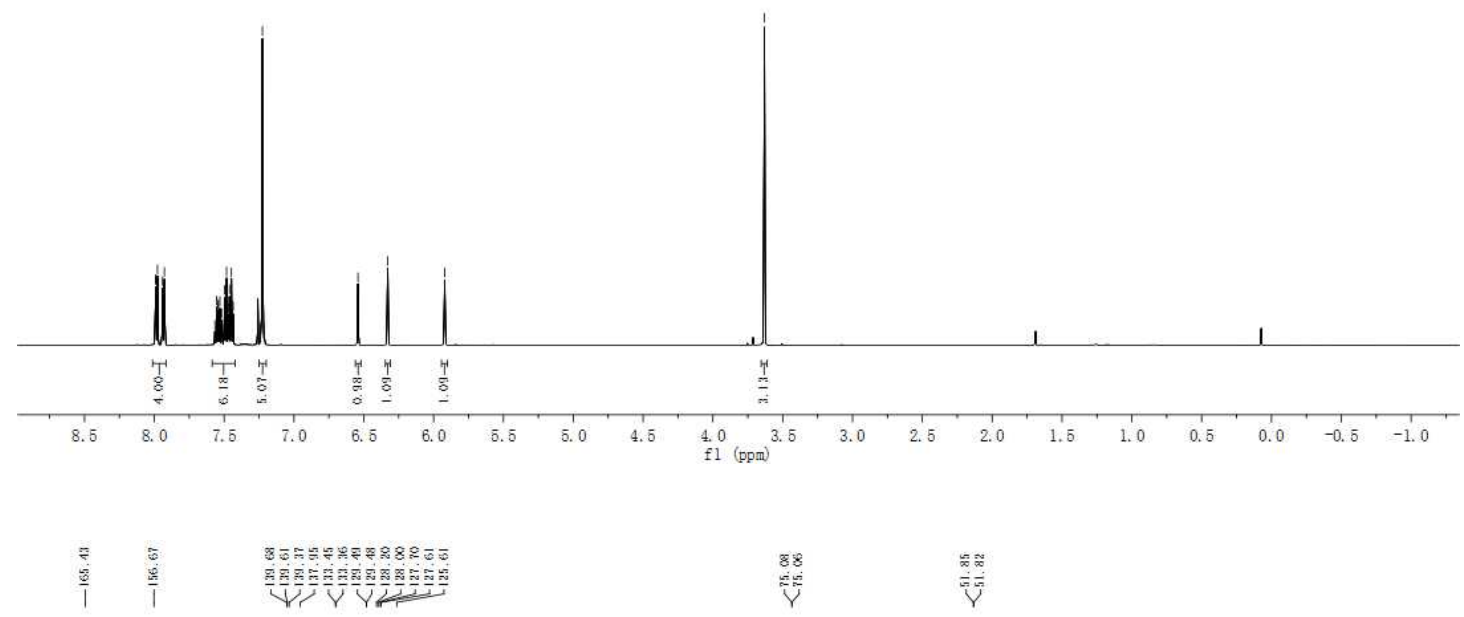

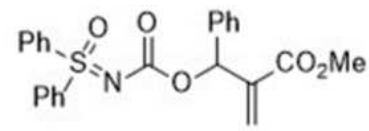

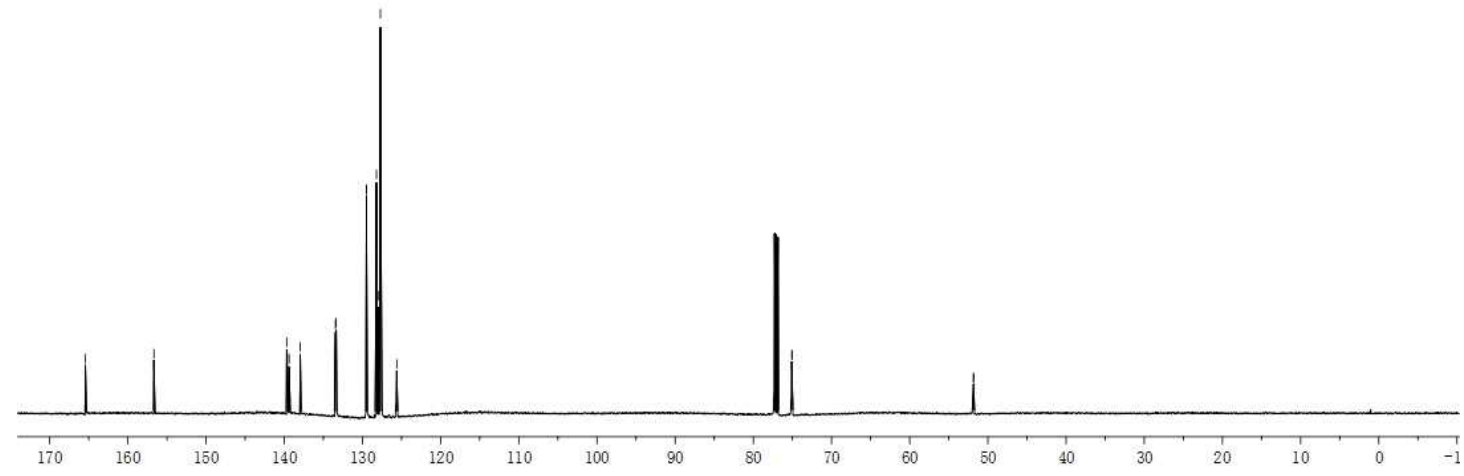


$N$-\{[(2-(Methoxycarbonyl)-1-(p-tolyl)allyl)oxy]carbonyl $\}-S$-methyl-S-phenylsulfoximine (3ab)

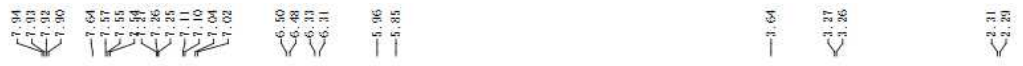<smiles>C=C(C(C)=O)C(OC(=O)N=S(C)(=O)c1ccccc1)c1ccc(C)cc1</smiles>

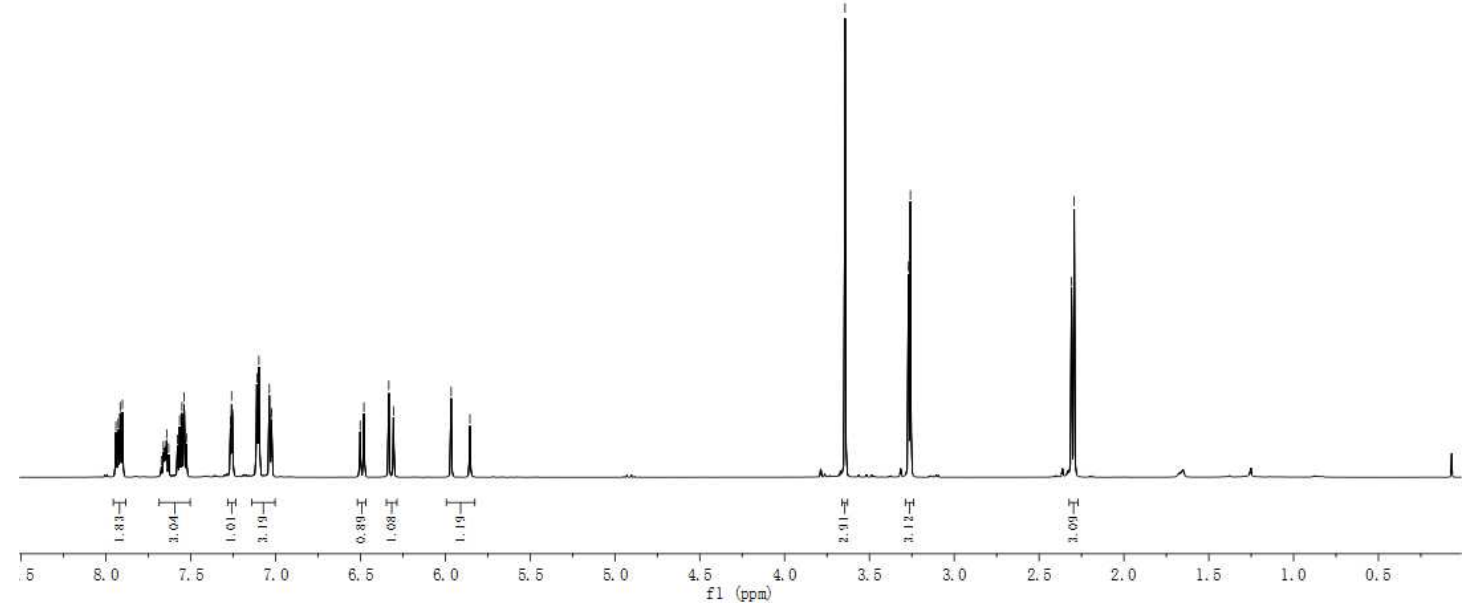

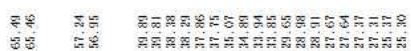

Y V

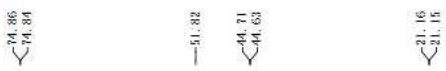
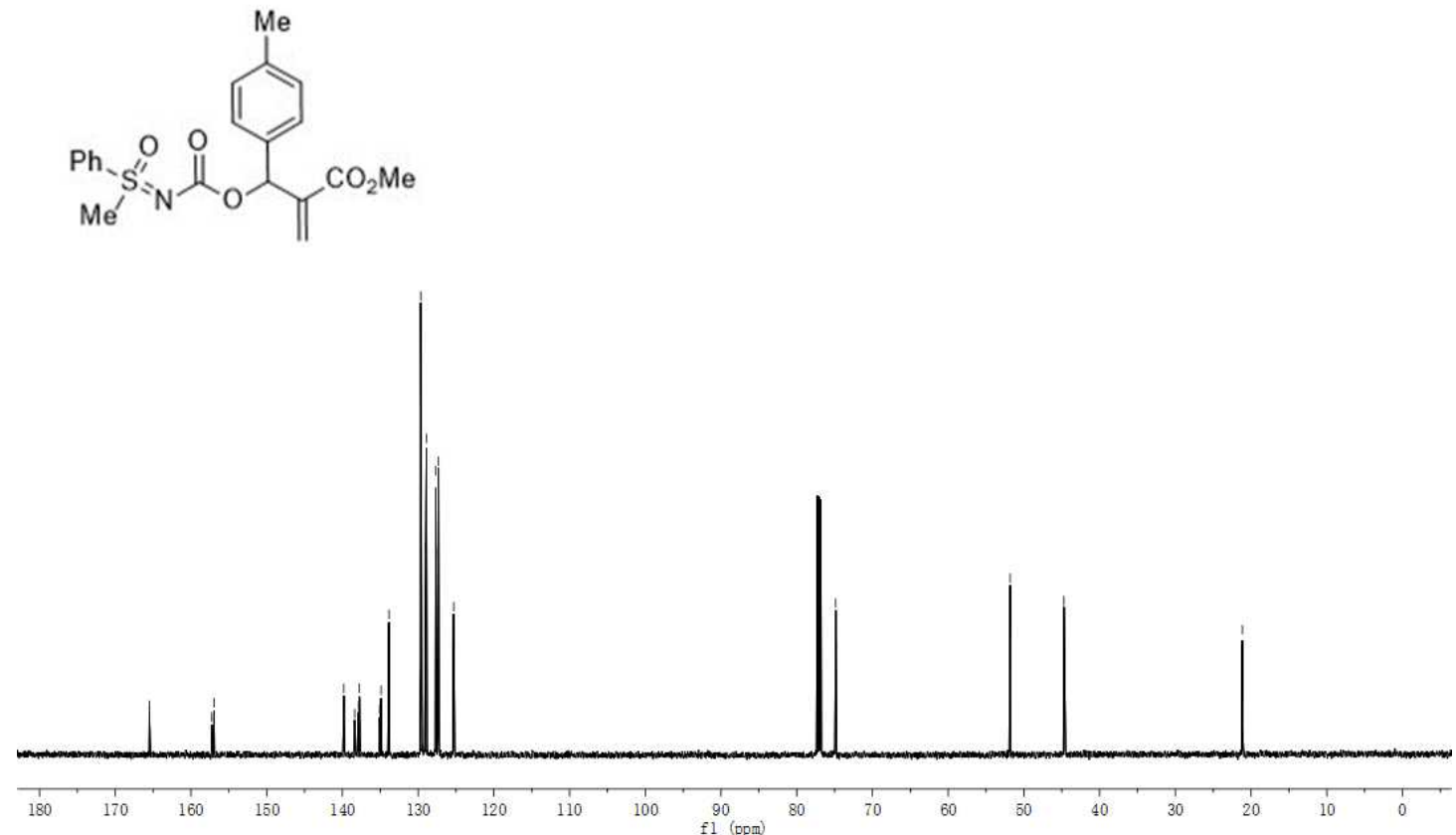
$N$-\{[(2-(Methoxycarbonyl)-1-(m-tolyl)allyl)oxy]carbonyl $\}-S$-methyl-S-phenylsulfoximine (3ac)

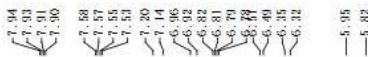<smiles>C=C(C(C)=O)C(OC(=O)N=S(C)(=O)c1ccccc1)c1cccc(C)c1</smiles>

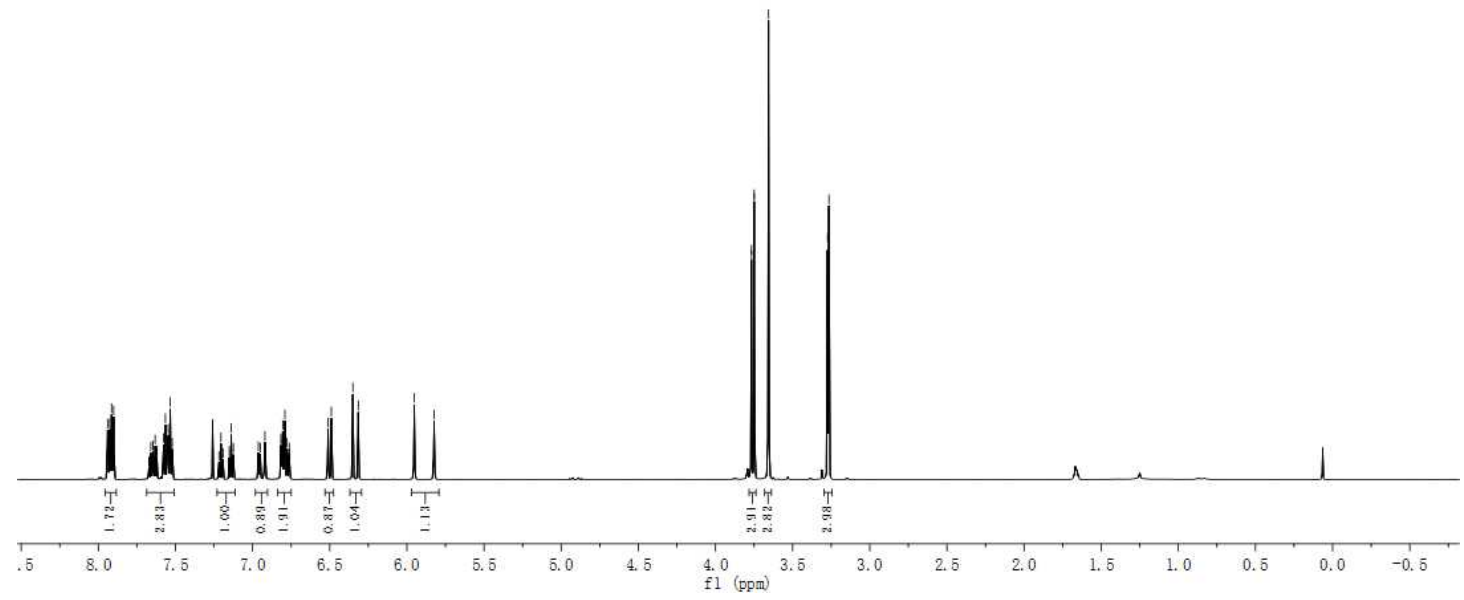

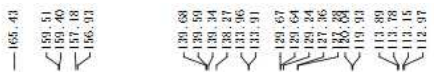

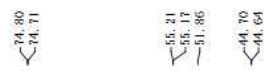
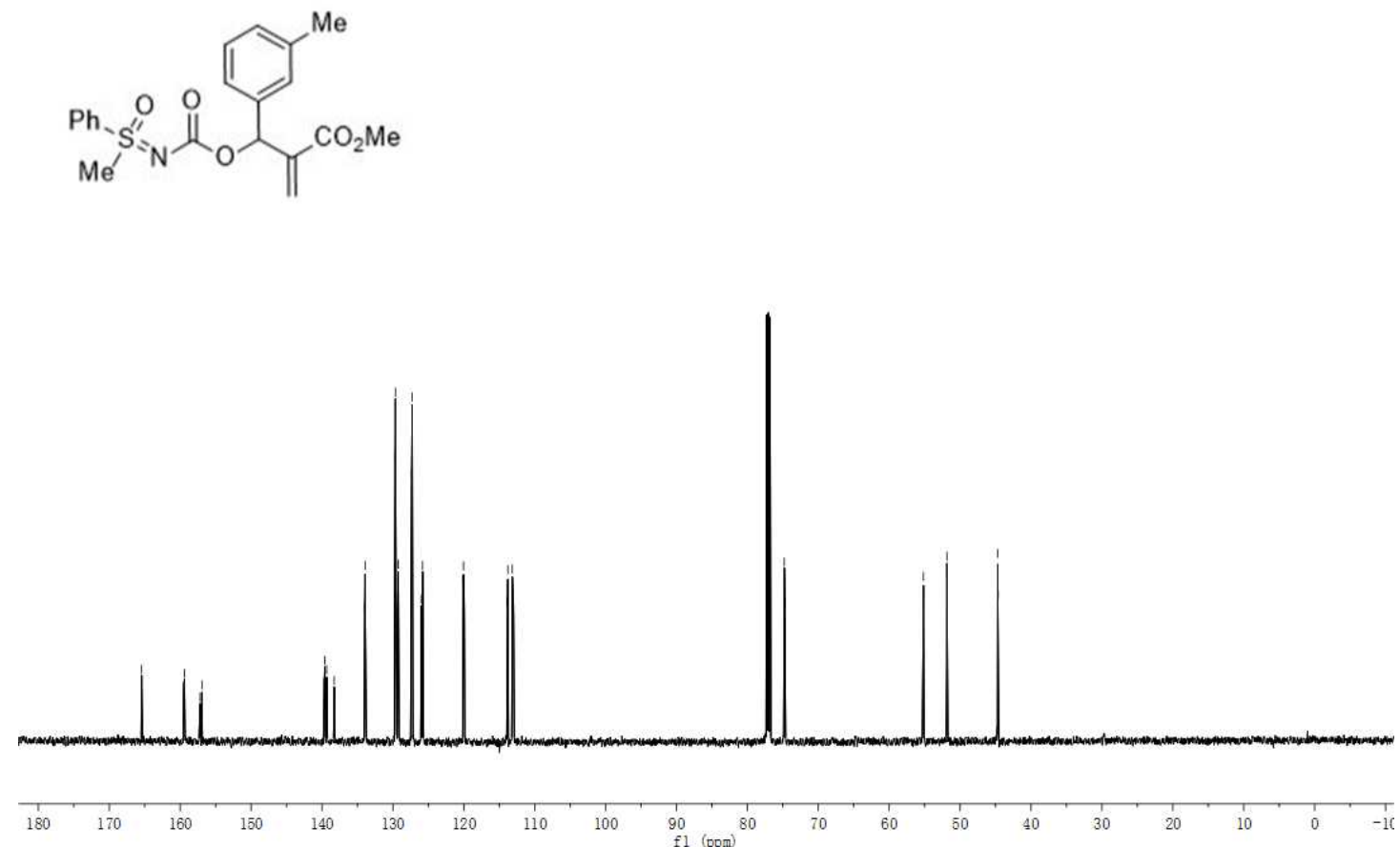
$N$-\{[(2-(Methoxycarbonyl)-1-[4-(methylthio)phenyl]allyl)oxy]carbonyl $\}-S$-methyl-S-phenylsulfoximine (3ad)

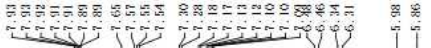

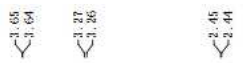<smiles>C=C(C(C)=O)C(OC(=O)NS(C)(=O)=O)c1ccc(C)cc1</smiles>

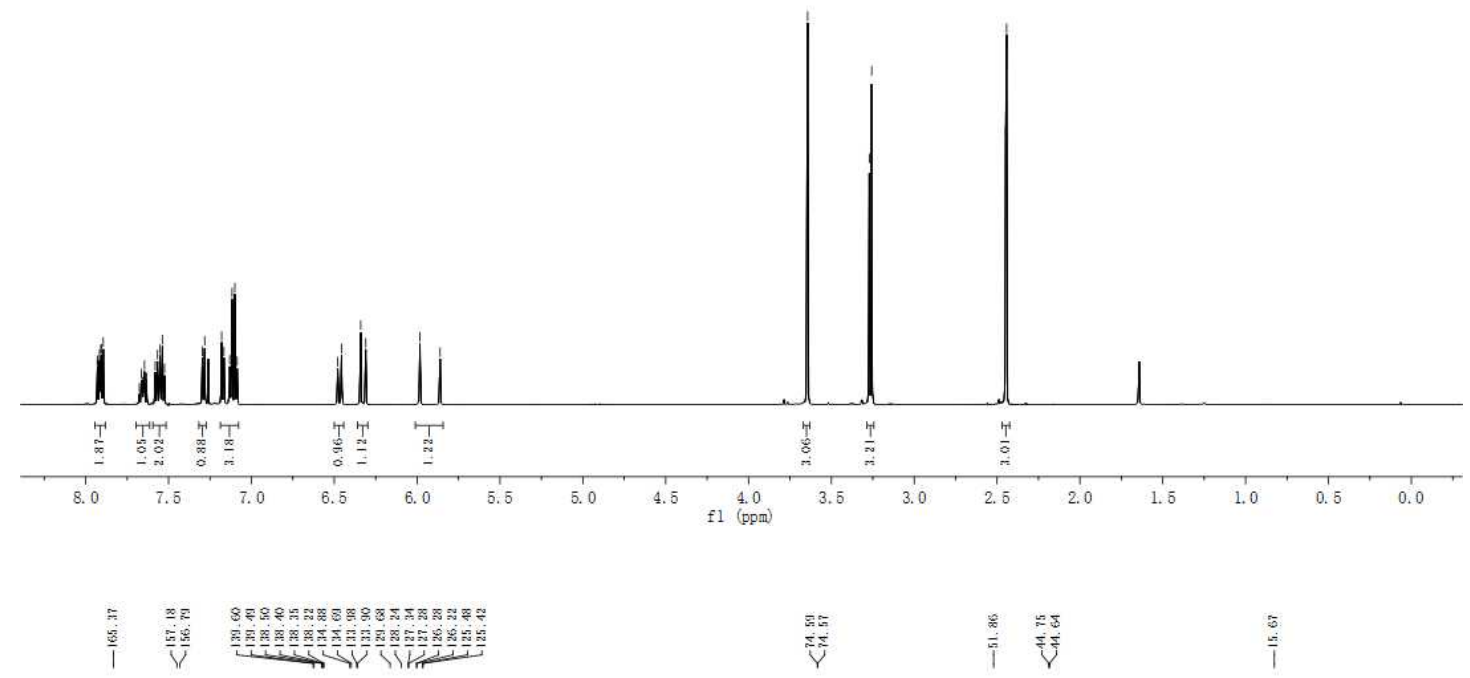<smiles>C=C(C(C)=O)C(OC(=O)N=S(C)(=O)c1ccc(C)cc1)c1ccc(C)cc1</smiles>

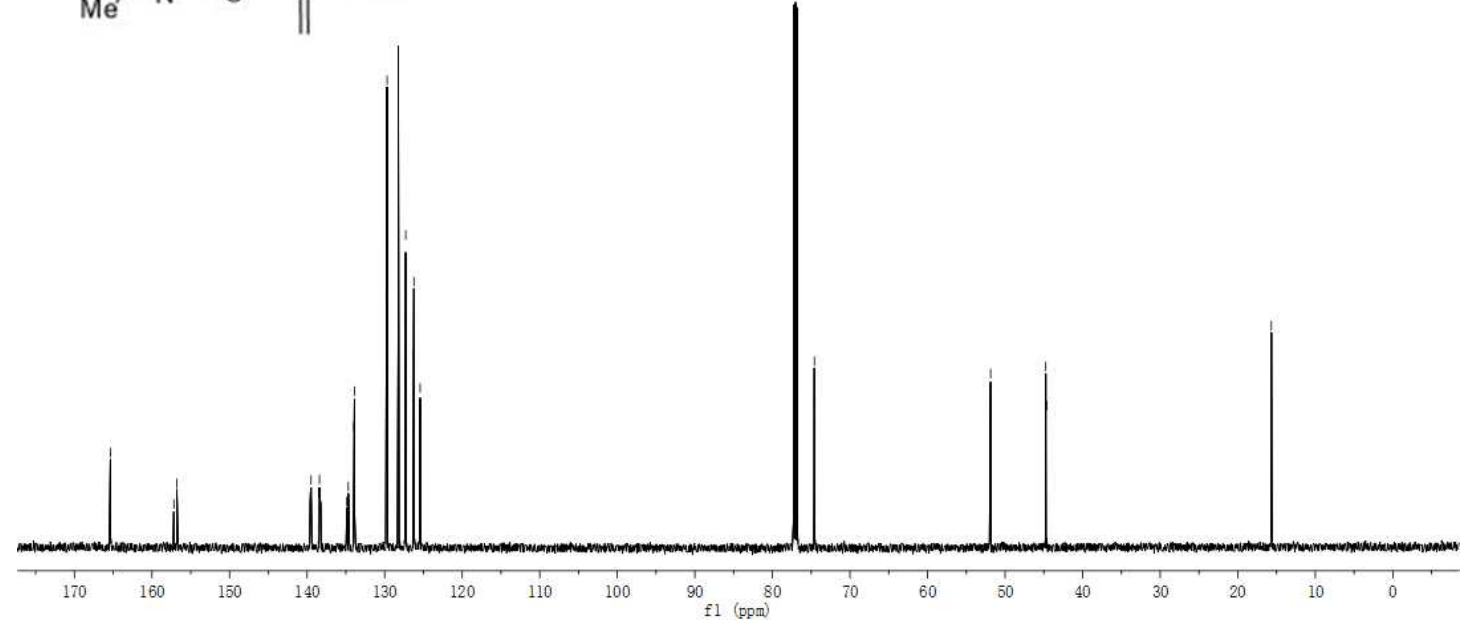


$N$-\{[(2-(Methoxycarbonyl)-1-(4-bromophenyl)allyl)oxy]carbonyl $\}-S$-methyl-S-phenylsulfoximine (3ae)

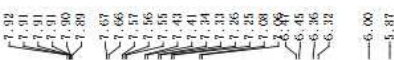

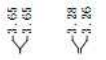<smiles>C=C(C(C)C)C(OC)OC(=O)N=S(C)(=O)c1ccccc1</smiles>

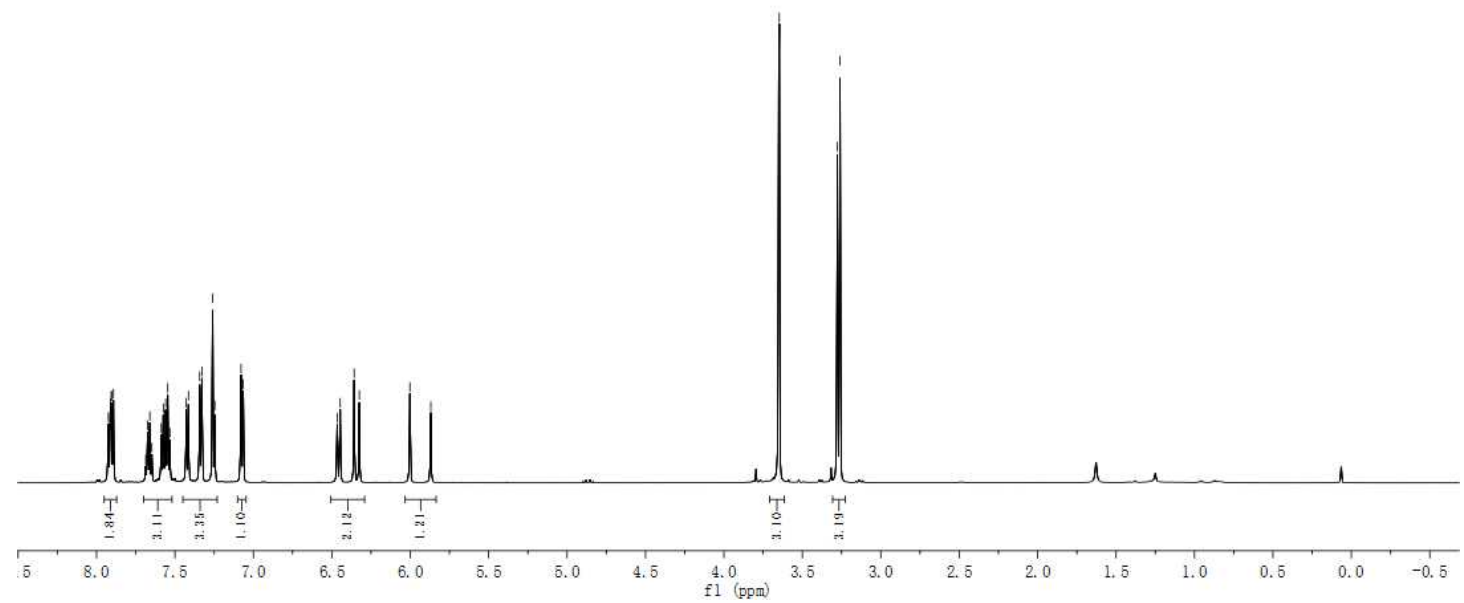

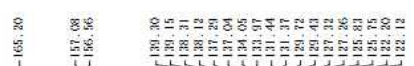

ISTV V

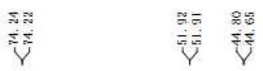<smiles>C=C(C(OC)OC(=O)N=S(C)(=O)c1ccccc1)C(C(=O)OC)c1ccc(Br)cc1</smiles>

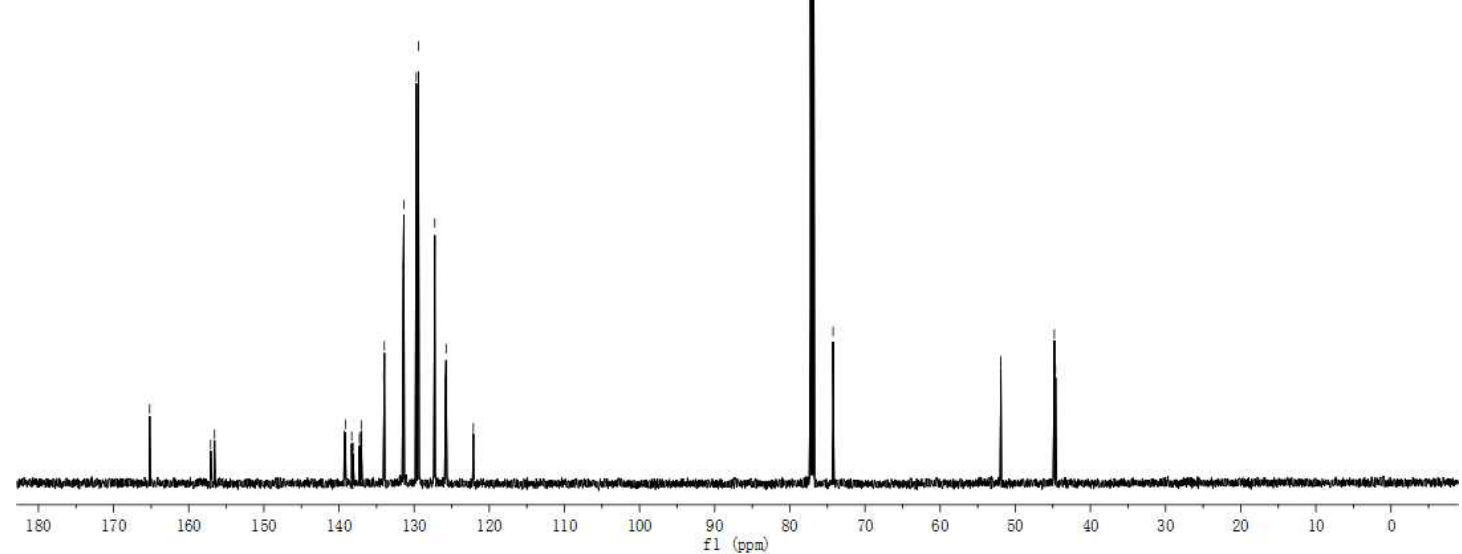


$N$-\{[(2-(Methoxycarbonyl)-1-(3-bromophenyl)allyl)oxy]carbonyl $\}-S$-methyl-S-phenylsulfoximine (3af)

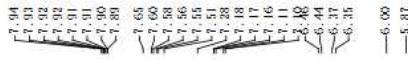

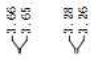<smiles>C=C(OC(=O)N=S(C)(=O)c1ccccc1)C(C(C)=O)c1cccc(Br)c1</smiles>

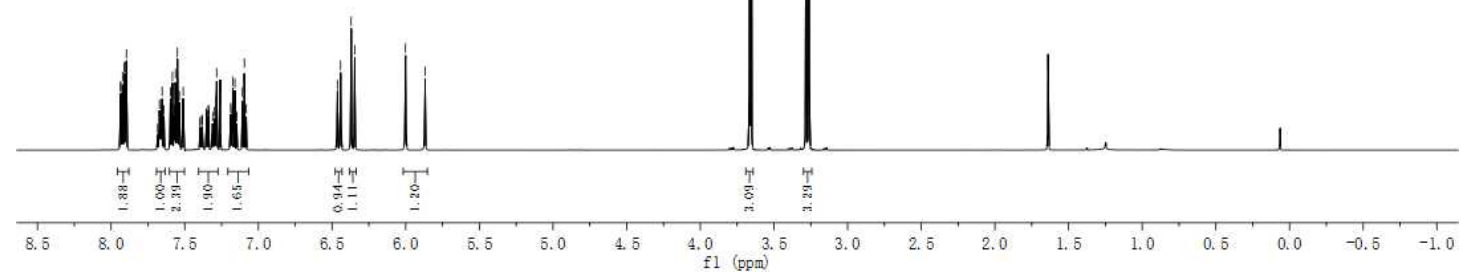

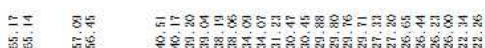

Y

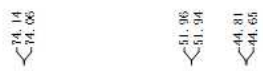
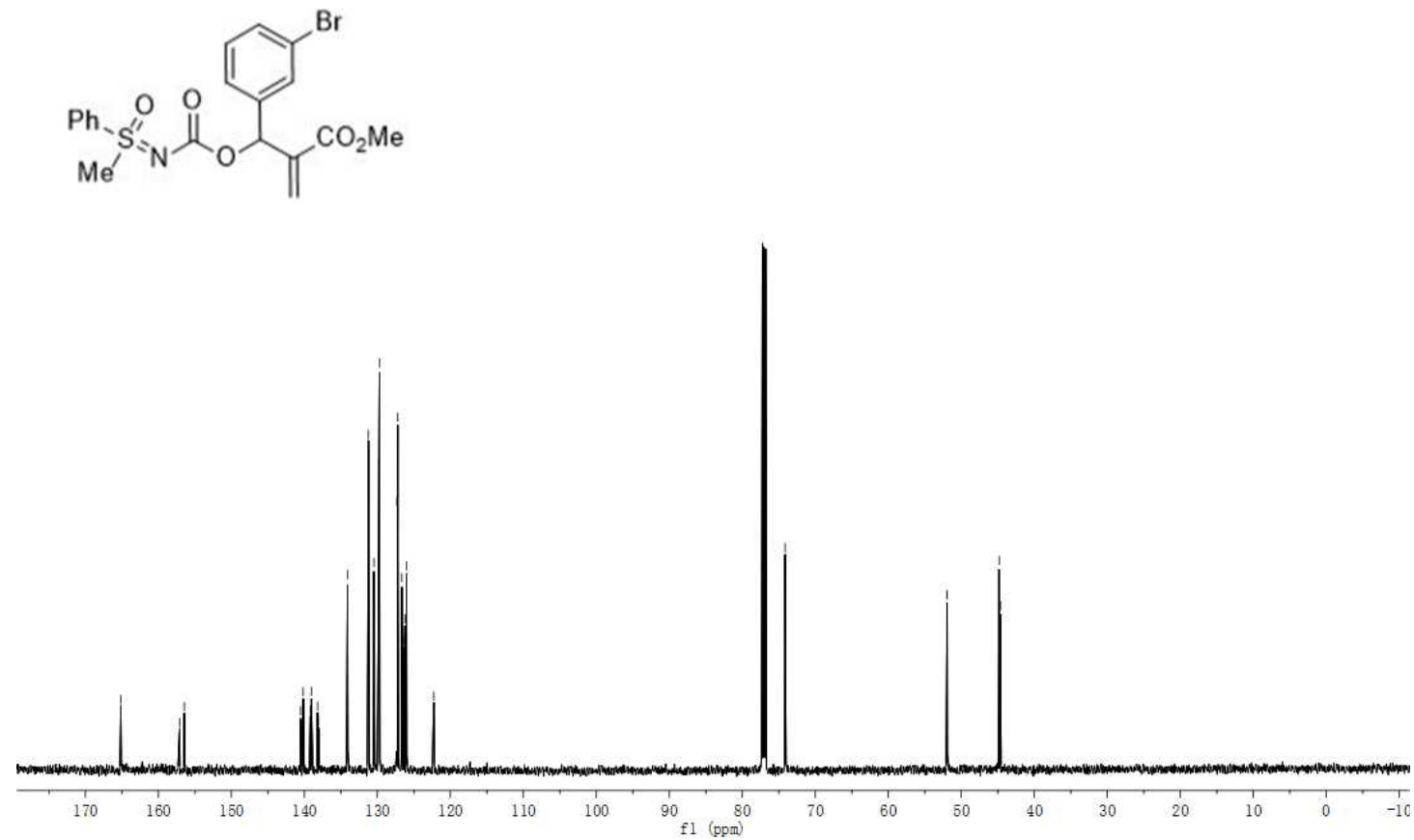
$N$-\{[(2-(Methoxycarbonyl)-1-(2-bromophenyl)allyl)oxy $]$ carbonyl $\}-S$-methyl-S-phenylsulfoximine (3ag)

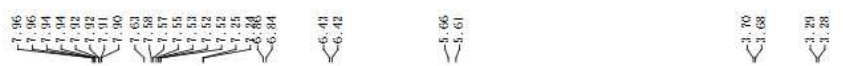

$$
\text { } \mathrm{Me}^{\mathrm{s}_{\mathrm{N}}}
$$
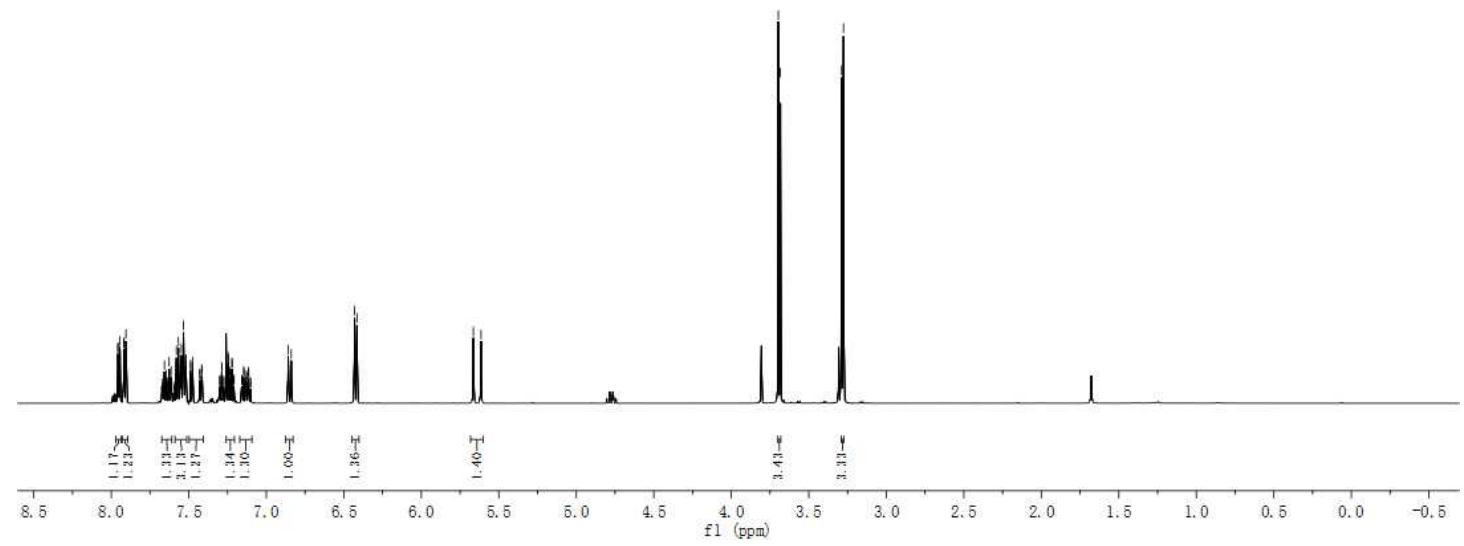

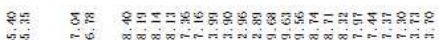

$Y$ V
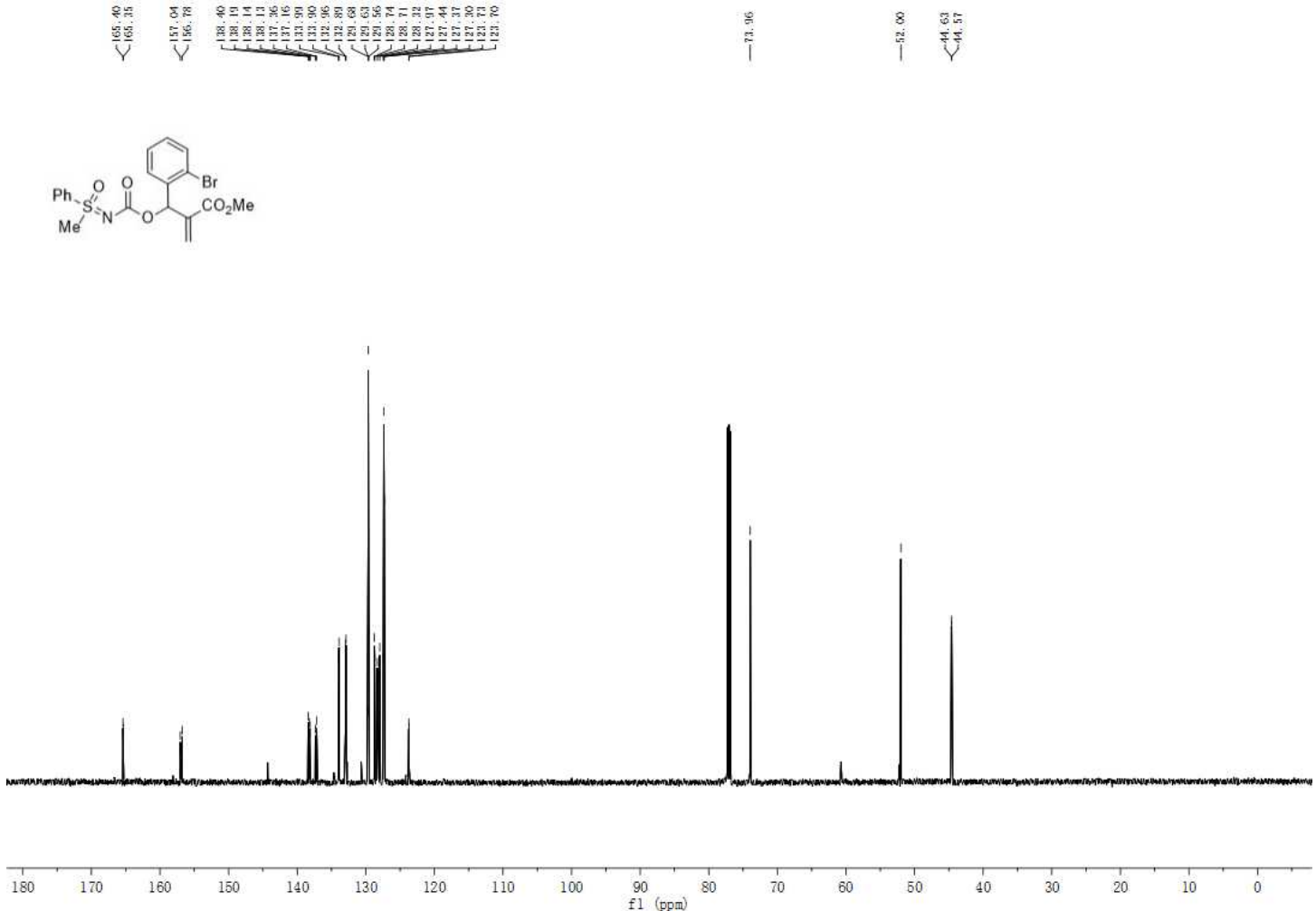
$N$-\{[(2-(Methoxycarbonyl)-1-(4-nitrophenyl)allyl)oxy]carbonyl $\}-S$-methyl-S-phenylsulfoximine (3ah)

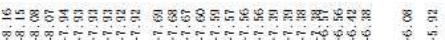

$\stackrel{5}{i}$<smiles>C=C(C(C)=O)C(OC(=O)N=S(C)(=O)c1ccccc1)c1ccc([N+](=O)[O-])cc1</smiles>

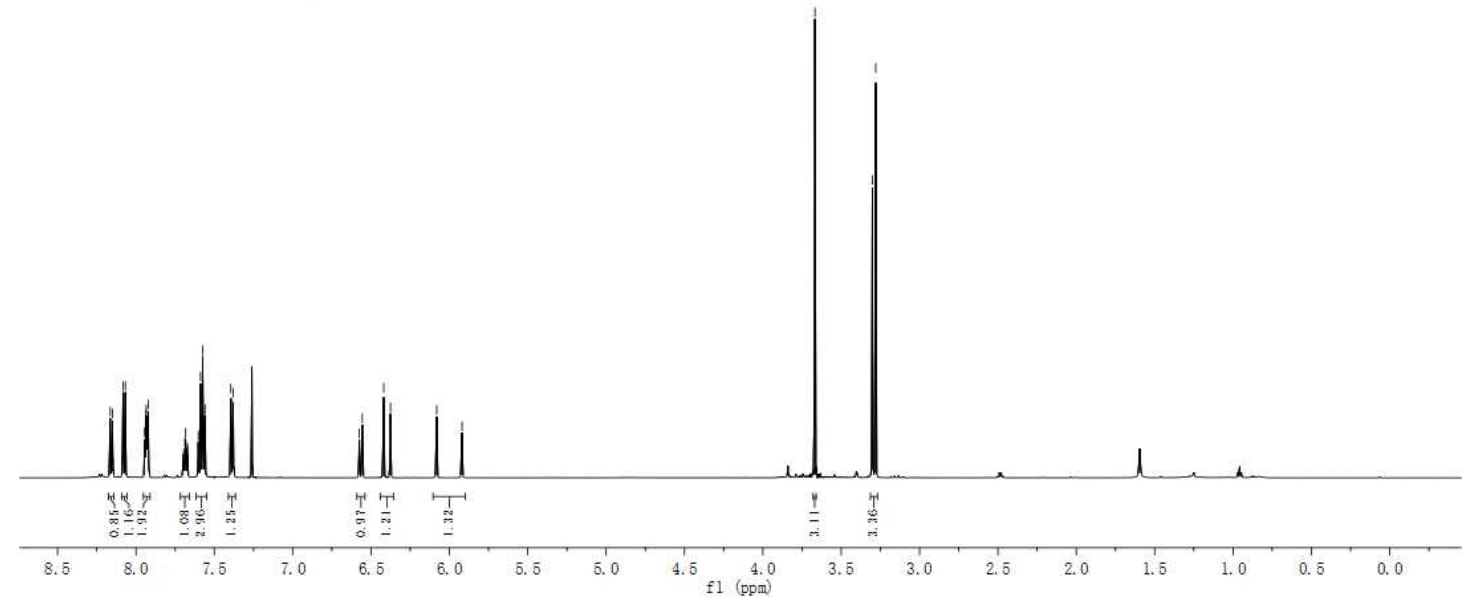

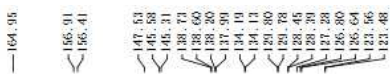

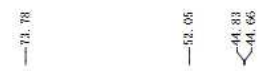<smiles>C=C(C(C)=O)C(OC(=O)N=S(C)(=O)c1ccccc1)c1ccc([N+](=O)[O-])cc1</smiles>

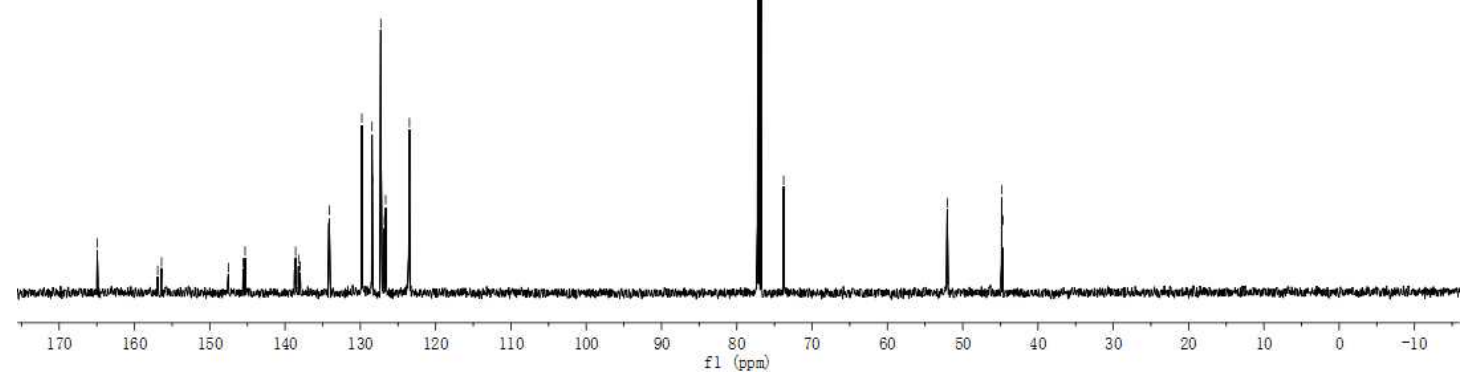


$N$-\{[(2-(Methoxycarbonyl)-1-[4-(trifluoromethyl)phenyl]allyl)oxy]carbonyl $\}-S$-methyl-S-phenylsulfoximine (3ai)

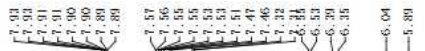

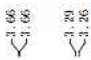<smiles>C=C(OC)C(OC(=O)N=S(C)(=O)c1ccccc1)c1ccc(C(F)(F)F)cc1</smiles>

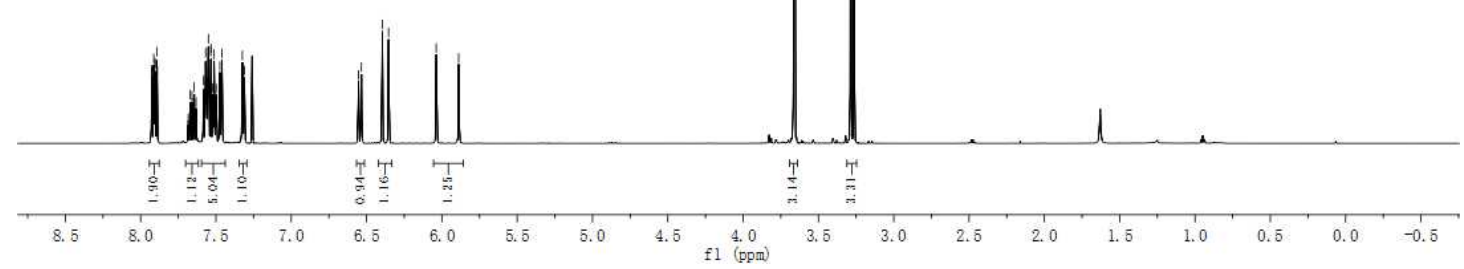

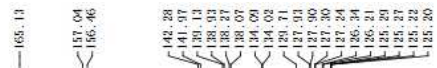

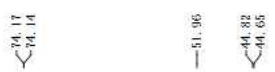<smiles>C=C(C(OC)OC(=O)N=S(C)(=O)c1ccccc1)C(C(C)=O)c1ccc(C(F)(F)F)cc1</smiles>

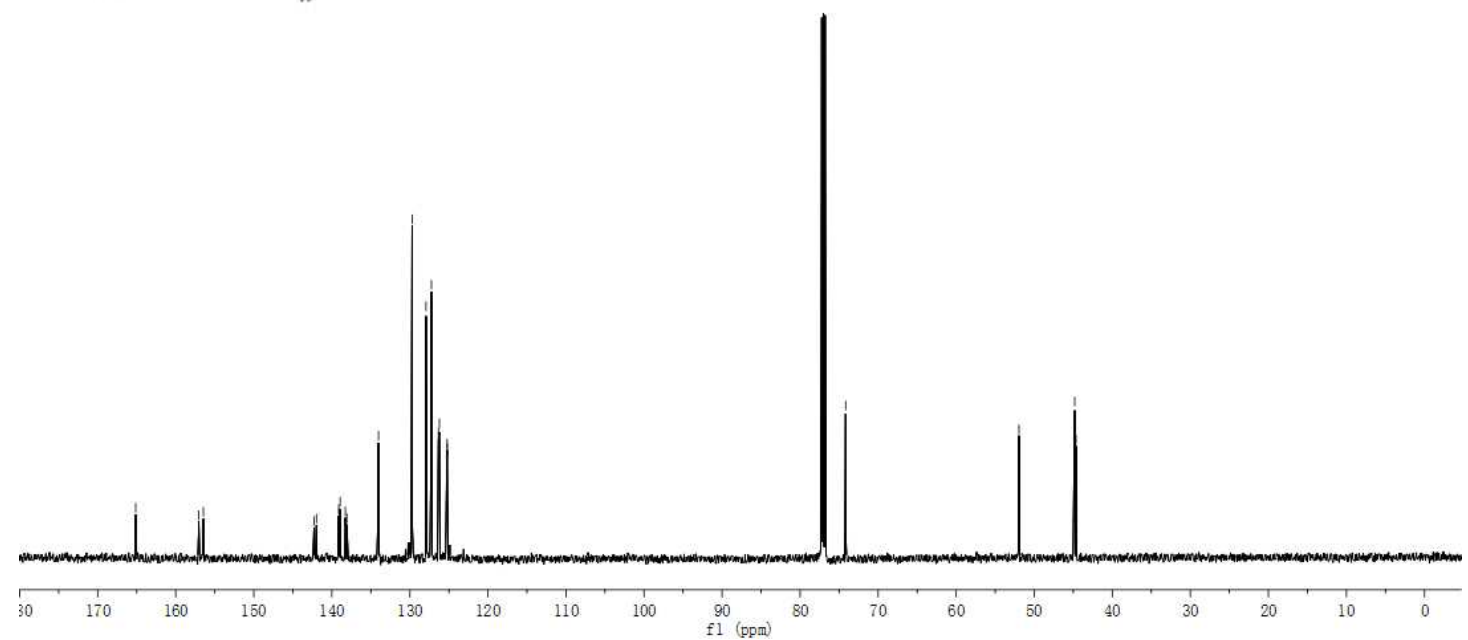


$N$-\{[(2-(Methoxycarbonyl)-1-(naphthalen-1-yl)allyl)oxy]carbonyl $\}-S$-methyl-S-phenylsulfoximine (3aj)

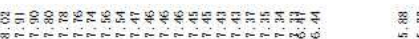

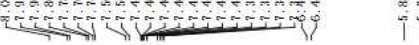

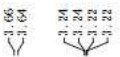<smiles>C=C(OC)C(OC(=O)N=S(C)(=O)c1ccccc1)c1cccc2ccccc12</smiles>

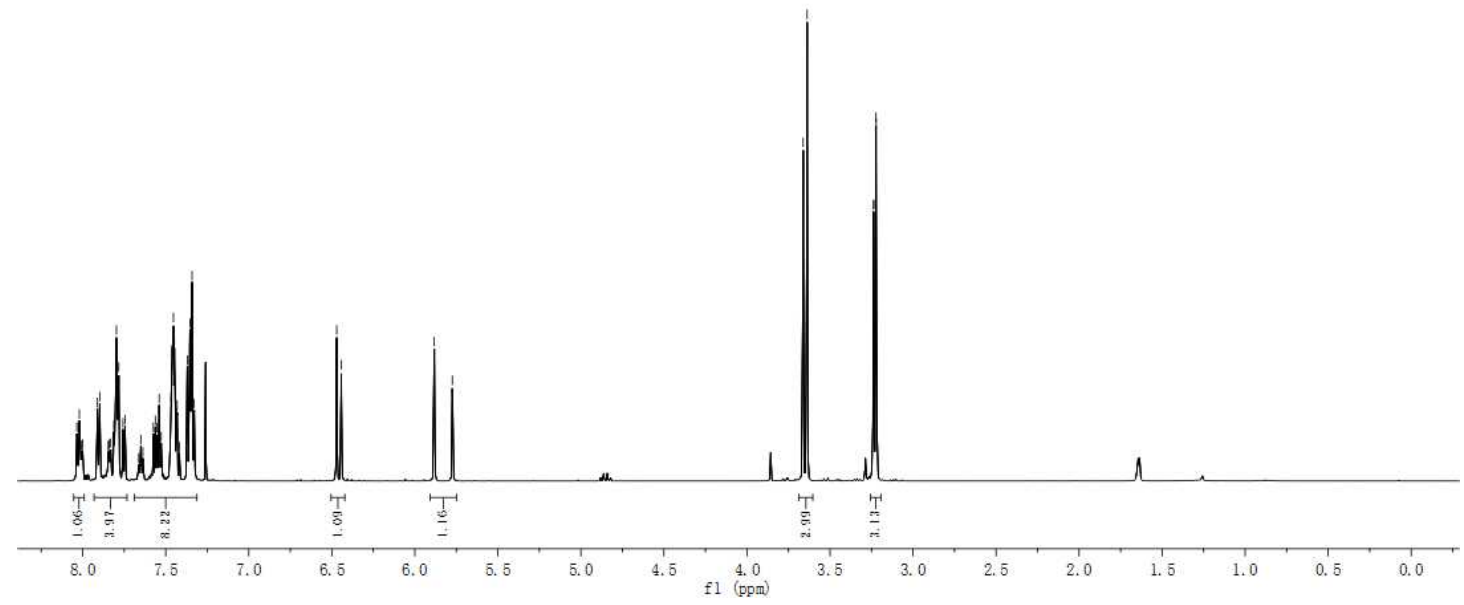

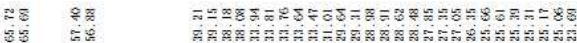

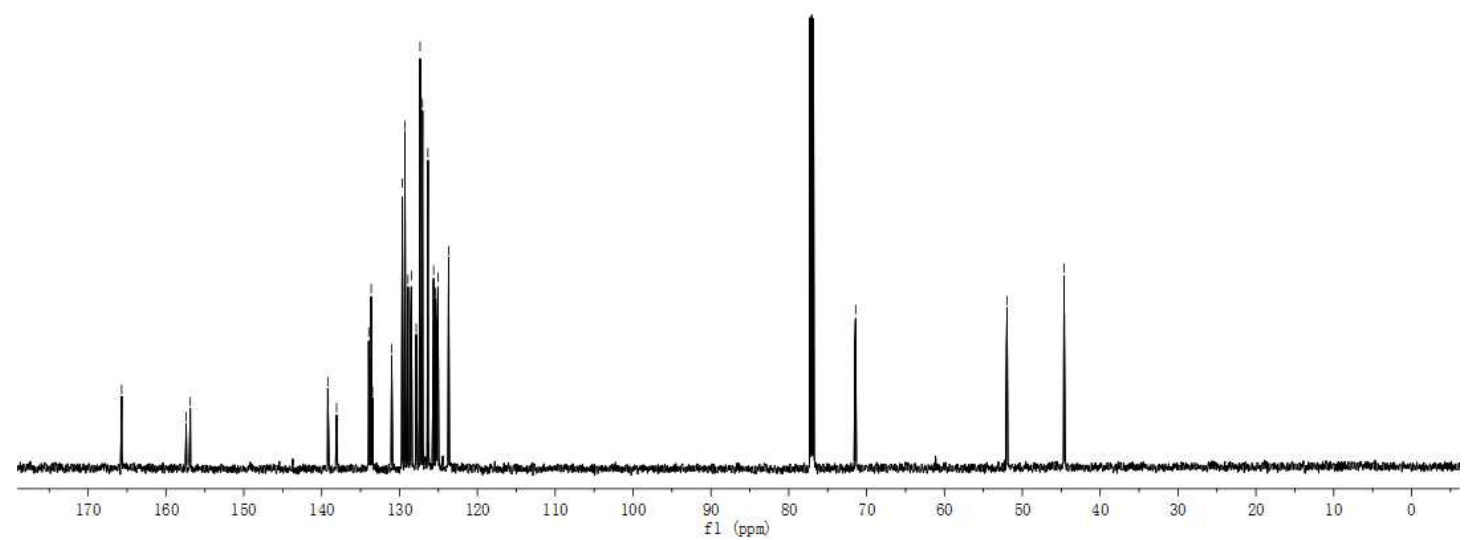


$N$-\{[(2-(Ethoxycarbonyl)-1-phenylallyl)oxy]carbonyl $\}-S$-methyl-S-phenylsulfoximine (3ak)

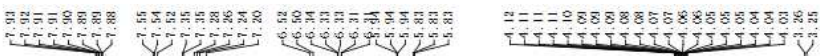

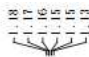

$\mathrm{Me}^{\prime} \mathrm{S}_{\mathrm{N}}^{\prime \prime}$

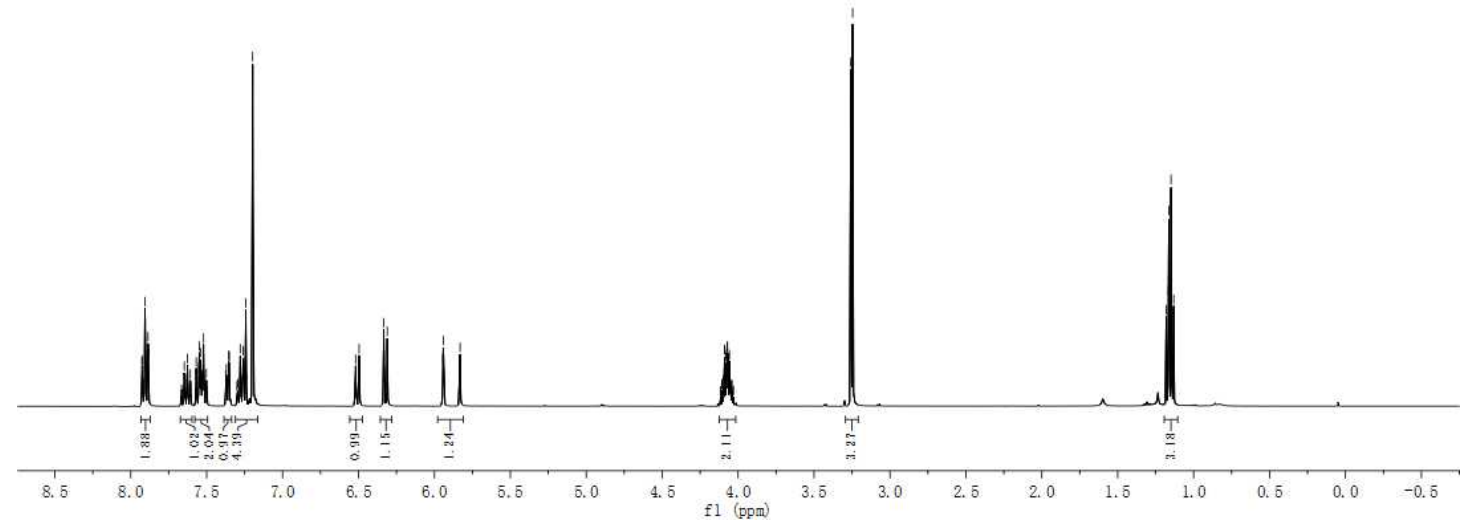

S8. $\quad$ \%

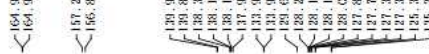

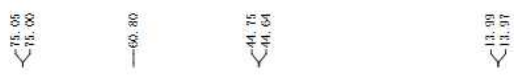<smiles>C=C(C(=O)N=S(C)(=O)c1ccccc1)C(=O)OC(c1ccccc1)c1ccccc1</smiles>

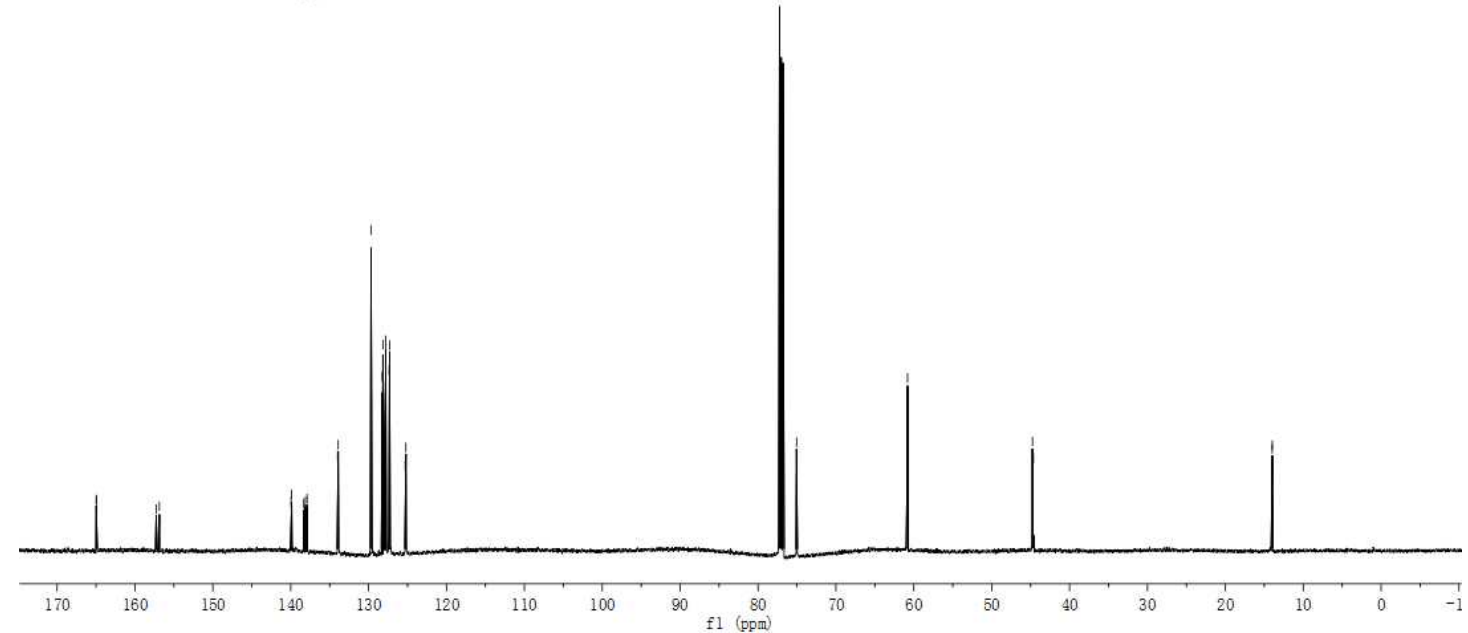


$N$-\{[(2-(Benzyloxycarbonyl)-1-phenylallyl)oxy]carbonyl $\}-S$-methyl-S-phenylsulfoximine (3al)

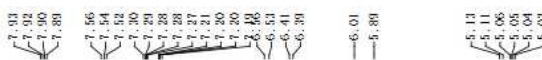

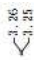<smiles>C=C(C(=O)OCc1ccccc1)C(OC(=O)N=S(C)(=O)c1ccccc1)c1ccccc1</smiles>

$\|d\|$
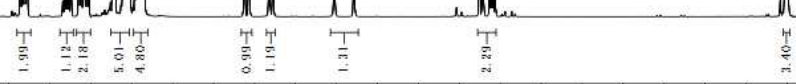

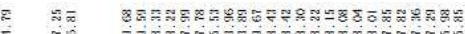

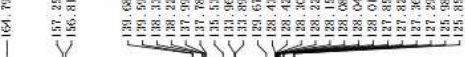

का 1815

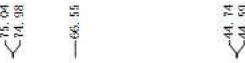<smiles>C=C(OCc1ccccc1)C(OC(=O)N=S(C)(=O)c1ccccc1)c1ccccc1</smiles>

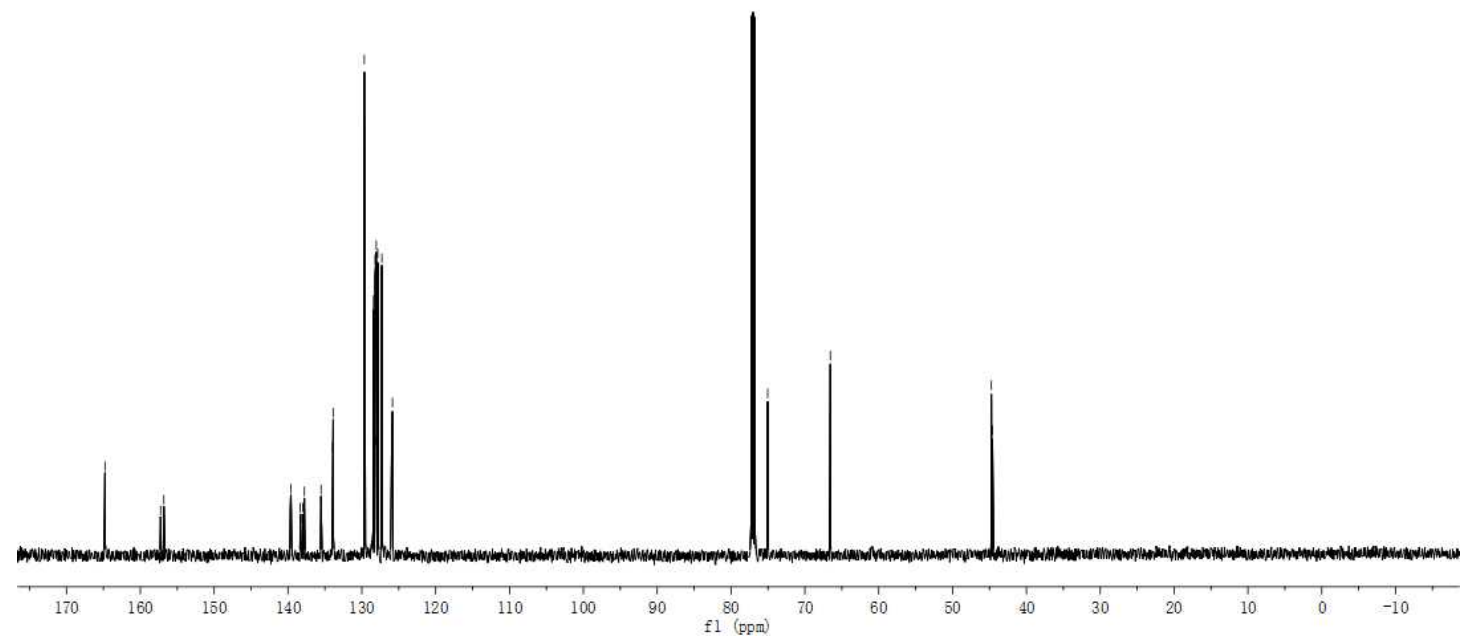


$N$-\{[(2-(Methoxycarbonyl)-1-(cyclohexyl)allyl)oxy]carbonyl $\}-S, S$-diphenylsulfoximine (3Im)

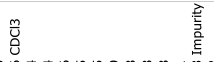

\% (O)
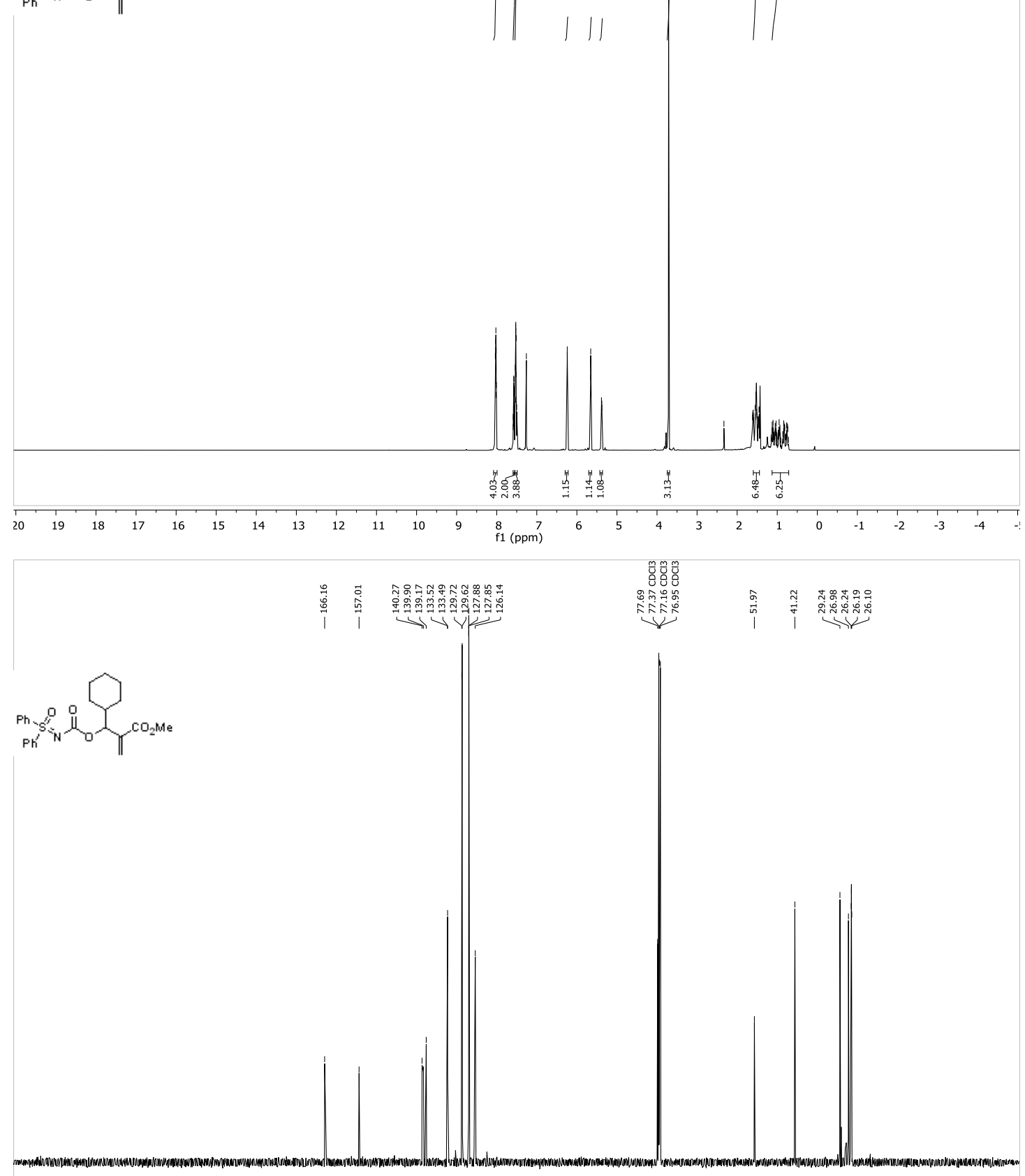

$\begin{array}{rllllllllllllllllllllllllllllll}1 & 240 & 230 & 220 & 210 & 200 & 190 & 180 & 170 & 160 & 150 & 140 & 130 & \underset{\mathrm{f} 1}{120} & 110 & 100 & 90 & 80 & 70 & 60 & 50 & 40 & 30 & 20 & 10 & 0 & -10\end{array}$ 
(E)-Imino(methyl)(styryl)- $\lambda^{6}$-sulfanone (1i)

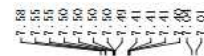
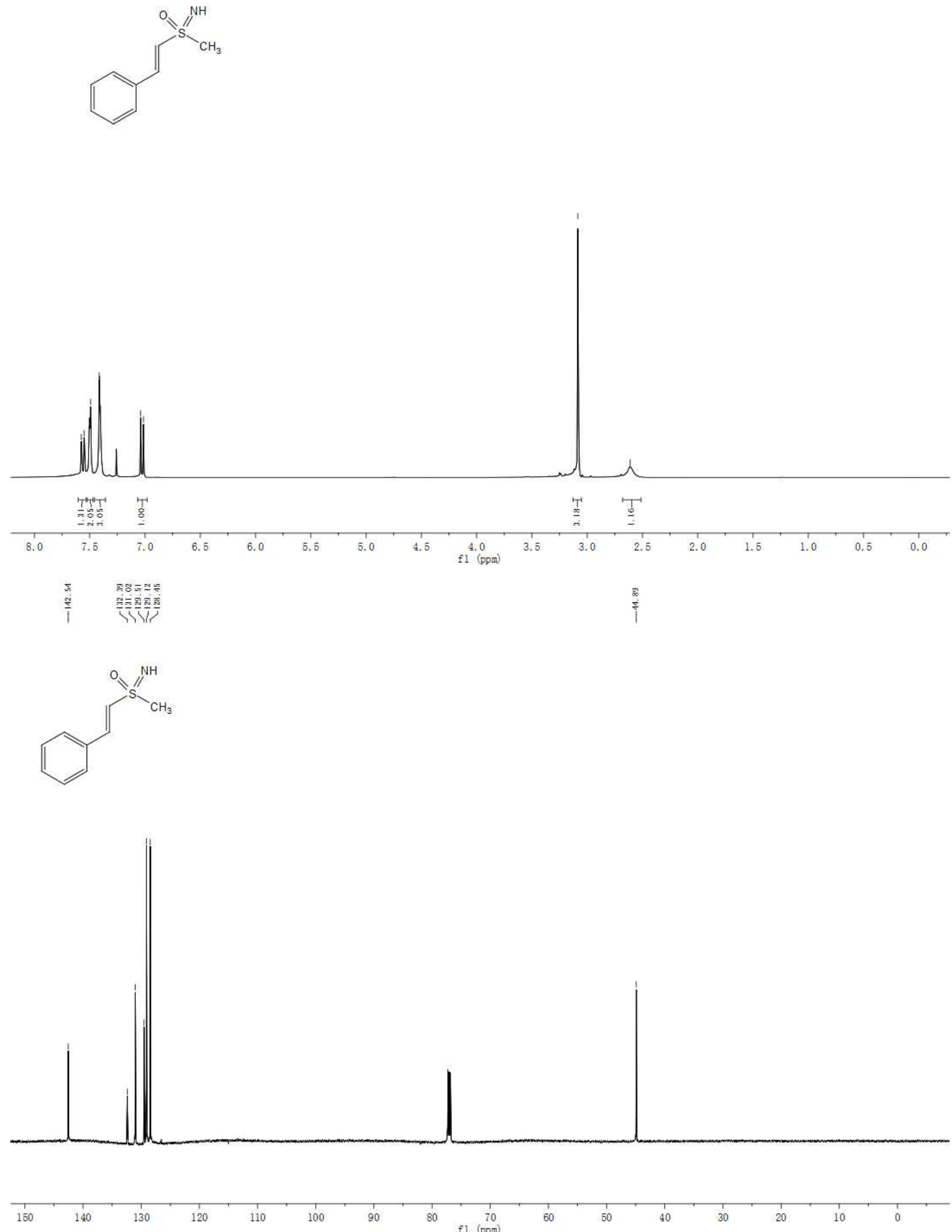